\title{
Structure of Tate-Shafarevich groups of elliptic curves over global function fields
}

\author{
M. L. Brown
}

\begin{abstract}
The structure of the Tate-Shafarevich groups of a class of elliptic curves over global function fields is determined. These are known to be finite abelian groups and hence they are direct sums of finite cyclic groups where the orders of these cyclic components are invariants of the Tate-Shafarevich group. This decomposition of the TateShafarevich groups into direct sums of finite cyclic groups depends on the behaviour of Drinfeld-Heegner points on these elliptic curves. These are points analogous to Heegner points on elliptic curves over the rational numbers.
\end{abstract}

\section{Contents}

Part 1. Preliminaries . . . . . . . . . . . . . . . . . 688

1.1. Introduction . . . . . . . . . . . . . . . . 688

1.2. Global fields of positive characteristic . . . . . . . . . . . . . . 690

1.3. Orders in imaginary quadratic field extensions . . . . . . . . . . . . 690

1.4. Ring class fields . . . . . . . . . . . . . . . . . . . 691

1.5. Elliptic curves over global fields of positive characteristic . . . . . . . 691

1.6. The Drinfeld modular curve $X_{0}^{\text {Drin }}(I) \ldots \ldots \ldots$. . . . . . . . 692

1.7. Analogue for $F$ of the Shimura-Taniyama-Weil conjecture . . . . . . 692

1.8. Drinfeld-Heegner points . . . . . . . . . . . . . . . . 692

1.9. Groups and cohomology . . . . . . . . . . . . . . . 693

1.10. Torsion on elliptic curves $E / F \ldots \ldots \ldots$. . . . . . . . . 693

1.11. Igusa's theorem . . . . . . . . . . . . . . . . . . . . 694

1.12. Consequences of Igusa's theorem . . . . . . . . . . . . . . . . . . 696

Part 2. Local duality, Cassels pairings, and Tate-Shafarevich groups . . . . . 697

2.1. Local duality of elliptic curves . . . . . . . . . . . . . . . . . . . 697

2.2. Selmer groups and Tate-Shafarevich groups . . . . . . . . . . . . . . . . 699

2.3. The Cassels pairing . . . . . . . . . . . . . . . . . . 700

Part 3. The cohomology classes $\gamma_{n}(c), \delta_{n}(c) \ldots \ldots \ldots \ldots$

3.1. The set $\mathcal{P}$ of prime numbers . . . . . . . . . . . . . . . 703

3.2. Frobenius elements and the set $\Lambda(n)$ of divisors . . . . . . . . . . 706

3.3. A refined Hasse principle for finite group schemes . . . . . . . . . . . 710

3.4. Drinfeld-Heegner points and the cohomology classes $\gamma_{n}(c), \delta_{n}(c) \ldots 714$

Part 4. Structure of the Tate-Shafarevich group and the Selmer group . . . . 720

4.1. Statement of the main theorems . . . . . . . . . . . . . . 720

4.2. Cochains for the cohomology classes $\gamma_{n}(c), \delta_{n}(c) \ldots \ldots \ldots 726$

4.3. Points $P_{c}$ defined over local fields . . . . . . . . . . . 728

4.4. The map $\chi_{z} \ldots \ldots \ldots \ldots \ldots$. . . . . . . . . . . . . . 729

Kyoto Journal of Mathematics, Vol. 55, No. 4 (2015), 687-772

DOI 10.1215/21562261-3157730, (C) 2015 by Kyoto University

Received December 6, 2012. Revised June 25, 2014. Accepted September 8, 2014.

2010 Mathematics Subject Classification: 11G05, 11G09, 11G20, 11G40, 14G10, 14G17, 14G25, $14 \mathrm{H} 52$. 
4.5. Localizations of the classes $\gamma_{n}(c)$ and $\delta_{n}(c) \ldots \ldots \ldots$. . . . . 740

4.6. The Cassels pairing with a class $\delta_{n}(c) \ldots \ldots \ldots \ldots 74$

Part 5. Construction of cohomology classes and proofs of the main theorems 744

5.1. $M_{r}$ is finite for some $r \ldots \ldots \ldots \ldots$. . . . . . . . . . . . . . . . . . . 744

5.2. A class $\gamma_{n}(c)$ in the Selmer group . . . . . . . . . . . . . . . . . 749

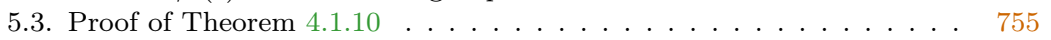

5.4. Proofs of Theorems 4.1 .4 and $4.1 .8 \ldots \ldots \ldots \ldots . \ldots \ldots 67 \ldots \ldots$

5.5. Proofs of Theorems 1.1 .1 and $4.1 .9 \ldots \ldots \ldots \ldots 765$

5.6. Generators of Tate-Shafarevich groups . . . . . . . . . . . . . . . 766

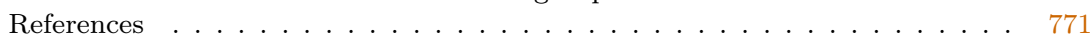

\section{Part 1. Preliminaries}

\subsection{Introduction}

Let $F$ be a global field of positive characteristic $p>0$. Let $E / F$ be an elliptic curve with an origin, that is to say, a 1-dimensional abelian variety.

In [1] it is shown that, for a class of these elliptic curves $E / F$, the TateShafarevich group $\amalg(E / F)$ is finite and, for prime numbers $l$ belonging to a set $\mathcal{S}$ of prime numbers given by arithmetic conditions, the l-primary component $\amalg(E / F)_{l_{\infty}}$ has order which is explicitly bounded.

In this paper, we determine the structure of the finite abelian group $\amalg(E / F)_{l^{\infty}}$ for the same class of elliptic curves and for all $l$ in the same set of prime numbers $\mathcal{S}$. (In the notation of Theorem 4.1.4 below, $\mathcal{S}$ is the set $\mathcal{P}$ with the exclusion of the prime divisors of the order of the Picard group $\operatorname{Pic}(A)$.) We also determine the structure of the Selmer groups of the elliptic curves in question.

Let $E / F$ be an elliptic curve, and let $K$ be an imaginary quadratic extension of $F$ with respect to the place $\infty$ of $F$ (see $\operatorname{Section} 1.2$ ). Let $\operatorname{Spec} A$ be the nonsingular affine curve with function field $F$ and whose point at infinity is $\infty$ (see Section 1.2). Assume that E, K, $\infty$ satisfy (a), (b), and (c) of Section 4.1.1. This provides an infinite set of prime numbers $\mathcal{P}$ of positive Dirichlet density and defined by arithmetic conditions (see Section 3.1). Indeed, $\mathcal{P}$ contains all except finitely many prime numbers $l \in \mathbb{Z}$ of the form $2^{s} n+1$ where $s \geq 1$ and $n$ is odd such that $q$ is a $2^{s}$ th-power nonresidue modulo $l$ where $q$ is the order of the exact finite field of constants of $F$.

Fix a prime number $l \in \mathcal{P}$. There are sets of divisors $\Lambda^{r}(n)$, relative to $l$, on $F$ for all integers $n \geq 1$ such that each divisor in $\Lambda^{r}(n)$ is a sum of $r$ distinct prime divisors and there is a decreasing filtration on $\Lambda^{r}(1)$ :

$$
\Lambda^{r}=\Lambda^{r}(1) \supseteq \Lambda^{r}(2) \supseteq \cdots .
$$

For any divisor $c \in \Lambda^{r}(n)$ there is a corresponding Drinfeld-Heegner point $P_{c}$ of $E(K[c])$, the group of $K[c]$-rational points of $E$, where $K[c]$ is the ring class field of $K$ with conductor $c$ (see Sections 1.4, 3.4.8).

On $E(K[c])$ there is the decreasing $l$-adic filtration

$$
E(K[c]) \supseteq l E(K[c]) \supseteq l^{2} E(K[c]) \supseteq \cdots .
$$


Define

$$
M_{r}=\min _{c \in \Lambda^{r}}\left(\max \left(n \in \mathbb{N} \mid P_{c} \in l^{n} E(K[c])\right)\right) \quad \text { for all integers } r \in \mathbb{N} .
$$

If the point $P_{0} \in E(K)$ has infinite order in the group of $K$-rational points $E(K)$, then it can be shown that $M_{0}, M_{1}, \ldots$ is a decreasing sequence of nonnegative integers (see Lemma 5.1.2). One of the main results of this paper is the following.

\section{THEOREM 1.1.1}

Suppose that $P_{0}$ has infinite order in $E(K)$, the group of $K$-rational points of $E$. Let $l$ be a prime number in $\mathcal{P}$ that is coprime to the order of the Picard group of the affine curve $\operatorname{Spec} A$. Let $\epsilon= \pm 1$ be the sign in the functional equation of the $L$-function of $E / F$. Then the Tate-Shafarevich group $\amalg(E / F)$ of $E / F$ is finite and its l-primary component is given by

$$
\amalg(E / F)_{l^{\infty}} \cong \prod_{\substack{(-1)^{i}=\epsilon \\ i \geq 0}}\left(\mathbb{Z} / l^{M_{i}-M_{i+1}} \mathbb{Z}\right)^{2},
$$

where the product runs over integers $i \in \mathbb{N}$ such that $(-1)^{i}=\epsilon$.

A similar statement holds for the Tate-Shafarevich group of the elliptic curve $E \times_{F} K$ over $K$ (see Theorem 4.1.4) as well as the Selmer groups of these curves (see Corollary 4.1.11). The main results of this paper are stated in Section 4.1.

It may be conjectured that for every global field $F$ of positive characteristic there are infinitely many nonisomorphic elliptic curves $E / F$ and infinitely many imaginary quadratic field extensions $K / F$ such that $E, K, \infty$ satisfy the hypotheses of Theorem 1.1.1 and those of Section 4.1. If this conjecture holds, then the above theorem and those of Section 4.1 give infinitely many nonisomorphic elliptic curves over a given global field of positive characteristic whose $l$-primary components of the Tate-Shafarevich group are structurally known for infinitely many prime numbers $l$ satisfying arithmetic conditions.

The method of this paper is related to that of Kolyvagin's determination of the structure of Tate-Shafarevich groups of a class of elliptic curves over the rational numbers (see [2], [3], [5]-[9]). The proofs of the main theorems of this paper stated in Section 4.1 and Theorem 1.1.1 above require many preliminary results which are explained in Parts $1-5$.

Part 2 contains basics on Tate local duality, Selmer groups, and the Cassels pairing on Tate-Shafarevich groups. In Section 3.1, the set $\mathcal{P}$ of prime numbers is defined by arithmetic conditions. In Section 3.2, the sets $\Lambda^{r}(n)$ of divisors on the global field $F$ are defined. Sections 3.3 and 3.4 construct the cohomology classes $\gamma_{n}(c), \delta_{n}(c)$ in the cohomology of the elliptic curve $E / F$.

In Section 4.1, the main results of this paper are stated, which are then proved in Sections 5.3-5.5 after some further properties of $\gamma_{n}(c), \delta_{n}(c)$ are proved in Parts 4 and 5 . We show, in particular, that the cohomology classes $\delta_{n}(c)$ define characters via the Cassels pairing on $\amalg(E / F)_{l}$, which determine the structure 
of this group. The method of proof of the main results in Section 4.1 is by the construction of many independent elements of the Tate-Shafarevich group $\amalg(E / F)_{l \infty}$. Finally, Section 5.6 contains complements to the main results.

While care has been taken to minimize the number of hypotheses required for the main theorems of this paper, these hypotheses are still numerous (see, e.g., Section 3.1, Definition 3.1.2). The assiduous reader will have an abundance of interesting problems in their elimination.

\subsection{Global fields of positive characteristic}

The notation of this paper is mainly that of [1] and is detailed in the rest of this section. A few differences arise, notably the sets of divisors $\Lambda(n)$, which are required for the more refined results of this paper.

Let

$k$ be a finite field of characteristic $p$ with $q=p^{m}$ elements;

$\bar{k}$ be an algebraic closure of $k$;

$C / k$ be a smooth projective irreducible curve over $k$;

$F$ be the function field of $C$. These hypotheses imply that the finite field $k$ is the exact field of constants of the global field $F$. Furthermore, let

$\Sigma_{L}$, for any global field $L$, be the set of all places of the field $L$;

$\infty \in \Sigma_{F}$ be a closed point of $C / k$;

$\kappa(z)$ be the residue field at a place $z \in \Sigma_{F}$ of $F$;

$F_{v}$ be the completion of $F$ at the place $v \in \Sigma_{F}$;

$F^{\text {sep }}$ be the separable closure of $F$;

$A$ be the coordinate ring $\Gamma\left(C \backslash\{\infty\}, \mathcal{O}_{C}\right)$ of the affine curve $C \backslash\{\infty\}$;

$\operatorname{Div}_{+}(A)$ be the semigroup of effective $k$-rational divisors on $\operatorname{Spec} A$. That is to say, $\operatorname{Div}_{+}(A)$ is the semigroup of effective $k$-rational divisors on $C / k$ which are coprime to the place $\infty$; an element of $\operatorname{Div}_{+}(A)$ may be written as a finite linear combination $\sum_{i} n_{i} z_{i}$ where $n_{i} \in \mathbb{N}$ and $z_{i}$ are prime divisors on $\operatorname{Spec} A$ for all $i$. Let

$\operatorname{Supp}(c)$, for a divisor $c \in \operatorname{Div}_{+}(A)$, be the support of the divisor $c$ which is the set of prime divisors with nonzero coefficient in $c$;

$\operatorname{Pic}(A)$ be the Picard group of $A$, the group of projective $A$-modules of rank 1;

$K$ be a separable imaginary quadratic extension field of $F$ with respect to $\infty$ (That is to say, $K$ is a quadratic extension field of $F$ in which the place $\infty$ remains inert.);

$B$ be the integral closure of $A$ in $K$;

$\tau$ be the nontrivial element of the Galois group $\operatorname{Gal}(K / F)$.

\subsection{Orders in imaginary quadratic field extensions}

Let $K / F$ be the imaginary quadratic field extension with respect to $\infty$ of Section 1.2. 
An order $O$ in $K$ with respect to $A$ is an $A$-subalgebra of $B$ whose fraction field is equal to $K$.

There is a bijection between orders $O_{c}$ of $K$ with respect to $A$ and effective $k$-rational divisors $c$ in $\operatorname{Div}_{+}(A)$ and it is given by

$$
c \mapsto A+B I(c),
$$

where $I(c)$ is the ideal of $A$ cutting out the divisor $c$. The divisor $c$ is the conductor of the order $O_{c}$. For more details on orders in imaginary quadratic extensions, see [1, Section 2.2].

\subsection{Ring class fields}

Let

$O_{c}$ be the order of $K$ with respect to $A$ and with conductor $c$ where $c \in \operatorname{Div}_{+}(A)$ (see Section 1.3);

$A_{v}$, for each place $v$ of $A$, be the localization of $A$ at $v$;

$\widehat{O}_{c, v}$ be the completion of the semilocal ring $O_{c} \otimes_{A} A_{v}$;

$G_{c}=K_{\infty}^{*} \prod_{v} \widehat{O}_{c, v}^{*}$ be the subgroup of the idèle group of the global field $K$ whose components are the units of $\widehat{O}_{c, v}$ for all places $v \neq \infty$ of $F$ and $K_{\infty}^{*}$ for the place $v=\infty$ and where in the product $v$ runs over all places of $F$;

$K[c]$, for any divisor $c \in \operatorname{Div}_{+}(A)$, be the ring class field with conductor $c$ with respect to $\infty$ (This is the finite abelian extension field of $K$ defined by the subgroup $G_{c}$ of the idèle group of $K$ via the reciprocity map.); and

$G\left(c / c^{\prime}\right)$ be the Galois group of the field extension $K[c] / K\left[c^{\prime}\right]$ for divisors $c \geq c^{\prime}$ of $\operatorname{Div}_{+}(A)$.

We have the following properties of the decomposition of primes in ring class fields (for the proofs, see [1, Section 2.3.13])

(a) The primes ramified in $K[c] / K$ are precisely the primes in the support of $c$.

(b) The extension $K[c] / K$ is split completely at the place of $K$ lying above $\infty$.

(c) If $z \notin \operatorname{Supp}(c)$, then for any positive integer $n$, the Galois extension $K[c+$ $n z] / K[c]$ is totally ramified at all places of $K[c]$ above $z$.

See [1, Section 2.3] for more details on ring class fields.

\subsection{Elliptic curves over global fields of positive characteristic}

Let

$C / k$ be a smooth projective irreducible curve over $k$ (as in Section 1.2);

$X / k$ be an elliptic surface over $C$ (That is to say, $X / k$ is a smooth projective irreducible surface equipped with a morphism $f: X \rightarrow C$ which has a section such that all fibers of $f$, except a finite number, are elliptic curves.); 
$E / F$ be the generic fiber of $f: X \rightarrow C$, which is an elliptic curve $E$ over $F$ equipped with an origin where $F$ is the function field of $C$.

The conductor of an elliptic curve $E / F$ is an effective $k$-rational divisor on $F$ supported only at the places of bad reduction of $E$ and whose multiplicities are defined in terms of the Galois representation of $\operatorname{Gal}\left(F^{\mathrm{sep}} / F\right)$ given by $E$. (See [1, Sections B.11.1-B.11.4] for the definition of the conductor of $E / F$.)

\subsection{The Drinfeld modular curve $X_{0}^{\operatorname{Drin}}(I)$}

Let $I$ be a nonzero ideal of $A$, and let $X_{0}^{\text {Drin }}(I)$ be the curve which is the coarse moduli scheme of Drinfeld modules of rank 2 for $A$ equipped with an $I$-cyclic structure (see [1, Definition 2.4.2, p. 23]). This curve is compactified by a finite number of cusps which correspond to "degenerate" Drinfeld modules. This curve $X_{0}^{\text {Drin }}(I)$ is an analogue for the global field $F$ of the classical modular curve $X_{0}(N)$, which is the coarse moduli scheme of elliptic curves equipped with a cyclic subgroup of order $N$, where $N \in \mathbb{N}$ (for more details, see [1, Section 2.4]).

\subsection{Analogue for $F$ of the Shimura-Taniyama-Weil conjecture}

Let $E / F$ be an elliptic curve with split (Tate) multiplicative reduction at $\infty$. Let $I$ be the nonzero ideal of the ring $A$ which is the conductor, without the place at $\infty$, of the elliptic curve $E / F$.

According to the work of Drinfeld on the Langlands conjecture, there is a finite surjective morphism of curves over $F$

$$
X_{0}^{\operatorname{Drin}}(I) \rightarrow E \text {. }
$$

This result is an analogue for the global field $F$ of the Shimura-TaniyamaWeil conjecture proved by Wiles for semistable elliptic curves over the rational numbers.

For more details, see [1, Section 4.7, Appendix B].

\subsection{Drinfeld-Heegner points}

Let $K$ be an imaginary quadratic extension field of $F$ with respect to $\infty$ (as in Section 1.2), and let $I$ be a nonzero ideal of $A$.

Let $D$ be a rank 2 Drinfeld module for $A$ with complex multiplication by an order $\mathcal{O}$ of the field $K$ with respect to $A$; that is to say, $\mathcal{O}$ is a subring of $K$ which is integral over $A$. Let $Z$ be an $I$-cyclic subgroup of $D$. Then the pair $(D, Z)$ represents a noncuspidal point of the modular curve $X_{0}^{\text {Drin }}(I)$. Such points $(D, Z)$ exist if the prime divisors in the support of $I$ split completely in the field extension $K / F$.

If the quotient Drinfeld module $D / Z$ has the same ring of endomorphisms $\mathcal{O}$ as $D$, then the point $(D, Z)$ on $X_{0}^{\text {Drin }}(I)$ is called a Drinfeld-Heegner point.

If $f: X_{0}^{\operatorname{Drin}}(I) \rightarrow E$ is a finite morphism of curves where $E / F$ is an elliptic curve (see Sections 1.5-1.7), then the point $f(D, Z)$ of the elliptic curve $E$ is also called a Drinfeld-Heegner point. 
The Drinfeld-Heegner points $(D, Z)$ and $f(D, Z)$ are rational over the ring class field $K[c]$ where $c$ is the conductor of the order $\mathcal{O}$ of $K$ relative to $A$.

See [1, Sections 2.2, 2.3] or Section 3.4 below for more details.

\subsection{Groups and cohomology}

If $G$ is a discrete abelian group, denote by

$G_{m}$ the kernel of multiplication by the integer $m \in \mathbb{N}$ on $G$;

${ }_{m} G$ the cokernel $G / m G$ of multiplication by the integer $m \in \mathbb{N}$;

$|G|$ the order of the group $G$, which is either a positive integer or $+\infty$;

$\operatorname{ord}(g)$ the order of an element $g \in G$, which is the cardinality of the subgroup generated by $g$;

$\exp (G)$ the exponent of $G$, which is the maximum order of an element of $G$;

$\widehat{G}$ the Pontryagin dual of $G$, namely, the topological group $\operatorname{Hom}(G, \mathbb{Q} / \mathbb{Z})$.

If $G$ is a finite abelian group, then $\widehat{G}$ may be identified with the group of 1-dimensional complex characters of $G$, that is to say, the group of homomorphisms $\operatorname{Hom}\left(G, \mathbb{C}^{*}\right)$. A character of a finite abelian group is always assumed to be irreducible.

If $F^{\prime} / F$ is a Galois extension of a global field $F$ and if $z \in \Sigma_{F}$ is a prime divisor of $F$ unramified in $F^{\prime}$, then we denote by $\operatorname{Frob}(z)$ or $\operatorname{Frob}_{F^{\prime} / F}(z)$, the conjugacy class in $\operatorname{Gal}\left(F^{\prime} / F\right)$ of Frobenius substitutions associated to $z$.

If $L$ is a field, then we write $H^{i}(L, M)$ for the Galois cohomology group $H^{i}\left(\operatorname{Gal}\left(L^{\mathrm{sep}} / L\right), M\right)$, where $L^{\text {sep }}$ is the separable closure of $L$. If $L^{\prime} / L$ is a finite Galois field extension, then we write $H^{i}\left(L^{\prime} / L, M\right)$ for $H^{i}\left(\operatorname{Gal}\left(L^{\prime} / L\right), M\right)$.

If $F$ is a global field and $z$ is a place of $F$, then the restriction, or localization, of a class $c \in H^{i}(L, M)$ is written $c_{z} \in H^{i}\left(F_{z}, M\right)$, where $F_{z}$ is the completion of $F$ at $z$.

\subsection{Torsion on elliptic curves $E / F$}

Let $E$ be an elliptic curve over a global field $F$ of positive characteristic $p>0$ as in Section 1.5. Let $K$ be an imaginary quadratic extension field of $F$ with respect to the place $\infty$ of $F$. As in Section 1.4, let $K[c]$ be the ring class field over $K$ with conductor $c \in \operatorname{Div}_{+}(A)$. Put

$$
K[A]=\bigcup_{c \in \operatorname{Div}_{+}(A)} K[c]
$$

That is to say, $K[A]$ is a field which is the union of all the ring class fields $K[c]$ in some algebraic closure of $K$.

Let $S$ be a subset of $\mathbb{N}^{*}$ such that if $n_{1} \in S$ and $n_{0}$ is any divisor of $n_{1}$, then $n_{0} \in S$. A quasigroup $\left\{G_{n}\right\}_{n \in S}$ relative to $S$ is a family of abelian groups $G_{n}$ indexed by the elements $n$ of $S$ such that $n G_{n}=0$ and if $n_{0}, n_{1} \in S$ are elements where $n_{0}$ divides $n_{1}$, then there is a group homomorphism $f_{n_{1} n_{0}}: G_{n_{1}} \rightarrow G_{n_{0}}$ 
satisfying the compatibility condition that if $n_{0}, n_{1}, n_{2} \in S, n_{0}$ divides $n_{1}$, and $n_{1}$ divides $n_{2}$, then $f_{n_{2} n_{0}}=f_{n_{1} n_{0}} \circ f_{n_{2} n_{1}}$ (see [1, Section 7.1, p. 330]).

PROPOSITION 1.10.1 ([1, PROPOSITION 7.3.8])

The quasigroup

$$
\left\{E(K[A])_{n}\right\}_{n \in \mathbb{N}}
$$

is trivial; that is to say, the order of the torsion group $E(K[A])_{n}$ is bounded independently of $n$ and there is a finite set $\mathcal{E}$ of prime numbers such that for all integers $n$ prime to all elements of $\mathcal{E}$ we have

$$
E(K[A])_{n} \cong 0
$$

PROPOSITION 1.10.2 ([1, PROPOSITION 7.14.2])

Let $\mathcal{E}$ be the finite set of prime numbers of Proposition 1.10.1. For any divisor $c$ of $\operatorname{Div}_{+}(A)$, the restriction homomorphism

$$
H^{1}\left(K, E_{n}\right) \rightarrow H^{1}\left(K[c], E_{n}\right)^{\mathcal{G}_{c}}
$$

is an isomorphism for all integers $n$ prime to $\mathcal{E}$ where $\mathcal{G}_{c}=\operatorname{Gal}(K[c] / K)$.

This follows from Proposition 1.10.1 and the Hochschild-Serre spectral sequence

$$
H^{i}\left(\mathcal{G}_{c}, H^{j}\left(K[c], E_{n}\left(K^{\mathrm{sep}}\right)\right)\right) \Rightarrow H^{i+j}\left(K, E_{n}\left(K^{\mathrm{sep}}\right)\right)
$$

(more details are given in [1, Proposition 7.14.2]).

\subsection{Igusa's theorem}

This section is a summary of the results of Igusa for the Galois action on torsion points of elliptic curves over global fields of positive characteristic.

\subsection{1.}

As in Section 1.2, let $F$ be a global field of positive characteristic $p$ where $k$ is the exact field of constants of $F$, and let $E / F$ be an elliptic curve. Let

$G=\operatorname{Gal}\left(F^{\mathrm{sep}} / F\right)$, where $F^{\text {sep }}$ is the separable closure of $F$;

$n$ be an integer prime to $p$;

$E_{n}$ be the finite $F$-group scheme of $n$-torsion points of $E$;

$E_{\infty}$ be the torsion subgroup of $E\left(F^{\text {sep }}\right)$ of order prime to $p$.

The elliptic curve $E / F$ is said to be isotrivial if there is a finite Galois extension field $F^{\prime}$ of $F$ such that the curve $E \times{ }_{F} F^{\prime}$ is definable over a finite subfield of $F^{\prime}$.

\subsection{2.}

The action of the Galois group $G$ on $E_{n}$ provides a homomorphism

$$
\rho_{n}: G \rightarrow \operatorname{Aut}\left(E_{n}\right) \cong \mathrm{GL}_{2}(\mathbb{Z} / n \mathbb{Z}) .
$$


The determinant

$$
\operatorname{det}: \operatorname{Aut}\left(E_{n}\right) \rightarrow(\mathbb{Z} / n \mathbb{Z})^{*}
$$

induces a homomorphism

$$
G \rightarrow(\mathbb{Z} / n \mathbb{Z})^{*}
$$

Let $H_{n}$ be the subgroup of $(\mathbb{Z} / n \mathbb{Z})^{*}$ generated by the powers of $q=|k|$ modulo $n$. Then $H_{n}$ is naturally isomorphic to the Galois group of the field of $n$th roots of unity over $k$. Let $\Gamma_{n}$ be the subgroup of $\mathrm{GL}_{2}(\mathbb{Z} / n \mathbb{Z})$ defined by the exact sequence of finite groups, where det is the restriction to $\Gamma_{n}$ of the determinant homomorphism on $\mathrm{GL}_{2}(\mathbb{Z} / n \mathbb{Z})$,

$$
0 \quad \rightarrow \quad \mathrm{SL}_{2}(\mathbb{Z} / n \mathbb{Z}) \quad \rightarrow \quad \Gamma_{n} \stackrel{\text { det }}{\rightarrow} H_{n} \quad \rightarrow \quad 0
$$

\subsection{3.}

Passing to the projective limit of the previous exact sequence over all integers $n$ prime to $p$, we obtain the exact sequence of profinite groups

$$
0 \longrightarrow \mathrm{SL}_{2}\left(\widehat{\mathbb{Z}}^{(p)}\right) \longrightarrow \widehat{\Gamma} \longrightarrow \widehat{H} \longrightarrow 0
$$

where $\widehat{H}$ is the subgroup of $\widehat{\mathbb{Z}}^{(p) *}$ topologically generated by $q$, where

$$
\widehat{\mathbb{Z}}^{(p)}=\prod_{l \neq p} \mathbb{Z}_{l}
$$

is the profinite prime-to- $p$ completion of $\mathbb{Z}$, and $\widehat{\Gamma}$ is a closed subgroup of $\mathrm{GL}_{2}\left(\widehat{\mathbb{Z}}^{(p)}\right)$.

1.11.4.

Passing to the projective limit of the exact sequence (1.11.1) where $n$ runs over all powers of a prime number $l$ where $l \neq p$, we obtain the exact sequence

$$
0 \longrightarrow \mathrm{SL}_{2}\left(\mathbb{Z}_{l}\right) \longrightarrow \widehat{\Gamma}_{l} \longrightarrow \widehat{H}_{l} \longrightarrow 0 \text {. }
$$

THEOREM 1.11.1 (IGUSA [4])

Suppose that $E / F$ is not isotrivial. Then the profinite group $\operatorname{Gal}\left(F\left(E_{\infty}\right) / F\right)$ is an open subgroup of $\widehat{\Gamma}$.

This result has the following consequence.

THEOREM 1.11.2

Suppose that $E / F$ is not isotrivial. Then for all prime numbers $l \neq p$ the profinite group $\operatorname{Gal}\left(F\left(E_{l}\right) / F\right)$ is an open subgroup of $\widehat{\Gamma}_{l}$ and is equal to $\widehat{\Gamma}_{l}$ for all but finitely many $l$.

REMARKS 1.11.3

(a) Suppose that the curve $E / F$ is isotrivial. Then it is easy to show that the 
group $\operatorname{Gal}\left(F\left(E_{\infty}\right) / F\right)$ is an extension of a finite group by the abelian profinite group $\widehat{\mathbb{Z}}^{(p)}$.

(b) Let $E$ be an elliptic curve defined over a number field $L$. The Galois action on the torsion points of $E / L$ is known and depends principally on whether or not $E$ has complex multiplication.

See [12] and [12, Section 4.5] for the cases of complex multiplication and without complex multiplication. See also [1, Remarks 7.2.8] for more details.

\subsection{Consequences of Igusa's theorem}

For a finite group $G$ and a $\mathbb{Z}[G]$-module $M$, let $H^{i}(G, M)$ denote the standard cohomology groups of $G$ acting on $M$ (see [1, Section 5.6]; see also [10, Chapter I] for the Tate modified cohomology groups).

PROPOSITION 1.12.1

Let $E / F$ be an elliptic curve, and let $\mathbb{N}^{(p)}$ be the set of positive integers coprime to $p$, where $p$ is the characteristic of $F$. Write $G_{n}$ for the group $\operatorname{Gal}\left(F\left(E_{n}\right) / F\right)$.

(a) Let $i=0$ or 1 . Then

$$
\left\{H^{i}\left(G_{n}, E_{n}\right)\right\}_{n \in \mathbb{N}(p)}
$$

is a trivial quasigroup.

(b) There is a finite set $\mathcal{N}$ of prime numbers including $p$ such that for all prime numbers $l \notin \mathcal{N}$ we have

$$
H^{i}\left(G_{l^{n}}, E_{l^{n}}\right)=0 \text { for all } n \geq 1 \text { and all } i \geq 0 .
$$

Proposition 1.12.1(a) may be restated as follows: for $i=0$ or 1 and for all $n$ coprime to $p$, the order of the group $H^{i}\left(G_{n}, E_{n}\right)$ is bounded independently of $n$ and there is a finite set of prime numbers such that for all integers $n$ coprime to this set of prime numbers we have $H^{i}\left(G_{n}, E_{n}\right) \cong 0$. For the proof of this proposition, see [1, Proposition 7.3.1].

\subsection{1.}

For each prime number $l$ different from $p$, select once and for all a basis of the Tate module $T_{l}(E)$ over $\mathbb{Z}_{l}$, the $l$-adic completion of $\mathbb{Z}$; this fixes for the rest of this paper, for every prime number $l \neq p$, an isomorphism of $\operatorname{Gal}\left(F\left(E_{l \infty}\right) / F\right)$ with a subgroup of $\mathrm{GL}_{2}\left(\mathbb{Z}_{l}\right)$ and the two groups may then be identified with each other.

PROPOSITION 1.12.2

Let $E / F$ be an elliptic curve which is not isotrivial. Let $L$ be a finite extension field of $F$ in which $k$ is algebraically closed. Then there is an infinite set $S$ of prime numbers of positive Dirichlet density such that for all $l \in S$ we have that 
(a) the fields $F\left(E_{l \infty}\right)$ and $L$ are linearly disjoint over $F$;

(b) $E(L)_{l \infty}=0$;

(c) $\left(\begin{array}{cc}1 & 0 \\ 0 & -1\end{array}\right) \in \operatorname{Gal}\left(F\left(E_{l \infty}\right) / F\right)$.

For the proof, see [1, Proposition 7.3.10].

\section{Part 2. Local duality, Cassels pairings, and Tate-Shafarevich groups}

\subsection{Local duality of elliptic curves}

This section is a brief summary of Tate-Poitou local duality for elliptic curves over a local field. For more details on local duality of abelian varieties, see [10, Chapter I; Chapter III, Section 7].

\subsection{1.}

Let

$L$ be a nonarchimedean complete local field;

$L^{\text {sep }}$ be the separable closure of $L$;

$E / L$ be an elliptic curve over $L$;

$n \geq 1$ be an integer coprime to the characteristic of $L$;

$G$ be the Galois group $\operatorname{Gal}\left(L^{\mathrm{sep}} / L\right)$.

\subsection{2.}

Let $\mu_{n}$ be the multiplicative subgroup of $L^{\text {sep }}$ of $n$th roots of unity. Then $\mu_{n}$ is a finite $G$-module. Let $E_{n}$ denote the $G$-module of $n$-torsion points of $E\left(L^{\text {sep }}\right)$. We have an abelian group isomorphism

$$
E_{n} \cong \frac{\mathbb{Z}}{n \mathbb{Z}} \oplus \frac{\mathbb{Z}}{n \mathbb{Z}}
$$

Denote by $\{\cdot, \cdot\}$ the Weil pairing

$$
\{\cdot, \cdot\}: E_{n} \times E_{n} \rightarrow \mu_{n} .
$$

This is a perfect pairing of $G$-modules. In particular, we have an isomorphism of $G$-modules

$$
E_{n} \cong \operatorname{Hom}_{G}\left(E_{n}, \mu_{n}\right) .
$$

\subsection{3.}

The Weil pairing induces a cup-product pairing in Galois cohomology

$$
H^{1}\left(L, E_{n}\right) \times H^{1}\left(L, E_{n}\right) \rightarrow H^{2}\left(L, \mu_{n}\right) .
$$

This is a nondegenerate antisymmetric pairing of abelian groups. By local class field theory, we have a canonical isomorphism of groups, where $\operatorname{Br}(L)$ is the Brauer group of $L$,

$$
\operatorname{Br}(L) \cong \frac{\mathbb{Q}}{\mathbb{Z}}
$$


This induces an isomorphism

$$
H^{2}\left(L, \mu_{n}\right)=\operatorname{Br}(L)_{n} \cong \frac{\mathbb{Z}}{n \mathbb{Z}}
$$

THEOREM 2.1.1 (TATE-POITOU LOCAL DUALITY)

The cup-product pairing

$$
\langle\cdot, \cdot\rangle_{v}: H^{1}\left(L, E_{n}\right) \times H^{1}\left(L, E_{n}\right) \rightarrow \frac{\mathbb{Z}}{n \mathbb{Z}}
$$

obtained from the Weil pairing is an alternating and nondegenerate pairing of $\mathbb{Z} / n \mathbb{Z}$-modules.

For the proof, see [10, Chapter I, Corollary 2.3].

\section{THEOREM 2.1.2}

Assume that $n$ is prime to the residue field characteristic of $L$.

(a) The subgroup ${ }_{n} E(L)$ of $H^{1}\left(L, E_{n}\right)$ is isotropic for the alternating pairing $\langle\cdot, \cdot\rangle_{v}$.

(b) The cup-product pairing $\langle\cdot, \cdot\rangle_{v}$ on $H^{1}\left(L, E_{n}\right)$ induces a nondegenerate pairing of abelian groups, where ${ }_{n} E(L)=E(L) / n E(L)$,

$$
[\cdot, \cdot]_{v}:{ }_{n} E(L) \times H^{1}(L, E)_{n} \rightarrow \frac{\mathbb{Z}}{n \mathbb{Z}} .
$$

For the proof, see [1, Theorem 7.15.6, p. 403] for part (a) and [10, Chapter I, Corollary 3.4 and Remark 3.6; Chapter 3, Theorem 7.8] for part (b). Note that part (b) holds without the hypothesis that $n$ be coprime to the characteristic of $L$ (see [10, Chapter III, Section 7]).

\subsection{4.}

Suppose now that $K$ is a global field of positive characteristic, that $\Sigma_{K}$ is the set of all places of $K$, and that the integer $n$ is coprime to the characteristic of $K$. Let $E / K$ be an elliptic curve.

\section{PROPOSITION 2.1.3}

Let $c$ and $c^{\prime}$ be two elements of $H^{1}\left(K, E_{n}\right)$. Denote by $c_{v}$ and $c_{v}^{\prime}$ the induced elements of $H^{1}\left(K_{v}, E_{n}\right)$ for all places $v \in \Sigma_{K}$ of $K$, where $K_{v}$ is the completion of $K$ at $v$. Then we have

$$
\sum_{v \in \Sigma_{K}}\left\langle c_{v}, c_{v}^{\prime}\right\rangle_{v}=0
$$

Proof

The sum of the local invariants of a global class in $H^{2}\left(K, \mathbb{G}_{m}\right)$ is zero. 


\subsection{Selmer groups and Tate-Shafarevich groups}

\subsection{1.}

Let $E / F$ be an elliptic curve as in Section 1.5, and let $n \in \mathbb{N}$ be an integer coprime to the characteristic $p$ of the global field $F$.

\subsection{2.}

For a place $v$ of the field $F$, we write $F_{v}$ for the completion of $F$ at the place $v$ (as in Section 1.2). The exact sequence of commutative group schemes over $F$, obtained from the morphism of multiplication by $n$,

$$
0 \longrightarrow E_{n} \longrightarrow E \stackrel{n}{\longrightarrow} E \longrightarrow 0
$$

gives rise to a commutative diagram, where the maps $\operatorname{res}_{v}$ are the restriction homomorphisms at $v$ and the rows are exact sequences of abelian groups:

$$
\begin{aligned}
& 0 \rightarrow{ }_{n} E(F) \rightarrow H^{1}\left(F, E\left(F^{\mathrm{sep}}\right)_{n}\right) \rightarrow H^{1}\left(F, E\left(F^{\mathrm{sep}}\right)\right)_{n} \quad \rightarrow \quad 0 \\
& \downarrow \operatorname{res}_{v} \quad \downarrow \operatorname{res}_{v} \quad \downarrow \operatorname{res}_{v} \\
& 0 \rightarrow{ }_{n} E\left(F_{v}\right) \rightarrow H^{1}\left(F_{v}, E\left(F_{v}^{\mathrm{sep}}\right)_{n}\right) \rightarrow H^{1}\left(F_{v}, E\left(F_{v}^{\mathrm{sep}}\right)\right)_{n} \rightarrow 0
\end{aligned}
$$

As in Section 1.9, ${ }_{n} E(F)$ denotes the cokernel $E(F) / n E(F)$ and $E\left(F^{\mathrm{sep}}\right)_{n}$ denotes the $n$-torsion subgroup of $E\left(F^{\text {sep }}\right)$.

\subsection{3.}

The Tate-Shafarevich group $\amalg(E / F)$ of $E / F$ is defined as

$$
\amalg(E / F)=\operatorname{ker}\left\{H^{1}(F, E) \rightarrow \prod_{v \in \Sigma_{F}} H^{1}\left(F_{v}, E\right)\right\} .
$$

This group $\amalg(E / F)$ is known to be a torsion of cofinite type (see [11]).

The $n$-Selmer group is defined as

$$
\operatorname{Sel}_{n}(E / F)=\bigcap_{v \in \Sigma_{F}} \operatorname{res}_{v}^{-1}\left({ }_{n} E\left(F_{v}\right)\right)
$$

in terms of the commutative diagram of Section 2.2.2 and where res $v$ denotes the middle vertical homomorphism of the diagram. Therefore, $\operatorname{Sel}_{n}(E / F)$ is a subgroup of $H^{1}\left(F, E\left(F^{\mathrm{sep}}\right)_{n}\right)$ and is a finite abelian group. We then have the exact sequence of torsion abelian groups from the commutative diagram of Section 2.2.2, where $\amalg(E / F)_{n}$ is the $n$-torsion subgroup of $\amalg(E / F)$,

$$
0 \rightarrow{ }_{n} E(F) \rightarrow \operatorname{Sel}_{n}(E / F) \rightarrow \amalg(E / F)_{n} \rightarrow 0 .
$$

\subsection{4.}

Let $F^{\prime} / F$ be a finite separable Galois field extension. We write $\amalg\left(E / F^{\prime}\right)$ in place of $\amalg\left(E \times_{F} F^{\prime} / F^{\prime}\right)$ for the Tate-Shafarevich group of $E \times_{F} F^{\prime}$ over $F^{\prime}$ obtained by ground field extension from $F$ to $F^{\prime}$; similarly, for the Selmer quasigroup, we write $\operatorname{Sel}_{n}\left(E / F^{\prime}\right)$ in place of $\operatorname{Sel}_{n}\left(E \times_{F} F^{\prime} / F^{\prime}\right)$. 
PROPOSITION 2.2.1

Let $r$ be the degree of the finite separable field extension $F^{\prime} / F$. For any torsion abelian group $\mathcal{A}$ write $\mathcal{A}_{(\text {non-r) }}$ for the torsion subgroup of $\mathcal{A}$ of order coprime to $r$. The restriction homomorphism provides isomorphisms for all integers $n$ coprime to $r$

$$
\begin{gathered}
\amalg(E / F)_{(\text {non-r })} \stackrel{\stackrel{\text { res }}{\cong}}{\longrightarrow} \amalg\left(E / F^{\prime}\right)_{(\text {non-r })} \operatorname{Gal}\left(F^{\prime} / F\right), \\
\operatorname{Sel}_{n}(E / F) \stackrel{\stackrel{\text { res }}{\cong}}{\longrightarrow} \operatorname{Sel}_{n}\left(E / F^{\prime}\right)^{\operatorname{Gal}\left(F^{\prime} / F\right) .}
\end{gathered}
$$

Proof

The definition of the Tate-Shafarevich groups provides a commutative diagram with exact rows:

$$
\begin{aligned}
& 0 \rightarrow \amalg\left(E / F^{\prime}\right)^{\operatorname{Gal}\left(F^{\prime} / F\right)} \rightarrow H^{1}\left(F^{\prime}, E\right)^{\operatorname{Gal}\left(F^{\prime} / F\right)} \rightarrow\left(\prod_{v \in \Sigma_{F^{\prime}}} H^{1}\left(F_{v}^{\prime}, E\right)\right)^{\operatorname{Gal}\left(F^{\prime} / F\right)} \\
& \uparrow \quad \uparrow \quad \uparrow \\
& 0 \rightarrow \quad \amalg(E / F) \quad \rightarrow \quad H^{1}(F, E) \quad \rightarrow \quad \prod_{v \in \Sigma_{F}} H^{1}\left(F_{v}, E\right)
\end{aligned}
$$

For any place $v$ of $F$, the $F_{v}$-algebra $F_{v} \otimes_{F} F^{\prime}$ is étale and is the product of the completions of $F^{\prime}$ at the places lying over $v$. The inflation restriction sequence provides isomorphisms for any integer $s$ coprime to the order of $\operatorname{Gal}\left(F^{\prime} / F\right)$

$$
\begin{aligned}
& H^{1}(F, E)_{s} \cong H^{1}\left(F^{\prime}, E\right)_{s}^{\operatorname{Gal}\left(F^{\prime} / F\right)}, \\
& H^{1}\left(F_{v}, E\right)_{s} \cong H^{1}\left(F_{v} \otimes_{F} F^{\prime}, E\right)_{s}^{\operatorname{Gal}\left(F^{\prime} / F\right)} \text { for all places } v \text { of } F .
\end{aligned}
$$

The isomorphism of the proposition for the Tate-Shafarevich groups now follows by a diagram chase. The corresponding isomorphism for the $n$-Selmer groups follows from the commutative diagram with exact rows:

$$
\begin{aligned}
& \begin{array}{ccccc}
{ }_{n} E(F) & \rightarrow & \operatorname{Sel}_{n}(E / F) & \rightarrow & \amalg(E / F)_{n} \\
\downarrow & & \downarrow & & \downarrow 0
\end{array} \\
& 0 \rightarrow\left({ }_{n} E\left(F^{\prime}\right)\right)^{\operatorname{Gal}\left(F^{\prime} / F\right)} \rightarrow \operatorname{Sel}_{n}\left(E / F^{\prime}\right)^{\operatorname{Gal}\left(F^{\prime} / F\right)} \rightarrow \amalg\left(E / F^{\prime}\right)_{n}^{\operatorname{Gal}\left(F^{\prime} / F\right)} \rightarrow 0
\end{aligned}
$$

as required.

REMARK 2.2.2

This section is a generalized form of [1, Section 7.9].

\subsection{The Cassels pairing}

2.3.1.

Let $E / F$ be an elliptic curve over the global field $F$ of characteristic $p>0$ as in Section 1.5. The Tate-Shafarevich group $\amalg(E / F)$ of $E / F$ is equipped with the 
antisymmetric Cassels pairing

$$
\langle\cdot, \cdot\rangle_{\text {Cassels }}: \amalg(E / F) \times \amalg(E / F) \rightarrow \mathbb{Q} / \mathbb{Z},
$$

which is nondegenerate if $\amalg(E / F)$ is finite. The Tate-Shafarevich group $\amalg(E / F)$ is a torsion group of cofinite type (see [11]).

In this section, the Cassels pairing is defined for $E / F$ for the non- $p$ part $\amalg(E / F)_{(\text {non- } p)}$ of $\amalg(E / F)$, that is to say, the subgroup of the Tate-Shafarevich group of order coprime to the characteristic $p$.

\subsection{2.}

For any place $v \in \Sigma_{F}$ of $F$, we have the commutative diagram with exact rows obtained from restriction from $F$ to $F_{v}$ for any integer $m$ where $\partial_{m}$ is the connecting homomorphism induced from the morphism of multiplication by $m$ on $E$ :

$$
\begin{aligned}
& 0 \rightarrow E(F)_{m} \rightarrow E(F) \rightarrow E(F) \stackrel{\partial_{m}}{\rightarrow} H^{1}\left(F, E_{m}\right) \rightarrow H^{1}(F, E)_{m} \\
& 0 \rightarrow E\left(F_{v}\right)_{m} \rightarrow E\left(F_{v}\right) \rightarrow E\left(F_{v}\right) \stackrel{\partial_{m}}{\rightarrow} H^{1}\left(F_{v}, E_{m}\right) \rightarrow H^{1}\left(F_{v}, E\right)_{m}
\end{aligned}
$$

\subsection{3.}

Let $a, b \in \amalg(E / F)_{(\text {non- } p)}$. Let $m \geq 1$ be the order of $a$, and let $n \geq 1$ be the order of $b$ where $m, n$ are coprime to the characteristic $p$ of $F$. Then we have that

$$
a \in \amalg(E / F)_{m} \quad \text { and } \quad b \in \amalg(E / F)_{n} .
$$

Select elements

$$
a^{(1)} \in H^{1}\left(F, E_{m}\right) \quad \text { and } \quad b^{(1)} \in H^{1}\left(F, E_{n}\right)
$$

mapping to $a$ and $b$, respectively, in the commutative diagram of Section 2.3.2.

For any element $h \in H^{1}(F, E)$ denote by $h_{v}$ the restriction of $h$ to $H^{1}\left(F_{v}, E\right)$ for any place $v$ of $F$ and similarly for cochains.

\subsection{4.}

Suppose first that $a$ is divisible by $n$ in $H^{1}(F, E)$, say, $a=n a_{1}$ where $a_{1} \in$ $H^{1}(F, E)_{m n}$. We may select local points $y_{v} \in{ }_{n} E\left(F_{v}\right)$ such that

$$
\partial_{n}\left(y_{v}\right)=b_{v}^{(1)} \text { for all places } v \in \Sigma_{F},
$$

as $b_{v}^{(1)}$ maps to zero in $H^{1}\left(F_{v}, E\right)$. Let $a_{1, v}$ denote the localization in $H^{1}\left(F_{v}, E\right)_{m n}$ of $a_{1}$. Note that since $a \in \amalg(E / F)_{m}$ we have that $a_{1, v} \in H^{1}\left(F_{v}, E\right)_{n}$ for all $v$. For any $v \in \Sigma_{F}$, denote by

$$
[\cdot, \cdot]_{v}: H^{1}\left(F_{v}, E\right)_{n} \times{ }_{n} E\left(F_{v}\right) \rightarrow \frac{\mathbb{Z}}{n \mathbb{Z}}
$$

the local pairing as in Theorem 2.1.2. Then the Cassels pairing is given in terms of the local pairing by the formula 


$$
\langle a, b\rangle_{\text {Cassels }}=\sum_{v \in \Sigma_{F}}\left[a_{1, v}, y_{v}\right]_{v}
$$

where the sum runs over all places of $F$.

\subsection{5.}

We have that

$$
a_{1, v}=i_{*}\left(c_{1, v}\right)
$$

for some $c_{1, v} \in H^{1}\left(F_{v}, E_{n}\right)$ for all $v$ where $i$ is the inclusion of group schemes $E_{n} \hookrightarrow E$. We then have that the Cassels pairing is also given in terms of the cup-product pairing $\langle\cdot, \cdot\rangle_{v}$ of Theorem 2.1 .1 by

$$
\langle a, b\rangle_{\text {Cassels }}=\sum_{v}\left\langle c_{1, v}, b_{v}^{(1)}\right\rangle_{v}
$$

as we have for all places $v$

$$
\left[a_{1, v}, y_{v}\right]_{v}=\left\langle c_{1, v}, \partial_{n}\left(y_{v}\right)\right\rangle_{v}
$$

\subsection{6.}

In Sections 2.3.4 and 2.3.5, the global element $a_{1} \in H^{1}(F, E)_{m n}$ such that $n a_{1}=a$ may not exist. Nevertheless, in a suitable sense it always exists locally, and in general, the Cassels pairing is defined as follows.

Select elements $a^{(1)}$ and $b^{(1)}$ of $H^{1}\left(F, E_{m}\right)$ and $H^{1}\left(F, E_{n}\right)$ mapping to $a$ and $b$, respectively. For each valuation $v$ of $F$, let $a_{v}^{(1)}$ be the localization in $H^{1}\left(F_{v}, E_{m}\right)$ of $a^{(1)} \in H^{1}\left(F, E_{m}\right)$. For each valuation $v$ of $F$, $a$ maps to zero in $H^{1}\left(F_{v}, E\right)$ and hence $a_{v}^{(1)}$ lies in the image of $E\left(F_{v}\right)$ under $\partial_{m}$. Then we can lift, by the diagram where id is the identity map,

$$
\begin{array}{ccc}
E\left(F_{v}\right) & \stackrel{\partial_{m}}{\rightarrow} & H^{1}\left(F_{v}, E_{m}\right) \\
\operatorname{id} \uparrow & & \uparrow n \\
E\left(F_{v}\right) & \stackrel{\partial_{m n}}{\rightarrow} & H^{1}\left(F_{v}, E_{m n}\right)
\end{array}
$$

$a_{v}^{(1)} \in H^{1}\left(F_{v}, E_{m}\right)$ to an element $a_{v, 1}^{(1)} \in H^{1}\left(F_{v}, E_{m n}\right)$ so that $n a_{v, 1}^{(1)}=a_{v}^{(1)}$ and $a_{v, 1}^{(1)}$ is in the image of $E\left(F_{v}\right)$ under $\partial_{m n}$.

Let $\beta$ be a cocycle in $\operatorname{Cocy}^{1}\left(F, E_{m}\right)$ representing $a^{(1)} \in H^{1}\left(F, E_{m}\right)$, and lift it to a cochain $\beta_{1} \in \operatorname{Coch}^{1}\left(F, E_{m n}\right)$. Select a cocycle $\beta_{v, 1} \in \operatorname{Cocy}^{1}\left(F_{v}, E_{m n}\right)$ representing the element $a_{v, 1}^{(1)} \in H^{1}\left(F_{v}, E_{m n}\right)$, and select a cocycle $\beta^{\prime} \in \operatorname{Cocy}^{1}\left(F, E_{n}\right)$ representing $b^{(1)} \in H^{1}\left(F, E_{n}\right)$.

The coboundary $d \beta_{1}$ of $\beta_{1}$ takes values in $E_{n}$, as $\beta_{1}$ is a cochain lifting the cocycle $\beta$ with values in $E_{m}$. The cup product $d \beta_{1} \cup \beta^{\prime}$ represents an element of $H^{3}\left(\mathrm{Gal}\left(F^{\mathrm{sep}} / F\right), \mathbb{G}_{m}\right)$ where $\mathbb{G}_{m}$ is the multiplicative group scheme over $F$ and where this cup product is induced by the Weil pairing $E_{n} \times E_{n} \rightarrow \mathbb{G}_{m}$ (see Section 2.1.2). But this last cohomology group $H^{3}\left(\mathrm{Gal}\left(F^{\mathrm{sep}} / F\right), \mathbb{G}_{m}\right)$ is zero (see [10, Chapter I, Corollary 4.18 or 4.21]). Hence we have that 


$$
d \beta_{1} \cup \beta^{\prime}=d \epsilon
$$

for some 2-cochain $\epsilon \in \operatorname{Coch}^{2}\left(F, \mathbb{G}_{m}\right)$.

The cochain localized at $v$

$$
\left(\left(\beta_{v, 1}-\beta_{1, v}\right) \cup \beta_{v}^{\prime}\right)+\epsilon_{v}
$$

is a 2-cocycle in $\operatorname{Cocy}^{2}\left(F_{v}, \mathbb{G}_{m}\right)$. Denote by

$$
\operatorname{inv}_{v}: \operatorname{Br}\left(F_{v}\right)=H^{2}\left(F_{v}, \mathbb{G}_{m}\right) \rightarrow \mathbb{Q} / \mathbb{Z}
$$

the canonical isomorphism (the invariant map) given by local class field theory. Define

$$
\langle a, b\rangle_{\text {Cassels }}=\sum_{v \in \Sigma_{F}} \operatorname{inv}_{v}\left(\left(\left(\beta_{v, 1}-\beta_{1, v}\right) \cup \beta_{v}^{\prime}\right)+\epsilon_{v}\right) \in \mathbb{Q} / \mathbb{Z} .
$$

This can be checked to be independent of the selections made and defines the pairing on $\amalg(E / F)_{(\text {non- } p)}$.

REMARKS 2.3.1

(a) For this paper, only that part of the construction of the Cassels pairing in Sections 2.3.2-2.3.5 is required. This is because, under the hypotheses of the main theorems of this paper stated in Section 4.1, the subgroup $\amalg(E / K)$ of $H^{1}(K, E)$ has the following divisibility property.

Under the hypotheses and notations of the main theorems of this paper stated in Section 4.1, for all prime numbers $l \in \mathcal{P}$ where $l$ is coprime to $\operatorname{Pic}(A)$ and for any integer $n \geq 0$ there is a subgroup $H$ of $H^{1}(K, E)$ such that $l^{n} H=$ $\amalg(E / K)_{l^{\infty}}$.

This divisibility holds because for any sufficiently large positive integer $a$ the group $\amalg(E / K)_{l^{\infty}}$ is generated by the cohomology classes $\delta_{M_{0}}(c)$ where $c$ ranges over the elements of $\Lambda(a)$ (Theorem 5.6.2) and $l^{n} \delta_{M_{0}+n}(c)=\delta_{M_{0}}(c)$ if $c \in \Lambda\left(M_{0}+n\right)$ (Lemma 4.2.1(c)).

It would be interesting to have examples of elliptic curves $E / K$ and prime numbers $l$ for which $\amalg(E / K)_{l \infty}$ is nonzero but does not have this divisibility property as a subgroup of $H^{1}(K, E)$.

(b) The Cassels pairing for abelian varieties over global fields can be defined in several ways. For a more geometric construction of the pairing than that given above, see [10, Chapter I, Remark 6.11, p. 98]. For the special case of Jacobians of curves, see [10, Chapter I, Remark 6.12, p. 100]. For the construction of the pairing including the $p$-torsion part of the Tate-Shafarevich group, where $p$ is the characteristic of the base field, see [10, Chapter II, Theorem 5.6, pp. 247-248].

Part 3. The cohomology classes $\gamma_{n}(c), \delta_{n}(c)$

\subsection{The set $\mathcal{P}$ of prime numbers}

We define a set $\mathcal{P}$ of prime numbers by arithmetic conditions. For prime numbers $l$ of $\mathcal{P}$ we shall consider in Parts 4 and 5 the structure of the $l$-primary component of the Tate-Shafarevich group $\amalg(E / F)$ of elliptic curves $E / F$. 


\subsection{1.}

For a place $v \in \Sigma_{F}$ of the global field $F$, let

$F_{v}$ denote the completion of $F$ at $v$;

$F_{v}^{\text {nr }}$ denote the maximal unramified extension of the local field $F_{v}$ (that is, $F_{v}^{\mathrm{nr}}$ is the field of fractions of the strict Henselization of the valuation ring of $\left.F_{v}\right)$;

$O_{v}$ denote the discrete valuation ring of the local field $F_{v}$;

$\infty \in \Sigma_{F}$ be a place of $F$;

$K$ be a separable imaginary quadratic extension field of $F$ with respect to the place $\infty$ as in Section 1.2;

$E / F$ be an elliptic curve (as in Section 1.5);

$\mathcal{E}$ denote the Néron model over $O_{v}$ of the elliptic curve $E \times{ }_{F} F_{v} / F_{v}$;

$\mathcal{E}_{0}$ denote the closed fiber of $\mathcal{E} / O_{v}$;

$\pi_{0}\left(\mathcal{E}_{0}\right)$ be the group of connected components of $\mathcal{E}_{0}$ as a $\operatorname{Gal}\left(F_{v}^{\mathrm{nr}} / F_{v}\right)$ module.

Define similarly $K_{w}, K_{w}^{\mathrm{nr}}$ for a place $w \in \Sigma_{K}$ of the imaginary quadratic extension field $K$ of $F$.

THEOREM 3.1.1 ([10, CHAPTER I, PROPOSITION 3.8, P. 57])

Write $G=\operatorname{Gal}\left(F_{v}^{\mathrm{nr}} / F_{v}\right)$. There is an isomorphism

$$
H^{1}\left(G, E\left(F_{v}^{\mathrm{nr}}\right)\right) \cong H^{1}\left(G, \pi_{0}\left(\mathcal{E}_{0}\right)\right) .
$$

In particular, $H^{1}\left(G, E\left(F_{v}^{\mathrm{nr}}\right)\right)$ is a finite group for all $v$ and if the elliptic curve $E$ has good reduction at $v$, then $H^{1}\left(G, E\left(F_{v}^{\mathrm{nr}}\right)\right)=0$.

\section{DEFINITION 3.1.2}

Let $\mathcal{P}$ be the set of all prime numbers such that for all $l \in \mathcal{P}$ we have that

(a) $p, 2$, and the prime factors of $\left|B^{*}\right| /\left|A^{*}\right|$ are not in $\mathcal{P}$;

(b) $H^{i}\left(K\left(E_{l^{n}}\right) / K, E_{l^{n}}\right)=0$ for all integers $n \geq 1$ and for all $i \geq 0$ (see Proposition 1.12.1);

(c) the natural injection $\operatorname{Gal}\left(F\left(E_{l \infty}\right) / F\right) \rightarrow \widehat{\Gamma}_{l}$ is an isomorphism (see Section 1.11 and Igusa's Theorem 1.11.2);

(d) $H^{1}\left(K_{z}^{\mathrm{nr}} / K_{z}, E\right)_{l \infty}=0$ for all places $z$ of $K$ (see Theorem 3.1.1 above);

(e) $K$ and $F\left(E_{l \infty}\right)$ are linearly disjoint over $F$ (see Proposition 1.12.2 or [1, Proposition 7.3.10]);

(f) $\mathcal{P}$ excludes the prime numbers of the finite set $\mathcal{E}$ of Proposition 1.10.1, that is to say, we have $E(K[A])_{l^{m}}=0$ for all $l \in \mathcal{P}$, for all $m \geq 1$, where $K[A]$ is defined in Section 1.10;

(g) $\left(\begin{array}{cc}1 & 0 \\ 0 & -1\end{array}\right) \in \operatorname{Gal}\left(F\left(E_{l \infty}\right) / F\right)$ (see Proposition 1.12.2, which is a consequence of Igusa's Theorem 1.11.1).

REMARKS 3.1.3

(a) The first six conditions of Definition 3.1.2 hold for all except finitely many 
prime numbers $l$. Only condition (g) of Definition 3.1.2 fails to hold in general for all but finitely many prime numbers. The set $\mathcal{P}$ is infinite and has positive Dirichlet density.

The set $\mathcal{P}$ can therefore be obtained from the set $S$ of prime numbers provided by Proposition 1.12.2 by deleting a finite number of elements. More precisely, the set $\mathcal{P}$ consists of all but finitely many prime numbers $l$ of the form $2^{s} n+1$ where $s \geq 1$ and $n$ is odd such that $q=|k|$ is a $2^{s}$ th-power nonresidue modulo $l$ (see [1, Remarks 7.3.14(1) and Remarks 7.7.6(1)]).

(b) The set $\mathcal{P}$ of prime numbers of Definition 3.1.2 above coincides with the set of prime numbers written $\mathcal{P} \backslash \mathcal{F}$ of [1, Lemma 7.14.11]. The only difference between Definition 3.1.2 of the set of prime numbers $\mathcal{P}$ and the similar definition [1, Definition 7.10.3] is the extra hypothesis of Definition 3.1.2(f) above, which excludes from $\mathcal{P}$ the finitely many prime numbers of the exceptional set $\mathcal{E}$ of Proposition 1.10.1.

LEMMA 3.1.4

For any integers $0 \leq m \leq n$ and for all prime numbers $l \in \mathcal{P}$ the inclusion of group schemes $E_{l^{m}} \rightarrow E_{l^{n}}$ induces an injection of cohomology groups

$$
H^{1}\left(K, E_{l^{m}}\right) \longrightarrow H^{1}\left(K, E_{l^{n}}\right) .
$$

Proof

This follows from the long exact sequence induced by the isogeny on the finite group scheme $E_{n}$ of multiplication by $l^{m}$

$$
\begin{gathered}
0 \rightarrow E_{l^{m}}(K) \rightarrow E_{l^{n}}(K) \rightarrow E_{l^{n-m}}(K) \\
\rightarrow H^{1}\left(K, E_{l^{m}}\right) \rightarrow H^{1}\left(K, E_{l^{n}}\right) \rightarrow H^{1}\left(K, E_{l^{n-m}}\right) \rightarrow \cdots
\end{gathered}
$$

together with the nonexistence of $K$-rational $l$-torsion on $E$ (by Proposition 1.10.1 and the definition of $\mathcal{P}$, Definition 3.1.2(f)).

\subsection{2.}

We write, where the limits are set-theoretic unions by Lemma 3.1.4,

$$
H^{1}\left(K, E_{l \infty}\right)=\underset{n}{\lim } H^{1}\left(K, E_{l^{n}}\right)
$$

and

$$
\operatorname{Sel}_{l^{\infty}}(E / K)=\underset{n}{\lim _{n}} \operatorname{Sel}_{l^{n}}(E / K)
$$

\subsection{3.}

Let $\mathbb{Q}(l)$, for any prime number $l$, be the additive group of rational numbers with denominators a power of $l$; the quotient group $\mathbb{Q}(l) / \mathbb{Z}$ is a divisible abelian group where every element is annihilated by a power of $l$.

We have the exact sequence of abelian groups for all prime numbers $l \in \mathcal{P}$ where $E(K)_{\text {tors }}$ denotes the torsion subgroup of $E(K)$ 


$$
0 \longrightarrow \frac{E(K)}{E(K)_{\text {tors }}} \otimes_{\mathbb{Z}} \frac{\mathbb{Q}(l)}{\mathbb{Z}} \longrightarrow \operatorname{Sel}_{l^{\infty}}(E / K) \longrightarrow \amalg(E / K)_{l^{\infty}} \longrightarrow 0 .
$$

This exact sequence (3.1.1) is obtained from Lemma 3.1.4, the exact sequence of Section 2.2.3, and because $E(K)$ has no $l$-torsion (see Definition 3.1.2(f)).

The exact sequence (3.1.1) splits and gives the isomorphism

$$
\operatorname{Sel}_{l \infty}(E / K) \cong \frac{E(K)}{E(K)_{\text {tors }}} \otimes_{\mathbb{Z}} \frac{\mathbb{Q}(l)}{\mathbb{Z}} \oplus \amalg(E / K)_{l^{\infty}} .
$$

This holds because the abelian group $E(K) / E(K)_{\text {tors }} \otimes_{\mathbb{Z}} \mathbb{Q}(l) / \mathbb{Z}$, where $E(K)$ is a finitely generated group, is injective in the category of abelian groups.

It follows from the isomorphism (3.1.2) that $\operatorname{Sel}_{l^{m}}(E / K)$ is precisely the subgroup of $\operatorname{Sel}_{l \infty}(E / K)$ annihilated by $l^{m}$; that is to say, we have for all $m \geq 0$ and all $l \in \mathcal{P}$ that

$$
\operatorname{Sel}_{l^{m}}(E / K) \cong\left(\operatorname{Sel}_{l^{\infty}}(E / K)\right)_{l^{m}}
$$

\subsection{Frobenius elements and the set $\Lambda(n)$ of divisors}

\subsection{1.}

Let

$F$ be the function field of the curve $C / k$ as in Section 1.2;

$\infty \in \Sigma_{F}$ be a closed point of $C / k$;

$K$ be a separable imaginary quadratic extension field of $F$ with respect to $\infty$ as in Section 1.2;

$\tau \in \operatorname{Gal}(K / F)$ be the nontrivial element of the Galois group of $K / F$;

$E / F$ be an elliptic curve with conductor $I$;

$\mathcal{P}$ be the set of prime numbers associated to $E, F, K$ as in Section 3.1;

$l \in \mathcal{P}$ be a prime number in $\mathcal{P}$.

As in Section 1.12.1, for every prime number $l \neq p$, an isomorphism is fixed between $\operatorname{Gal}\left(F\left(E_{l \infty}\right) / F\right)$ and a subgroup of $\mathrm{GL}_{2}\left(\mathbb{Z}_{l}\right)$ by fixing a basis of the corresponding Tate module.

\subsection{2.}

For each integer $n \geq 1$ and the chosen $l \in \mathcal{P}$, let $\tau_{\infty} \in \operatorname{Gal}\left(K\left(E_{l^{n}}\right) / F\right)$ be the unique element of this Galois group satisfying the two conditions:

(a) $\left.\tau_{\infty}\right|_{F\left(E_{l^{n}}\right)}=\left(\begin{array}{cc}1 & 0 \\ 0 & -1\end{array}\right)$; that is to say, the restriction of $\tau_{\infty}$ to the field extension $F\left(E_{l^{n}}\right) / F$ is $\left(\begin{array}{cc}1 & 0 \\ 0 & -1\end{array}\right)$;

(b) $\left.\tau_{\infty}\right|_{K}=\tau$ is the nontrivial element of $\operatorname{Gal}(K / F)$.

The elements $\tau$ and $\tau_{\infty}$ have exact order 2 .

\subsection{3.}

For any $\mathbb{Z}[\mathrm{Gal}(K / F)]$-module $M$ on which multiplication by 2 is an isomorphism, we have a decomposition of $M$ as a sum of eigenspaces under the action of $\tau$, the 
nontrivial element of $\operatorname{Gal}(K / F)$,

$$
M \cong M^{+} \oplus M^{-}
$$

where $M^{+}$is the submodule of $M$ on which $\tau$ acts as 1 and where $M^{-}$is similarly the submodule of $M$ on which $\tau$ acts as -1 .

\section{DEFINITION 3.2.1}

(a) For a prime divisor $z \in \Sigma_{F}$, unramified in the field extension $K\left(E_{l^{n}}\right) / F$, let

$$
\operatorname{Frob}(z)
$$

denote the conjugacy class of $\operatorname{Gal}\left(K\left(E_{l^{n}}\right) / F\right)$ containing the Frobenius substitutions of the prime divisors above $z$.

(b) For $l \in \mathcal{P}$, let $\Lambda^{1}(n)$ be the set of prime divisors $z$ in $\Sigma_{F}$, of support coprime to $\infty$ and $\operatorname{Supp}(I)$ and the discriminant of $K / F$, which satisfy

$$
\operatorname{Frob}(z)=\left[\tau_{\infty}\right]
$$

where $\left[\tau_{\infty}\right]$ denotes the conjugacy class in $\operatorname{Gal}\left(K\left(E_{l^{n}}\right) / F\right)$ of $\tau_{\infty}$.

(c) For $r \geq 0$, let $\Lambda^{r}(n)$ be the set of effective divisors $z_{1}+\cdots+z_{r}$ on the affine curve $\operatorname{Spec} A$ which have $r$ prime components $z_{i}$, all of which have multiplicity 1 and belong to $\Lambda^{1}(n)$.

We conventionally put for all $n \geq 1$

$$
\Lambda^{0}(n)=\{0\},
$$

which is the set consisting of the zero divisor on $C / k$.

(d) Put

$$
\begin{aligned}
\Lambda^{r} & =\Lambda^{r}(1), \\
\Lambda(n) & =\bigcup_{r \geq 0} \Lambda^{r}(n), \\
\Lambda & =\bigcup_{n \geq 1} \Lambda(n) .
\end{aligned}
$$

We have that

$$
\Lambda^{r}(n)=\left\{\begin{array}{l|c}
z_{1}+\cdots+z_{r} \in \operatorname{Div}_{+}(A) & \begin{array}{c}
z_{1}, \ldots, z_{r} \text { are distinct prime divisors such } \\
\text { that, for all } i, z_{i} \text { is prime to } \infty, \operatorname{Supp}(I), \\
\text { and the discriminant of } K / F \text { and } \\
\operatorname{Frob}\left(z_{i}\right)=\left[\tau_{\infty}\right] \text { on } K\left(E_{l^{n}}\right)
\end{array}
\end{array}\right\} .
$$

The set $\Lambda^{r}$ has a decreasing filtration

$$
\Lambda^{r}=\Lambda^{r}(1) \supseteq \Lambda^{r}(2) \supseteq \Lambda^{r}(3) \supseteq \cdots .
$$

\section{REMARKS 3.2.2}

(a) The prime divisors in $\Lambda(n)$ are infinite in number, by the Chebotarev density theorem, and remain prime in the field extension $K / F$, and their liftings to $K$ split completely in $K\left(E_{l^{n}}\right) / K$. Furthermore, $E$ has good reduction at all prime divisors of $\Lambda(n)$. 
Note that the prime number $l \in \mathcal{P}$ is considered to be fixed and the sets $\Lambda^{r}(n)$ depend on $l$.

(b) The set $\Lambda(n)$ is defined for any prime number $l$ in $\mathcal{P}$ and contains only effective divisors on $\operatorname{Spec} A$ consisting of sums of distinct prime divisors whose corresponding Frobenius conjugacy classes in $\operatorname{Gal}\left(K\left(E_{l^{n}}\right) / F\right)$ are all the same and equal to $\left[\tau_{\infty}\right]$. This unique Frobenius conjugacy class is of a special kind; in particular, its elements have order 2 .

\subsection{4.}

For any prime number $\lambda$ distinct from the characteristic of $F$, let

$$
\rho: \operatorname{Gal}\left(F^{\mathrm{sep}} / F\right) \rightarrow \operatorname{Aut}_{\mathbb{Q}_{\lambda}}\left(T_{\lambda}(E) \otimes_{\mathbb{Z}_{\lambda}} \mathbb{Q}_{\lambda}\right)
$$

denote the Galois representation on the $\lambda$-adic Tate module $T_{\lambda}(E)$ of the elliptic curve $E$; that is to say,

$$
T_{\lambda}(E) \otimes_{\mathbb{Z}_{\lambda}} \mathbb{Q}_{\lambda}=H_{\text {ét }}^{1}\left(E \otimes_{F} F^{\text {sep }}, \mathbb{Q}_{\lambda}\right)^{*},
$$

where $*$ denotes the dual $\mathbb{Q}_{\lambda}$-vector space.

For $z$ a prime of $F$, let $I_{z}$ be an inertia subgroup of $\operatorname{Gal}\left(F^{\mathrm{sep}} / F\right)$ at $z$, and let

$$
a_{z}=\operatorname{Tr}\left(\rho(\operatorname{Frob}(z)) \mid\left(T_{\lambda}(E) \otimes_{\mathbb{Z}_{\lambda}} \mathbb{Q}_{\lambda}\right)^{I_{z}}\right) .
$$

That is to say, $a_{z}$ is the trace of the Frobenius at $z$ on the part of the Tate module invariant under $I_{z}$. Then we have $a_{z} \in \mathbb{Z}$ (see [1, Examples 5.3.18(1)]).

\section{LEMMA 3.2.3}

Suppose that $z \in \Sigma_{F}$ is a prime divisor of $F$, and suppose that $l \in \mathcal{P}$. Write $\kappa(z)$ for the residue field at $z$.

(a) If $z \in \Lambda^{1}(n)$, then we have $a_{z} \equiv|\kappa(z)|+1 \equiv 0\left(\bmod l^{n}\right)$.

(b) If $z \in \Lambda^{1}(n), \mathcal{E}_{0, z}$ denotes the closed fiber over $z$ of the Néron model of $E / F$, and $y$ is the prime divisor of $K$ lying over $z$, then we have group isomorphisms, for $\delta=+1$ or -1 ,

$$
\mathcal{E}_{0, z}(\kappa(y))_{l^{n}}^{\delta} \cong \mathcal{E}_{0, z}(\kappa(z))_{l^{n}} \cong \mathbb{Z} / l^{n} \mathbb{Z}
$$

Proof

(a) As $a_{z}$ is the trace of a Frobenius above $z$ on the Tate module of $E / F$, we have by the Grothendieck-Lefschetz trace formula, where $\kappa(z)$ is the residue field of $F$ at $z$ and $\mathcal{E}_{0, z}$ is the closed fiber over $z$ of the Néron model of $E / F$,

$$
a_{z}=|\kappa(z)|+1-\left|\mathcal{E}_{0, z}(\kappa(z))\right| .
$$

On the one hand, let $K^{\prime}$ be the field $K\left(E_{l^{n}}\right)$. The characteristic polynomial of the Frobenius Frob $_{K^{\prime} / F}(z)$ above $z$ acting on the $l$-adic Tate module of $E / F$ is equal to

$$
X^{2}-a_{z} X+|\kappa(z)|
$$


On the other hand as $z \in \Lambda^{1}(n)$, the prime $z$ is a place of good reduction of $E / F$ (see Remark 3.2.2(a)). Therefore, the characteristic polynomial of the Frobenius $\operatorname{Frob}_{K^{\prime} / F}(z)=\left[\tau_{\infty}\right]$ above $z$ acting on $E_{l^{n}}$, the group scheme of $l^{n}$ torsion points of $E$, is equal to

$$
X^{2}-1\left(\bmod l^{n}\right)
$$

by condition (a) of Section 3.2.2.

Comparing these two quadratic polynomials modulo $l^{n}$ proves the congruences in part (a) of the lemma.

(b) Let $y$ be the unique place of $K$ lying over the place $z$ of $F$ where $z \in \Lambda^{1}(n)$. Then $\kappa(y)$ is a quadratic extension of $\kappa(z)$. Furthermore, as $\operatorname{Frob}_{K^{\prime} / F}(z)=\left[\tau_{\infty}\right]$ where $\tau_{\infty}$ has order 2 (see Section 3.2.2, Remark 3.2.2(b)), the prime $y$ splits completely in the extension $K^{\prime} / K$. The map of reduction modulo a prime of $K^{\prime}$ over $z$

$$
E\left(K^{\prime}\right)_{l^{n}} \rightarrow \mathcal{E}_{0, z}(\kappa(y))_{l^{n}}
$$

is an isomorphism. Hence, we have that

$$
\mathcal{E}_{0, z}(\kappa(y))_{l^{n}} \cong\left(\frac{\mathbb{Z}}{l^{n} \mathbb{Z}}\right)^{2} .
$$

As $l$ is an odd prime number and the roots \pm 1 of the characteristic polynomial $X^{2}-1$ of $\tau_{\infty}$ on $E_{l^{n}}$ are rational over the prime field $\mathbb{Z} / l \mathbb{Z}$, the action of $\tau_{\infty}$ on $\mathcal{E}_{0, z}(\kappa(y))_{l^{n}}$ decomposes into a sum over the eigenspaces of $\tau_{\infty}$. Hence, we have that for $\delta= \pm 1$

$$
\mathcal{E}_{0, z}(\kappa(y))_{l^{n}}^{\delta} \cong \mathbb{Z} / l^{n} \mathbb{Z}
$$

Furthermore, we have that

$$
\mathcal{E}_{0, z}(\kappa(y))^{+}=\mathcal{E}_{0, z}(\kappa(z)) .
$$

The result follows from this.

\section{REMARKS 3.2.4}

(a) Let $z \in \Sigma_{F}$ be a prime divisor which is coprime to $\infty, \operatorname{Supp}(I)$, and the discriminant of $K / F$ and which is inert in the field extension $K / F$. Then it can be shown, in a similar way to the proof of Lemma 3.2.3, that $z \in \Lambda^{1}(n)$ if and only if

$$
a_{z} \equiv|\kappa(z)|+1 \equiv 0\left(\bmod l^{n}\right)
$$

and the Frobenius $\operatorname{Frob}(z)$ does not act as a homothety on $T_{l}(E) \otimes_{\mathbb{Z}_{l}} \mathbb{Z} / l^{n} \mathbb{Z}$ where $a_{z}$ is the trace of the Frobenius $\operatorname{Frob}(z)$ on the Tate module as in Section 3.2.4.

(b) Fix a prime $l \in \mathcal{P}$. For two integers $a, b \in \mathbb{Z}$ denote by $v_{l}(a, b)$ the valuation at $l$ of the greatest common divisor of $a$ and $b$. For a prime divisor $z$ of $\operatorname{Spec} A$ define $\alpha(z)$ by the equation

$$
\alpha(z)=v_{l}\left(|\kappa(z)|+1, a_{z}\right)
$$


where as in Section 3.2.4

$$
a_{z}=|\kappa(z)|+1-\left|\mathcal{E}_{0}(\kappa(z))\right|
$$

and where $\mathcal{E}_{0}$ is the closed fiber of the Néron model of $E$ at $z$.

The prime divisors $z$ of $\operatorname{Spec} A$ in the set $\Lambda^{1}$ have the property that they are coprime to $I$, remain prime in $K / F$, and satisfy

$$
\alpha(z)=v_{l}\left(|\kappa(z)|+1, a_{z}\right) \geq 1 .
$$

(c) If $r>0$ and $c \in \Lambda^{r}$, then we write

$$
\alpha(c)=\min _{z \in \operatorname{Supp}(c)} \alpha(z), \quad \alpha(0)=+\infty .
$$

We have the equation

$$
\Lambda^{r}(n)=\left\{c \in \Lambda^{r} \mid \alpha(c) \geq n\right\},
$$

where $n$ is any integer $\geq 1 ; \alpha(c)$ is the greatest integer $n$ such that $c \in \Lambda^{r}(n)$. The set $\Lambda^{r}$ is then equipped with a decreasing filtration

$$
\Lambda^{r}=\Lambda^{r}(1) \supseteq \Lambda^{r}(2) \supseteq \Lambda^{r}(3) \supseteq \cdots .
$$

(d) In the notation of the monograph [1, Section 7.11], we have $\Lambda(n)=\mathcal{D}_{l^{n}}$.

\subsection{A refined Hasse principle for finite group schemes}

The finite group schemes in question are $E_{l^{n}} / F$, and the Hasse principle concerns the localization at places of $F$ of finite groups of their principal homogenous spaces.

\subsection{1.}

Suppose that

$E$ is an elliptic curve defined over $F$;

$K$ is an imaginary quadratic field over $F$ with respect to $\infty$;

$l \in \mathcal{P}$ is a necessarily odd prime number in the set $\mathcal{P}$ (see Section 3.1);

$n \geq 1$ is an integer;

$L_{n}$ is the field $K\left(E_{l^{n}}\right)$ which is Galois over $F$.

\section{LEMMA 3.3.1}

The restriction map from $K$ to $L_{n}$ induces an isomorphism

$$
H^{1}\left(K, E_{l^{n}}\left(L_{n}\right)\right) \stackrel{\cong}{\longrightarrow} \operatorname{Hom}_{\operatorname{Gal}\left(L_{n} / K\right)}\left(\operatorname{Gal}\left(L_{n}^{\mathrm{ab}} / L_{n}\right), E_{l^{n}}\left(L_{n}\right)\right),
$$

where $L_{n}^{\mathrm{ab}}$ is the maximal separable abelian extension of $L_{n}$.

Proof

The Hochschild-Serre spectral sequence, where as in Section 3.3.1 $L_{n}=K\left(E_{l^{n}}\right)$,

$$
H^{p}\left(L_{n} / K, H^{q}\left(L_{n}, E_{l^{n}}\left(L_{n}\right)\right)\right) \Rightarrow H^{p+q}\left(K, E_{l^{n}}\left(L_{n}\right)\right)
$$


gives rise to a short exact sequence of low-degree terms

$$
\begin{aligned}
& 0 \rightarrow H^{1}\left(L_{n} / K,\right.\left.E_{l^{n}}\left(L_{n}\right)\right) \rightarrow H^{1}\left(K, E_{l^{n}}\left(L_{n}\right)\right) \rightarrow H^{1}\left(L_{n}, E_{l^{n}}\left(L_{n}\right)\right)^{\operatorname{Gal}\left(L_{n} / K\right)} \\
& \rightarrow H^{2}\left(L_{n} / K, E_{l^{n}}\left(L_{n}\right)\right) \rightarrow H^{2}\left(K, E_{l^{n}}\left(L_{n}\right)\right) .
\end{aligned}
$$

The two cohomology groups $H^{1}\left(L_{n} / K, E_{l^{n}}\left(L_{n}\right)\right)$ and $H^{2}\left(L_{n} / K, E_{l^{n}}\left(L_{n}\right)\right)$ are zero by the definition of $\mathcal{P}$ (see Definition 3.1.2(b)); hence, this exact sequence shows that the restriction map of the lemma is an isomorphism. We have that

$$
H^{1}\left(K, E_{l^{n}}\left(L_{n}\right)\right) \rightarrow H^{1}\left(L_{n}, E_{l^{n}}\left(L_{n}\right)\right)^{\operatorname{Gal}\left(L_{n} / K\right)}
$$

is an isomorphism of $\operatorname{Gal}\left(L_{n} / F\right)$-modules. The isomorphism of the lemma follows immediately.

PROPOSITION 3.3.2

If $S$ is a finite subgroup of $H^{1}\left(K, E_{l^{n}}\right)$, then there is a finite abelian extension $L_{S, n}$ of $L_{n}$, an isomorphism of $\operatorname{Gal}\left(L_{n} / K\right)$-modules

$$
\begin{aligned}
\operatorname{Gal}\left(L_{S, n} / L_{n}\right) & \cong \operatorname{Hom}\left(S, E_{l^{n}}\left(L_{n}\right)\right), \\
\sigma & \mapsto \phi_{\sigma},
\end{aligned}
$$

and an isomorphism of abelian groups (if $S$ is a $\mathbb{Z}[\mathrm{Gal}(K / F)]$-module, then an isomorphism of $\mathbb{Z}[\mathrm{Gal}(K / F)]$-modules)

$$
S \cong \operatorname{Hom}_{\operatorname{Gal}\left(L_{n} / K\right)}\left(\operatorname{Gal}\left(L_{S, n} / L_{n}\right), E_{l^{n}}\left(L_{n}\right)\right) .
$$

For the proof of Proposition 3.3.2, see [1, Corollary 7.18.10]. Note that the set of prime numbers $\mathcal{P} \backslash F$ of $[1,(7.18 .1)$ and Corollary 7.18.10] coincides with the set $\mathcal{P}$ of prime numbers defined in Section 3.1 above.

\subsection{2.}

For a prime divisor $v$ of $K$, denote by

$$
\operatorname{res}_{v}: H^{1}\left(K, E_{l^{n}}\right) \longrightarrow H^{1}\left(K_{v}, E_{l^{n}}\right)
$$

the restriction homomorphism at $v$ where $K_{v}$ is the completion of $K$ at $v$.

Let $S$ be a finite subgroup of $H^{1}\left(K, E_{l^{n}}\right)$. For $s \in S$ and $v$ a place of $K$, we have in the notation of Proposition 3.3.2 applied to the subgroup $S$

$$
\operatorname{res}_{v}(s)=0 \Leftrightarrow \phi_{\sigma}(s)=0 \text { for all } \sigma \in D_{v^{\prime}},
$$

where $v^{\prime}$ is a prime divisor of $L_{n}$ above $v, D_{v^{\prime}}$ is the decomposition group of a prime divisor of $L_{S, n}$ above $v^{\prime}$, and $\phi_{\sigma} \in \operatorname{Hom}\left(S, E_{l^{n}}\left(L_{n}\right)\right)$ as in (3.3.1).

\subsection{3.}

Let $\tau_{\infty} \in \operatorname{Gal}\left(L_{n} / F\right)$ be the element of order 2 of Section 3.2.2. The element $\tau_{\infty}$ acts as -1 on the $l^{n}$ th roots of unity and preserves the Weil pairing on $E_{l^{n}}$. (With reference to the exact sequence (1.11.1), $\operatorname{det}\left(\tau_{\infty}\right)=-1$ acts on the $l^{n}$ th roots of unity.) 
We obtain (see Section 3.2.3), because $l$ is odd, the decompositions into eigenspaces under the action of $\tau_{\infty}$

$$
\begin{aligned}
H^{1}\left(K, E_{l^{n}}\right) & \cong H^{1}\left(K, E_{l^{n}}\right)^{+} \oplus H^{1}\left(K, E_{l^{n}}\right)^{-}, \\
E_{l^{n}}\left(L_{n}\right) & \cong E_{l^{n}}\left(L_{n}\right)^{+} \oplus E_{l^{n}}\left(L_{n}\right)^{-} .
\end{aligned}
$$

We obtain that the +1 eigenspace

$$
\operatorname{Hom}\left(H^{1}\left(K, E_{l^{n}}\right), E_{l^{n}}\left(L_{n}\right)\right)^{+}
$$

is isomorphic to the profinite group of $\mathbb{Z}\left[\tau_{\infty}\right]$-homomorphisms from $H^{1}\left(K, E_{l^{n}}\right)$ to $E_{l^{n}}\left(L_{n}\right)$ and hence we obtain an isomorphism between this $\tau_{\infty}$-invariant subgroup and the profinite Pontryagin dual of the discrete torsion abelian group $H^{1}\left(K, E_{l^{n}}\right)$; namely, we have an isomorphism, where a basis of $T_{l}(E)$ is fixed as in Section 1.12.1,

$$
\operatorname{Hom}\left(H^{1}\left(K, E_{l^{n}}\right), E_{l^{n}}\left(L_{n}\right)\right)^{+} \cong H^{1}\left(K, E_{l^{n}}\right)^{*},
$$

where the Pontryagin dual is given by

$$
H^{1}\left(K, E_{l^{n}}\right)^{*}=\operatorname{Hom}\left(H^{1}\left(K, E_{l^{n}}\right), \mathbb{Q} / \mathbb{Z}\right) .
$$

For a finite subgroup $S$ of $H^{1}\left(K, E_{l^{n}}\right)$ there is similarly an isomorphism

$$
\widehat{S} \cong \operatorname{Hom}\left(S, E_{l^{n}}\left(L_{n}\right)\right)^{+} .
$$

\section{PROPOSITION 3.3.3}

Let $S$ be a finite subgroup of $H^{1}\left(K, E_{l^{n}}\right)$, and let $\chi \in \widehat{S}$. For any integer $t \geq$ $n$, there is a set of positive Dirichlet density of prime divisors $z \in \Lambda^{1}(t)$ of $F$ unramified in $L_{t}=K\left(E_{l^{t}}\right)$ such that

$$
\chi=\phi_{\operatorname{Frob}\left(z^{\times}\right)}
$$

for some prime divisor $z^{\times}$of $L_{t}$ lying above $z$, where $\phi_{\operatorname{Frob}\left(z^{\times}\right)} \in \operatorname{Hom}\left(S, E_{l^{t}}\left(L_{t}\right)\right)^{+}$ is as in (3.3.1).

Proof

By Lemma 3.1.4, there is an injection of cohomology groups for all $t \geq n$

$$
H^{1}\left(K, E_{l^{n}}\right) \rightarrow H^{1}\left(K, E_{l^{t}}\right)
$$

obtained from the inclusion of finite group schemes $E_{l^{n}} \subseteq E_{l^{t}}$. The finite subgroup $S$ is then a subgroup of $H^{1}\left(K, E_{l^{t}}\right)$ for all $t \geq n$. Applying Proposition 3.3.2 to $S$ as a subgroup of $H^{1}\left(K, E_{l^{t}}\right)$ we obtain the abelian extension $L_{S, t} / L_{t}$ where we write $L_{t}=K\left(E_{l^{t}}\right)$.

By Proposition 3.3.2 there is an element $\sigma \in \operatorname{Gal}\left(L_{S, t} / L_{t}\right)$ such that

$$
\chi=\phi_{\sigma} .
$$

We have that

$$
\operatorname{Gal}\left(L_{S, t} / L_{t}\right)^{+} \cong \widehat{S}
$$


from the isomorphism of (3.3.1) and the isomorphism of Section 3.3.3

$$
\widehat{S} \cong \operatorname{Hom}\left(S, E_{l^{t}}\left(L_{t}\right)\right)^{+} .
$$

In particular, we have that $\phi_{\sigma}^{\tau_{\infty}}=\phi_{\sigma}$. As the order of $\operatorname{Gal}\left(L_{S, t} / L_{t}\right)$ is odd, we then have that $\sigma=\rho^{\tau_{\infty}} . \rho$ for some $\rho \in \operatorname{Gal}\left(L_{S, t} / L_{t}\right)$. By the Chebotarev density theorem there is a set of positive Dirichlet density of prime divisors $z \in \Sigma_{F}$ of $F$ such that $\operatorname{Frob}(z)$ in $\operatorname{Gal}\left(L_{S, t} / F\right)$ contains $\tau_{\infty} \rho$ and where $z$ is unramified in $L_{S, t} / F$. Note that the finitely many prime divisors ramified in the field extension $L_{S, t} / F$ depend only on $S$ and not on $t$.

Since the restriction of $\tau_{\infty} \rho$ to $L_{t}$ is $\tau_{\infty}$, we have $z \in \Lambda^{1}(t)$. We then have that $z$ has residue class extension degree 2 in $L_{t} / F$ for any prime divisor above $z$ in $L_{t}$. Hence for any $z^{\times}$, a prime divisor of $L_{t}$ lying above $z$, we have that

$$
\operatorname{Frob}\left(z^{\times}\right)=\left(\tau_{\infty} \rho\right)^{2}=\rho^{\tau_{\infty}} \cdot \rho=\sigma .
$$

\section{PROPOSITION 3.3.4}

Let $S$ be a finite subgroup of $H^{1}\left(K, E_{l^{n}}\right)$, and let $\chi \in \widehat{S}$. Then for any integer $t \geq 1$ there is a set of positive Dirichlet density of prime divisors $z \in \Lambda^{1}(t)$ such that, for the prime divisor $y$ of $K$ lying over $z$, there is a commutative diagram of group homomorphisms

$$
\begin{gathered}
S \quad \stackrel{\text { res }_{y}}{\longrightarrow} \quad \operatorname{res}_{y}(S) \\
\chi \searrow \quad \cong \downarrow \psi
\end{gathered}
$$

where $\psi$ is an isomorphism of finite cyclic groups.

\section{Proof}

As $\Lambda^{1}(m), m \geq 1$, is a decreasing filtration of $\Lambda^{1}(1)$, we may assume that $t \geq n$. By Lemma 3.1.4, there is an injection of cohomology groups for all $t \geq n$

$$
H^{1}\left(K, E_{l^{n}}\right) \rightarrow H^{1}\left(K, E_{l^{t}}\right)
$$

obtained from the inclusion of finite group schemes $E_{l^{n}} \subseteq E_{l^{t}}$. The finite subgroup $S$ is then a subgroup of $H^{1}\left(K, E_{l^{t}}\right)$ for all $t \geq n$.

Select the prime divisor $z \in \Sigma_{F}$ as in Proposition 3.3.3 applied to $S, \chi$ and where the divisors $z$ are different from the finitely many prime divisors of $F$ where the cohomology classes of the finite group $S$ ramify, that is to say, the finitely many prime divisors where the field extension $L_{S, t} / F$ of (3.3.1) is ramified. For such a $z$, let $y$ be the prime divisor of $K$ above $z$.

The decomposition group of $y$ in $\operatorname{Gal}\left(L_{S, t} / K\right)$ is generated by the Frobenius element $\operatorname{Frob}(y)$ as the field extension $L_{S, t} / K$ is unramified at $y$. By Section 3.3.2 we then have that

$$
\operatorname{ker}\left(S \stackrel{\mathrm{res}_{y}}{\longrightarrow} H^{1}\left(K_{y}, E_{l^{t}}\right)\right)=\operatorname{ker}(\chi)
$$


That is to say, the kernel in $S$ of the restriction homomorphism at $y$ of the classes of $S$ is equal to $\operatorname{ker}(\chi)$. Hence, the two finite cyclic groups $\operatorname{res}_{y}(S), \chi(S)$ are isomorphic and we obtain the isomorphism $\psi$ of the commutative diagram of the proposition.

COROLLARY 3.3.5

We have that

$$
\begin{aligned}
& \Lambda^{r}(n) \quad \text { is infinite for all integers } r, n \geq 1 ; \\
& \Lambda^{0}(n)=\{0\} \quad \text { for all } n \geq 1 .
\end{aligned}
$$

Proof

We have from Proposition 3.3.4 that $\Lambda^{1}(n)$ has infinitely many elements for all integers $n \geq 1$. As $\Lambda^{r}(n)$ consists of all sums of $r$ distinct prime divisors of $\Lambda^{1}(n)$ (see Definition 3.2.1) and as $\Lambda^{0}(n)=\{0\}$, the corollary then follows.

\section{REMARK 3.3.6}

Proposition 3.3.4 implies that, under the hypotheses of the proposition, the kernel of the natural homomorphism

$$
\psi: H^{1}\left(K, E_{l^{n}}\right) \rightarrow \prod_{z \in \Lambda^{1}(n)} H^{1}\left(K_{y(z)}, E_{l^{n}}\right)
$$

is zero, where $y(z)$ is the place of $K$ over $z \in \Lambda^{1}(n)$. To show this, it is sufficient to apply the proposition to a faithful character $\chi$ of the finite cyclic group $S$ generated by any element of $H^{1}\left(K, E_{l^{n}}\right)$.

The Hasse principle for the group scheme $E_{l^{n}}$ and the set of prime divisors $\Lambda^{1}(n)$ is precisely that the kernel of the homomorphism $\psi$ is zero. It says that a principal homogeneous space of $E_{l^{n}}$ which is locally trivial at all places of $F$ in $\Lambda^{1}(n)$ must be globally trivial.

In this way, Proposition 3.3.4 is a refined form of the Hasse principle for the finite group scheme $E_{l^{n}}$. It would be interesting to know to what other group schemes the localization properties of finite subgroups of cohomology groups given in Proposition 3.3.4 also hold. For more details on the Hasse principle, see [10, pp. 142-150].

\subsection{Drinfeld-Heegner points and the cohomology classes $\gamma_{n}(c), \delta_{n}(c)$}

\subsection{1.}

Let

$E / F$ be an elliptic curve equipped with an origin, that is to say, $E / F$ is a 1-dimensional abelian variety;

$I$ be the ideal of $A$ which is the conductor of $E / F$ without the component at $\infty$; 
$\epsilon= \pm 1$ be the sign in the functional equation of the $L$-function of the elliptic curve $E / F$.

Assume that (see also [1, Sections 4.3, 4.7, or 7.6]):

$(\alpha) E / F$ has split Tate multiplicative reduction at $\infty$ (see Section 1.7 or $[1$, Section 4.7]);

( $\beta) K$ is an (separable) imaginary quadratic field extension of $F$, with respect to $\infty$, such that all primes dividing the conductor $I$ split completely in $K$.

The hypothesis $(\alpha)$ implies that $E / F$ is covered by the Drinfeld modular curve $X_{0}^{\operatorname{Drin}}(I)$ (see Section 3.4.4 below). The hypothesis $(\beta)$ ensures the existence of Drinfeld-Heegner points on $X_{0}^{\operatorname{Drin}}(I)$. The two hypotheses together ensure that there are Drinfeld-Heegner points on the elliptic curve $E / F$. Note that, from Section 3.4.6 to the end of this Section 3.4, it is assumed that $K \neq F \otimes_{\mathbb{F}_{q}} \mathbb{F}_{q^{2}}$ where $\mathbb{F}_{q}$ is the exact field of constants of $F$.

In this section we detail this construction of Drinfeld-Heegner points as well as define the cohomology classes $\gamma_{n}(c), \delta_{n}(c)$.

\subsection{2.}

Let $\lambda$ be any prime number of $\mathbb{Z}$ distinct from the characteristic of $F$. Let $\rho$ be the 2-dimensional $\lambda$-adic representation of $\operatorname{Gal}\left(F^{\mathrm{sep}} / F\right)$ corresponding to $E$ where $F^{\text {sep }}$ is the separable closure of $F$; that is to say, $\rho$ is the continuous homomorphism

$$
\rho: \operatorname{Gal}\left(F^{\mathrm{sep}} / F\right) \rightarrow \operatorname{End}_{\mathbb{Q}_{\lambda}}\left(H_{\text {ét }}^{1}\left(E \otimes_{F} F^{\mathrm{sep}}, \mathbb{Q}_{\lambda}\right)\right) .
$$

For each place $v$ of $F$ put

$$
a_{v}=\operatorname{Tr}\left(\rho(\operatorname{Frob}(v)) \mid H_{\text {ét }}^{1}\left(E \otimes_{F} F^{\text {sep }}, \mathbb{Q}_{\lambda}\right)^{T_{v}}\right)
$$

where $T_{v}$ is the inertia subgroup of $\operatorname{Gal}\left(F^{\mathrm{sep}} / F\right)$ over $v$. The representation $\rho$ satisfies (see [1, Example 5.3.18])

$$
a_{v} \in \mathbb{Z} \text { for all } v \in \Sigma_{F} \text {. }
$$

\subsection{3.}

Let

$B$ be the integral closure of $A$ in $K$;

$O_{c}$ be the order of $K$, with respect to $A$, and of conductor $c$ for any divisor $c \in \operatorname{Div}_{+}(A)$ (see [1, Section 2.2]);

$\tau$ be the nontrivial element of $\operatorname{Gal}(K / F)$;

$I B=I_{1} I_{2}$ be a factorization of ideals of $B$ where $I_{1}, I_{2}$ are ideals of $B$ such that $I_{2}^{\tau}=I_{1}$ where $I$ is the ideal of $A$ which is the conductor of $E / F$ without the place at $\infty$, as in Section 3.4.1; such a factorization exists because of the hypothesis that the prime ideal components of $I$ split completely in $K / F$ (by hypothesis $(\beta)$ of Section 3.4.1). 
As in Section 1.4, $K[c]$ denotes the ring class field of $K$ of conductor $c \in$ $\operatorname{Div}_{+}(A)$. In particular, $K[0]$ is the Hilbert class field of $K$; that is to say, $K[0]$ is the maximal unramified abelian extension of $K$ which is split completely at $\infty$.

\subsection{4.}

Let $\mathcal{H}(\rho)$ be the Heegner module of $\rho$ and $K / F$ with exceptional set of primes those dividing $I$ and the place $\infty$ with coefficients in $\mathbb{Z}$ (see [1, Section 5.3]), where $I$ is the conductor of $E$, without the component at $\infty$.

As in Section 1.7 (see also [1, Section 4.7 and Appendix B]), there is a finite surjective morphism of curves over $F$ under the hypothesis $(\alpha)$,

$$
\pi: X_{0}^{\text {Drin }}(I) \rightarrow E \text {. }
$$

We may translate $\pi$ in the group scheme $E$ so that $\pi^{-1}(0)$ consists of at least one cusp of $X_{0}^{\operatorname{Drin}}(I)$ (as in [1, (4.8.1)]); this rigidifies the map $\pi$. The cusps of the modular curve $X_{0}^{\text {Drin }}(I)$ generate a torsion subgroup of the Jacobian of this curve (see [1, Theorem 2.4.9]).

Let $a$ be a divisor class in the Picard group $\operatorname{Pic}\left(O_{c}\right)$ of the order $O_{c}$. Assume that $c$ and $I$ are coprime. Then there is a Drinfeld-Heegner point

$$
\left(a, I_{1}, c\right) \in X_{0}^{\text {Drin }}(I)(K[c])
$$

which is a noncuspidal point of $X_{0}^{\operatorname{Drin}}(I)$ and is rational over the ring class field $K[c]$.

This point $\left(a, I_{1}, c\right)$ is constructed as follows. Fix an embedding $K \rightarrow \widehat{\bar{F}}_{\infty}$ where $\widehat{\bar{F}}_{\infty}$ is the completion of the algebraic closure of $F_{\infty}$, which is the completion of $F$ at $\infty$. Let $L$ be a projective $O_{c}$-module of rank 1 in the class $a$ and contained as a lattice in $\widehat{\bar{F}}_{\infty}$. Then $I_{1}\left(O_{c}\right)=I_{1} \cap O_{c}$ is an invertible ideal of $O_{c}$ and $L^{\prime}=I_{1}\left(O_{c}\right)^{-1} L$ is a projective $O_{c}$-module of rank 1 contained as a lattice in $\widehat{\bar{F}}_{\infty}$. Let $D$ and $D^{\prime}$ be the rank 2 Drinfeld modules for $A$ over the field $\widehat{\bar{F}}_{\infty}$ corresponding, respectively, to the lattices $L$ and $L^{\prime}$. Then $D$ and $D^{\prime}$ have general characteristic and complex multiplication by $O_{c}$. The inclusion of $O_{c}$-modules $L \subset L^{\prime}$ corresponds to an $I$-cyclic isogeny $f: D \rightarrow D^{\prime}$, as its kernel is isomorphic as an $A$-module to $O_{c} / I_{1}\left(O_{c}\right) \cong A / I$. The pair $(D, \operatorname{ker}(f))$ defines the point $\left(a, I_{1}, c\right)$ on $X_{0}^{\operatorname{Drin}}(I)\left(\widehat{\bar{F}}_{\infty}\right)$. That this point $\left(a, I_{1}, c\right)$ is defined over $K[c]$ results from the main theorem of complex multiplication (see [1, Section 4.3] for more details).

\subsection{5.}

The image

$$
\pi\left(a, I_{1}, c\right) \in E(K[c])
$$

is a Drinfeld-Heegner point of $E$ rational over the ring class field $K[c]$ and is written in the notation of [1, Section 4.8] as

$$
\left(a, I_{1}, c, \pi\right) \in E(K[c]) .
$$


By [1, Example 5.3.18] there is a homomorphism of discrete $\operatorname{Gal}\left(K^{\mathrm{sep}} / K\right)$ modules

$$
\begin{aligned}
\mathcal{H}(\pi): \mathcal{H}(\rho)^{(0)} & \rightarrow E\left(F^{\mathrm{sep}}\right), \\
\langle a, c\rangle & \mapsto\left(a, I_{1}, c, \pi\right)
\end{aligned}
$$

for all $c \in \operatorname{Div}_{+}(A)$ coprime to $I, a \in \operatorname{Pic}\left(O_{c}\right)$. The image of this homomorphism $\mathcal{H}(\pi)$ consists of the $\mathbb{Z}$-linear combinations of Drinfeld-Heegner points of $E$ rational over all the ring class fields $K[c]$ for all $c$.

Let $\langle 0,0\rangle$ be the element of the Heegner module $\mathcal{H}(\rho)^{(0)}$ given by the principal class of $\operatorname{Pic}(B)$, where $B$ is the integral closure of $A$ in $K$. Let

$$
\left(0, I_{1}, 0, \pi\right)=\mathcal{H}(\pi)(\langle 0,0\rangle)
$$

be the corresponding Drinfeld-Heegner point of $E(K[0])$ (see [1, (4.8.2)]). Let

$$
P_{0}=\operatorname{Tr}_{K[0] / K}\left(0, I_{1}, 0, \pi\right) \in E(K) .
$$

That is to say, $P_{0}$ is the trace from $K[0]$ to $K$ of the point $\left(0, I_{1}, 0, \pi\right)$; the point $P_{0}$ belongs to $E(K)$ and the point $\left(0, I_{1}, 0, \pi\right)$ belongs to $E(K[0])$.

\subsection{6.}

We now impose for the rest of this section the hypothesis that $K \neq F \otimes_{\mathbb{F}_{q}} \mathbb{F}_{q^{2}}$ where $\mathbb{F}_{q}$ is the exact field of constants of $F$; that is to say, $K$ is not obtained from $F$ by ground field extension.

Let $c \in \Lambda(1)$; that is to say, $c$ is a sum of distinct prime divisors of $\Lambda^{1}(1)$ with multiplicity 1 . Let

$$
y_{c}=\pi\left(0, I_{1}, c\right) \in E(K[c]),
$$

so that $y_{c}=\left(0, I_{1}, c, \pi\right)$. The field $K[0]$ is the Hilbert class field of $K$. Let

$$
G_{c}=\operatorname{Gal}(K[c] / K[0]) .
$$

As $K \neq F \otimes_{\mathbb{F}_{q}} \mathbb{F}_{q^{2}}$ by hypothesis, we have that $B^{*}=A^{*}$, where $A^{*}, B^{*}$ are the unit groups of $A, B$, and hence (by [1, (2.3.8), p. 19]) there is a group isomorphism

$$
G_{c} \cong \prod_{z} G_{z}
$$

where the product runs over the prime divisors $z$ in the support of $c$ and

$$
G_{z}=\operatorname{Gal}(K[c] / K[c-z])
$$

is a cyclic group of order $|\kappa(z)|+1$ as $z$ is inert in $K / F$ (by [1, (2.3.12), p. 20]). Fix a generator $\sigma_{z}$ of the cyclic group $G_{z}$ for all prime divisors $z \in \Lambda^{1}(1)$.

\subsection{7.}

Write for any prime divisor $z \in \Lambda^{1}(1)$ of $F$, where $D_{z} \in \mathbb{Z}\left[G_{z}\right]$,

$$
D_{z}=-\sum_{i=1}^{|\kappa(z)|} i . \sigma_{z}^{i} .
$$


Here $D_{z}$ is the Kolyvagin element of the map $h_{z}: G_{z} \rightarrow \mathbb{Z}$ where $\sigma_{z}^{-i} \mapsto-i$ for all $0 \leq i \leq|\kappa(z)|$. Note the minus sign here which agrees with the Kolyvagin elements of [1, Chapter 5, pp. 175-178].

\subsection{8.}

For any divisor $c \in \Lambda(1)$, put

$$
D_{c}=\prod_{z \in \operatorname{Supp}(c)} D_{z}
$$

where $D_{c} \in \mathbb{Z}\left[G_{c}\right]$. Then $D_{c}$ is the Kolyvagin element of the map $h: G_{c} \rightarrow \mathbb{Z}$ given by $h=\prod_{z \in \operatorname{Supp}(z)} h_{z}$. Let

$$
\mathcal{G}_{c}=\operatorname{Gal}(K[c] / K),
$$

where there is an exact sequence of finite abelian groups

$$
0 \longrightarrow G_{c} \longrightarrow \mathcal{G}_{c} \longrightarrow \operatorname{Gal}(K[0] / K) \longrightarrow 0 .
$$

Let $\mathcal{S}$ be a set of coset representatives for $G_{c}$ in $\mathcal{G}_{c}$. Define the point $P_{c} \in E(K[c])$ by

$$
P_{c}=\sum_{s \in \mathcal{S}} s D_{c} y_{c}
$$

where $y_{c}=\pi\left(0, I_{1}, c\right)$ is the element of $E(K[c])$ in Section 3.4.6.

\subsection{9.}

Suppose now that $c \in \Lambda(n)$. We write $P_{c}\left(\bmod l^{n}\right)$ for the image of $P_{c}$ in the quotient group $l^{n} E(K[c])$. Then we have that $P_{c}\left(\bmod l^{n}\right)$ belongs to

$$
P_{c}\left(\bmod l^{n}\right) \in\left({ }_{l^{n}} E(K[c])\right)^{\mathcal{G}_{c}} .
$$

This inclusion follows immediately from the formula in $\mathbb{Z}\left[G_{c}\right]$

$$
\left(\sigma_{z}-1\right) D_{z}=-|G(c / c-z)|+\sum_{g \in G(c / c-z)} g
$$

for all $z \in \operatorname{Supp}(c)$ and that $|G(c / c-z)|=|\kappa(z)|+1$ is divisible by $l^{n}$ for all $z \in \operatorname{Supp}(c)$. (For a detailed proof of this inclusion $P_{c}\left(\bmod l^{n}\right) \in\left({ }_{l^{n}} E(K[c])\right)^{\mathcal{G}_{c}}$ see [1, Lemma 7.14.9 and Lemma 7.14.11].) Furthermore, we have that

$$
P_{0}=\operatorname{Tr}_{K[0] / K}\left(y_{0}\right) \in E(K),
$$

where $y_{0}$ is defined in Section 3.4.6 and where this notation $P_{0}$ agrees with that of (3.4.1). The point $P_{c}\left(\bmod l^{n}\right)$ in $E(K[c]) / l^{n} E(K[c])$ coincides with the point denoted $P_{c}$ in the book [1, Notation 7.14.10(iii) and Lemma 7.14.11].

3.4.10.

Let $S$ be the ring $\mathbb{Z} / m \mathbb{Z}$ where $m$ is any nonzero integer. The morphism of multiplication by $m$ on the elliptic curve $E$ then provides for any divisor $c$ of $\operatorname{Div}_{+}(A)$ prime to $I$ the following commutative diagram with exact rows and an 
exact right-hand column (see [1, Section 7.14.5]):

$$
\begin{aligned}
& 0 \\
& \downarrow \\
& H^{1}(K[c] / K, E(K[c]))_{m} \\
& \inf \downarrow \\
& 0 \rightarrow \quad{ }_{m} E(K) \quad \rightarrow \quad H^{1}\left(K, E_{m}\right) \quad \stackrel{j}{\rightarrow} \quad H^{1}(K, E)_{m} \quad \rightarrow \quad 0 \\
& \downarrow \quad \text { res } \downarrow \text { quasi-isom. } \quad \text { res } \downarrow \\
& 0 \rightarrow\left({ }_{m} E(K[c])\right)^{\mathcal{G}_{c}} \stackrel{\partial}{\rightarrow} H^{1}\left(K[c], E_{m}\right)^{\mathcal{G}_{c}} \quad \rightarrow \quad H^{1}(K[c], E)_{m}^{\mathcal{G}_{c}} \\
& f \uparrow \\
& \left(\mathcal{H}_{c, S}^{(0)}\right)^{\mathcal{G}_{c}}
\end{aligned}
$$

Here $\left(\mathcal{H}_{c, S}^{(0)}\right)$ is the $c$-component Heegner module with coefficients in the ring $S$ (see [1, (7.11.3) and Section 5.3]).

Let $\mathcal{E}$ be the finite exceptional set of prime numbers of Propositions 1.10 .1 and 1.10.2. The middle restriction homomorphism here in (3.4.2) is a quasiisomorphism of quasigroups in $\left[\mathbf{N}^{(p)}\right]_{\mathbb{Z}}$ where the finite exceptional set of prime numbers is $\mathcal{E}$ and is independent of $c$ (see Propositions 1.10.1 and 1.10.2, or alternatively [1, Proposition 7.14.2]). In particular, this middle restriction homomorphism is an isomorphism for all integers $m$ which are powers of prime numbers of $\mathcal{P}$ (Definition 3.1.2) as $\mathcal{P}$ excludes the finitely many prime numbers of $\mathcal{E}$.

The map $f$ is obtained by sending a generator $\langle a, c\rangle$ of $\mathcal{H}_{c, S}^{(0)}$ to its image $\left(a, I_{1}, c, \pi\right) \in E(K[c])$ as in Sections 3.4.4 and 3.4.5.

This diagram (3.4.2) then provides the Heegner homomorphism, for all integers $m \in \mathbb{N}$ prime to $\mathcal{E}$,

$$
\left(\mathcal{H}_{c, S}^{(0)}\right)^{\mathcal{G}_{c}} \rightarrow H^{1}(K, E)
$$

whose image belongs to $H^{1}(K[c] / K, E(K[c]))_{m}$.

\subsubsection{1.}

Take $m=l^{n}$ where $l \in \mathcal{P}$ so that the middle quasi-isomorphism in diagram (3.4.2) is an isomorphism. From the diagram (3.4.2), define $\gamma_{n}(c)$ and $\delta_{n}(c)$ by the formulae

$$
\begin{aligned}
& P_{c}\left(\bmod l^{n}\right) \in\left(l^{n} E(K[c])\right)^{\mathcal{G}_{c}} ; \\
& \gamma_{n}(c) \text { is the image of } P_{c}\left(\bmod l^{n}\right) \text { in } H^{1}\left(K, E_{l^{n}}\right) ; \\
& \delta_{n}(c) \text { is the image of } \gamma_{n}(c) \text { in } H^{1}(K, E) l_{l^{n}} .
\end{aligned}
$$


This is the same as the construction in [1, Section 7.14.10], where we write here $\gamma_{n}(c), \delta_{n}(c)$ in place of $\gamma(c), \delta(c)$ to indicate their dependence on the integer $n$.

PROPOSITION 3.4.1

(a) The order of $\gamma_{n}(c)$ is equal to the order of $P_{c}\left(\bmod l^{n}\right)$ in $l^{n} E(K[c])$.

(b) The exponent $t$ of the order $l^{t}$ of $\delta_{n}(c)$ is the least integer $t$ such that

$$
l^{t}\left(P_{c}\left(\bmod l^{n}\right)\right) \in \frac{l^{n} E(K[c])+E(K)}{l^{n} E([c])} .
$$

Proof

Here $P_{c}\left(\bmod l^{n}\right)$ denotes the image of $P_{c} \in E(K[c])$ in $l^{n} E(K[c])$. These results on the orders of $\gamma_{n}(c)$ and $\delta_{n}(c)$ follow immediately from their definition and the commutative diagram (3.4.2).

\section{Part 4. Structure of the Tate-Shafarevich group and the Selmer group}

\subsection{Statement of the main theorems}

This section contains no proofs. The main theorems of this paper, Theorems 4.1.4, 4.1.8, 4.1.9, and 4.1.10, are finally proved in Sections 5.3-5.5.

\subsection{1.}

Throughout this section, we assume that

$E / F$ is an elliptic curve where $F$ is a global field of characteristic $p>0$;

$I$ is the ideal of $A$ which is the conductor of $E / F$ without the component at $\infty$;

$K$ is an imaginary quadratic extension field of $F$ with respect to $\infty$;

$l \in \mathcal{P}$ is a necessarily odd prime number in the set of prime numbers $\mathcal{P}$ (see Section 3.1);

$\epsilon= \pm 1$ is the sign of the functional equation of the $L$-function $L(E / F, s)$ of $E / F$;

$\tau$ is the element of order 2 of the Galois group $\operatorname{Gal}(K / F)$;

$P_{c} \in E(K[c])$ are the points defined in Section 3.4.8 over the ring class fields $K[c]$ for all divisors $c \in \Lambda(1)$ and where $P_{0}$ belongs to the group $E(K)$ of $K$-rational points;

$\alpha(c)$, for a divisor $c \in \Lambda(1)$, is the largest integer $n$ such that $c \in \Lambda(n)$ if $c \neq 0$ and such that $\alpha(0)=+\infty$ (see Remarks 3.2.4).

Assume that $E, K, F$ satisfy the following hypotheses (as in [1, Section 7.6.1]):

(a) $\infty$ is a place of $F$ with residue field equal to $k$;

(b) $E / F$ has split Tate multiplicative reduction at $\infty$ (see Section 1.7 or [1, Section 4.7]);

(c) $K$ is an (separable) imaginary quadratic field extension of $F$, with respect to $\infty$, such that all primes dividing the conductor $I$ split completely in $K$ and $K \neq F \otimes_{\mathbb{F}_{q}} \mathbb{F}_{q^{2}}$. 
These hypotheses (a), (b), and (c) are assumed for the rest of this paper.

\subsection{2.}

If $M$ is a $\operatorname{Gal}(K / F)$-module, then denote by $M^{+}$the submodule of $M$ on which $\tau$ acts by +1 and $M^{-}$is similarly the submodule on which $\tau$ acts by -1 .

\subsection{3.}

Let $G$ be a finite abelian $l$-group. The invariants of $G$ are the integers

$$
r_{1} \geq r_{2} \geq r_{3} \geq \cdots
$$

such that $G$ decomposes into elementary components

$$
G \cong \frac{\mathbb{Z}}{l^{r_{1}} \mathbb{Z}} \oplus \frac{\mathbb{Z}}{l^{r_{2}} \mathbb{Z}} \oplus \frac{\mathbb{Z}}{l^{r_{3}} \mathbb{Z}} \oplus \cdots
$$

The integers $r_{1}, r_{2}, r_{3}, \ldots$ are uniquely determined by $G$; the integers $l^{r_{1}}, l^{r_{2}}, l^{r_{3}}, \ldots$ are also sometimes called the invariants of $G$.

\subsection{4.}

As the elliptic curve $E$ is defined over $F$, the $l$-power torsion subgroup $\amalg(E / K)_{l^{\infty}}$ of the Tate-Shafarevich group $\amalg(E / K)$ of the elliptic curve $E \times_{F} K$ over $K$ decomposes into eigenspaces

$$
\amalg(E / K)_{l^{\infty}} \cong \amalg(E / K)_{l^{\infty}}^{+} \oplus \amalg(E / K)_{l^{\infty}}^{-}
$$

under the action of the element $\tau \in \operatorname{Gal}(K / F)$.

The Cassels pairing on $\amalg(E / K)$ is antisymmetric and respects this decomposition into eigenspaces. Furthermore, the Cassels pairing is nondegenerate if this Tate-Shafarevich group $\amalg(E / K)$ is finite; therefore, if $\amalg(E / K)$ is finite, then the invariants of the finite abelian $l$-group $\amalg(E / K)_{l^{\infty}}$ have even multiplicity.

\subsection{5.}

Under the hypothesis that the Tate-Shafarevich group $\amalg(E / K)_{l^{\infty}}$ is finite, let

$$
N_{1} \geq N_{3} \geq N_{5} \geq \cdots
$$

and

$$
N_{2} \geq N_{4} \geq N_{6} \geq \cdots
$$

be integers such that

$$
N_{1}, N_{1}, N_{3}, N_{3}, N_{5}, N_{5}, \ldots
$$

are the invariants of the finite abelian l-group $\amalg(E / K)_{l^{\infty}}^{\epsilon}$, which is the $\epsilon$ eigenspace, and

$$
N_{2}, N_{2}, N_{4}, N_{4}, N_{6}, N_{6}, \ldots
$$

are the invariants of the finite abelian l-group $\amalg(E / K)_{l \infty}^{-\epsilon}$, which is the $-\epsilon$ eigenspace. That is to say, putting $\nu(r)=(-1)^{r} \epsilon$ we have isomorphisms for $r=1$ 
or $r=2$,

$$
\amalg(E / K)_{l^{\infty}}^{-\nu(r)} \cong \bigoplus_{s \geq 0}\left(\mathbb{Z} / l^{N_{2 s+r}} \mathbb{Z}\right)^{2} .
$$

\section{DEFINITION 4.1.1}

Let $m \geq 1$ be an integer, and let $c \in \Lambda(m)$. Then the point $P_{c}$, defined in Section 3.4.8, belongs to $E(K[c])$ and the point $P_{0}$ belongs to $E(K)$.

(a) Write

$$
l^{m} \mid P_{c} \quad \text { if } P_{c} \in l^{m} E(K[c])
$$

and

$$
l^{m} \| P_{c} \quad \text { if } P_{c} \in l^{m} E(K[c]) \backslash l^{m+1} E(K[c]) .
$$

(b) For any integer $r \geq 0$, let $E(r)$ be the abelian group

$$
E(r)=\bigoplus_{c \in \Lambda^{r}(1)} A(c) E(K[c]),
$$

where the sum runs over all divisors $c$ of $\Lambda^{r}(1)$ and where $A(c)=l^{\alpha(c)}$. If $r>0$, then the group $E(r)$ is a direct sum of finite abelian $l$-groups of the form $E(K[c]) / l^{\alpha(c)} E(K[c])$. If $r=0$, then $E(0)$ is defined conventionally to be $E(K[0])$. A point $P_{c} \in E(K[c])$, where $c \in \Lambda^{r}(1)$, induces an element in $A_{(c)} E(K[c])$ and hence an element of $E(r)$ where all its components in $E(r)$ are zero except possibly for that in ${ }_{A(c)} E(K[c])$.

(c) Let $P(r)$ be the subgroup of $E(r)$ generated by the images in $E(r)$ of the points $P_{c}$ for all $c \in \Lambda^{r}(1)$.

Define $M_{r}$ for any $r \geq 0$ to be the largest integer $n \geq 0$ such that

$$
P(r) \subseteq l^{n} E(r) .
$$

If $P(r)=0$, then there is no such largest integer $M_{r}$ and we then put

$$
M_{r}=+\infty \text {. }
$$

\section{CONJECTURE 4.1.2}

For some $r \geq 0$ we have $M_{r}<+\infty$.

See [8, Conjecture A] and also [1, Conjecture 7.14.19] for an explanation of this conjecture.

\section{REMARKS 4.1.3}

(a) The order of $\gamma_{n}(c)$ is $l^{n-m}$ for all $n \geq m$ if and only if $l^{m} \| P_{c}$ by Proposition 3.4.1. If $M_{r}$ is finite, then there is a divisor $c \in \Lambda^{r}(1)$ such that $\gamma_{M_{r}+1}(c) \neq 0$ and $\gamma_{M_{r}}(c)=0$; furthermore, $\gamma_{M_{r}}(c)=0$ for any $c \in \Lambda^{r}(1)$ from Proposition 3.4.1(a).

(b) We have that $M_{0}<+\infty$ if and only if $P_{0}$ has infinite order in $E(K)$. This equivalence requires that $E(K)$ has no $l$-torsion, but this requirement holds by the 
restriction on the prime number $l$ given by Definition 3.1.2(b). See Lemma 5.1.1 below for details.

(c) We have that $\alpha(c)$ is finite if and only if $c \neq 0$ (see Remarks 3.2.4(b) and 3.2.4(c)). If $c \neq 0$, then write

$$
\begin{aligned}
& \operatorname{ord}_{l}\left(P_{c}\right) \\
& = \begin{cases}\max \left\{N: l^{N} \mid P_{c}\right\} & \text { if this maximum is }<\alpha(c), \\
+\infty & \text { if } \max \left\{N: l^{N} \mid P_{c}\right\} \text { is } \geq \alpha(c) .\end{cases}
\end{aligned}
$$

Write

$$
\operatorname{ord}_{l}\left(P_{0}\right)=\max \left\{N: l^{N} \mid P_{0}\right\},
$$

which is either an integer or $+\infty$. If $c \neq 0$, then we have $\operatorname{ord}_{l}\left(P_{c}\right)<+\infty$ if and only if $l^{\operatorname{ord}_{l}\left(P_{c}\right)} \| P_{c}$ and $\operatorname{ord}_{l}\left(P_{c}\right)<\alpha(c)$.

(d) By definition, for all integers $r \geq 0, M_{r}$ is given by

$$
M_{r}=\min \left\{\operatorname{ord}_{l}\left(P_{c}\right) \mid c \in \Lambda^{r}(1)\right\} .
$$

Note that $M_{r}<+\infty$ if and only if there is $c \in \Lambda^{r}(1)$ such that

$$
\operatorname{ord}_{l}\left(P_{c}\right)<\alpha(c)
$$

with an evident interpretation in the case where $r=0, c=0$, and $\alpha(0)=+\infty$. Furthermore, $M_{r}<+\infty$ implies that for this selection of $c \in \Lambda^{r}(1)$ we would have $l^{M_{r}} \| P_{c}, c \in \Lambda^{r}(\alpha(c))$, and $M_{r}<\alpha(c)$.

Alternatively, if $r>0$, then we have that $M_{r}<+\infty$ if and only if for some $c \in \Lambda^{r}(1)$ we have that

$$
c \in \Lambda^{r}(m+1) \backslash \Lambda^{r}(m+2)
$$

and

$$
\operatorname{ord}_{l}\left(P_{c}\right) \leq m
$$

\section{THEOREM 4.1.4}

Suppose that $P_{0}$ has infinite order in $E(K)$, the group of $K$-rational points of $E$. Let $l$ be a prime number in $\mathcal{P}$ coprime to the order of $\operatorname{Pic}(A)$, the Picard group of $A$. Then the Tate-Shafarevich group $\amalg(E / K)$ is finite and the invariants $N_{i}$, with multiplicity 2 , of the subgroup $\amalg(E / K)_{l \infty}$ are given by

$$
N_{i}=M_{i-1}-M_{i}, \quad \text { for } i \geq 1 .
$$

That is to say, we have the isomorphisms of eigenspaces

$$
\begin{aligned}
& \amalg(E / K)_{l \infty}^{\epsilon} \cong \prod_{\substack{i \text { even } \\
i \geq 0}}\left(\mathbb{Z} / l^{M_{i}-M_{i+1}} \mathbb{Z}\right)^{2}, \\
& \amalg(E / K)_{l^{\infty}}^{-\epsilon} \cong \prod_{\substack{i \text { odd } \\
i \geq 0}}\left(\mathbb{Z} / l^{M_{i}-M_{i+1}} \mathbb{Z}\right)^{2} .
\end{aligned}
$$


REMARKS 4.1.5

(a) The image of the Drinfeld-Heegner point $P_{0}$ in $E(K) / E(K)_{\text {tors }}$ is an eigenvector for $\tau$ and its eigenvalue is equal to $-\epsilon$ (by [1, Theorem 4.8.6, p. 98]; see also [1, Lemma 7.14.11, p. 388]) where $E(K)_{\text {tors }}$ is the torsion subgroup of $E(K)$.

(b) If the Drinfeld-Heegner point $P_{0}$ has infinite order in $E(K)$, then by [1, Theorem 7.7.5 and Remarks 7.7.6(3)] the Tate-Shafarevich group $\amalg(E / K)$ is finite and therefore the alternating Cassels pairing on $\amalg(E / K)$ is nondegenerate and by Sections 4.1 .4 and 4.1 .5 the invariants of the $\tau$-eigenspaces of $\amalg(E / K)_{l}$ have even multiplicity.

The next corollary is an immediate consequence of Theorem 4.1.4.

\section{COROLLARY 4.1.6}

Under the hypotheses of Theorem 4.1.4, the integers $M_{i}$ satisfy

$$
M_{i}-M_{i+1} \geq M_{i+2}-M_{i+3} \geq 0, \quad \text { for all } i \geq 0,
$$

and if $j$ is such that

$$
M_{j}=M_{j+1}=M_{j+2},
$$

then the sequence $M_{j}, M_{j+1}, M_{j+2}, M_{j+3}, \ldots$ is constant.

\section{DEFINITION 4.1.7}

Let

$$
G=G_{1} \times \cdots \times G_{r}
$$

be a direct product of finite cyclic groups $G_{i}$. The characters $\chi_{1}, \ldots, \chi_{r}$ of $G$ form a triangular basis for the dual $\widehat{G}$, relative to the product $G=\prod_{i} G_{i}$, if they generate $\widehat{G}$ and

$$
\chi_{i}\left(G_{j}\right)=0, \text { for all } j>i .
$$

\section{THEOREM 4.1.8}

Assume that $P_{0}$ has infinite order in $E(K)$, and assume that $l \in \mathcal{P}$ is a prime number coprime to the order of the Picard group $\operatorname{Pic}(A)$. Suppose that the direct product

$$
D=\prod_{i \geq 1} D_{i}
$$

is a maximal isotropic subgroup of $\amalg(E / K)_{l^{\infty}}$ for the Cassels pairing, and $D_{i}$ is a finite cyclic group of order $l^{N_{i}}$ for all $i \geq 1$, and we have direct products

$$
\begin{aligned}
D^{\epsilon} & =\prod_{i \text { odd }} D_{i}, \\
D^{-\epsilon} & =\prod_{i \text { even }} D_{i} .
\end{aligned}
$$


Then there are effective divisors $c_{1} \leq c_{2} \leq \cdots$ on $F$ such that $c_{i} \in \Lambda^{i}\left(M_{i-1}\right)$ for all $i \geq 1$ and the characters

$$
d \mapsto\left\langle d, \delta_{M_{i-1}}\left(c_{i}\right)\right\rangle_{\text {Cassels }} \text { for all } i
$$

form a triangular basis of characters of $D$ relative to the product $D=\prod_{i} D_{i}$.

\section{THEOREM 4.1.9}

Assume that $P_{0}$ has infinite order in $E(K)$, and assume that $l \in \mathcal{P}$ is coprime to the order of $\operatorname{Pic}(A)$. Let

$$
M_{\infty}=\min _{i \in \mathbf{N}} M_{i}
$$

Then the group $\mathbb{Z} P_{0}$ has finite index in $E(K)$ and the highest power of $l$ dividing the index $\left[E(K): \mathbf{Z} P_{0}\right]$ equals $l^{M_{0}}$; that is to say,

$$
\left|\left(E(K) / \mathbf{Z} P_{0}\right)_{l^{\infty}}\right|=l^{M_{0}} .
$$

Furthermore, we have that

$$
\left|\amalg(E / K)_{l \infty}\right|=l^{2\left(M_{0}-M_{\infty}\right)} .
$$

\section{THEOREM 4.1.10}

Assume that $P_{0}$ has infinite order in $E(K)$, and assume that $l \in \mathcal{P}$ is coprime to the order of $\operatorname{Pic}(A)$. Then for all integers $m \geq 0$, the natural surjection from the Selmer group to the Tate-Shafarevich group

$$
\pi_{m}: \operatorname{Sel}_{l^{m}}(E / K) \longrightarrow \amalg(E / K)_{l^{m}}
$$

splits and we have isomorphisms of eigenspaces

$$
\operatorname{Sel}_{l^{m}}(E / K)^{ \pm} \cong\left(l^{m} E(K)\right)^{ \pm} \oplus \amalg(E / K)_{l^{m}}^{ \pm} \quad \text { for all } m \geq 0 .
$$

Furthermore, we have isomorphisms for all $m \geq 0$

$$
\begin{aligned}
\left({ }_{l^{m}} E(K)\right)^{-\epsilon} \cong \mathbb{Z} / l^{m} \mathbb{Z}, \\
\left({ }_{l^{m}} E(K)\right)^{\epsilon} \cong 0 .
\end{aligned}
$$

The next corollary follows immediately from Theorems 4.1.4 and 4.1.10.

COROLLARY 4.1.11

Under the hypotheses of Theorem 4.1.10, put

$$
\widehat{N}_{i}=\min \left(m, M_{i-1}-M_{i}\right)
$$

for all $i$ and for all integers $m$. Then for all integers $m \geq 0$, the invariants of the

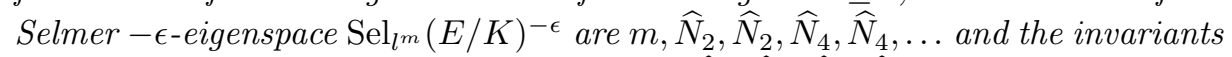
of the Selmer $\epsilon$-eigenspace $\operatorname{Sel}_{l^{m}}(E / K)^{\epsilon}$ are $\widehat{N}_{1}, \widehat{N}_{1}, \widehat{N}_{3}, \widehat{N}_{3}, \ldots$

\section{REMARK 4.1.12}

The main result on the finiteness of Tate-Shafarevich groups proved in the monograph [1, Theorem 7.6.5 and Theorem 7.7.5] is required for the proofs of the main 
results of this paper stated in this section; in particular, this paper does not provide a different proof of finiteness independent of the book [1]. This is also the principal reason why hypotheses (a), (b), and (c) of Section 4.1.1 are required.

\subsection{Cochains for the cohomology classes $\gamma_{n}(c), \delta_{n}(c)$}

The notation and hypotheses of Sections 4.1.1 and 4.1.2 hold in this section.

\section{LEMMA 4.2.1}

Let $c \in \Lambda(n)$.

(a) The cohomology class $\gamma_{n}(c)$ is represented by the cocycle

$$
\sigma \mapsto-\frac{(\sigma-1) P_{c}}{l^{n}}+\sigma \frac{P_{c}}{l^{n}}-\frac{P_{c}}{l^{n}}, \quad \operatorname{Gal}\left(K^{\mathrm{sep}} / K\right) \rightarrow E\left(K^{\mathrm{sep}}\right)_{l^{n}},
$$

where $\left[(\sigma-1) P_{c}\right] / l^{n}$ is the unique $l^{n}$-division point of $(\sigma-1) P_{c}$ in $E(K[c])$ and $P_{c} / l^{n}$ is a fixed $l^{n}$-division point of $P_{c}$.

(b) The cohomology class $\delta_{n}(c)$ is represented by the cocycle, where $\left[(\sigma-1) P_{c}\right] / l^{n}$ is the unique $l^{n}$-division point of $(\sigma-1) P_{c}$ in $E(K[c])$,

$$
\sigma \mapsto-\frac{(\sigma-1) P_{c}}{l^{n}}, \quad \operatorname{Gal}\left(K^{\mathrm{sep}} / K\right) \rightarrow E\left(K^{\mathrm{sep}}\right) .
$$

(c) Let $n \geq m$ be positive integers, and let $c \in \Lambda(n)$. Then we have that

$$
\begin{aligned}
& l^{m} \delta_{n}(c)=\delta_{n-m}(c), \\
& l^{m} \gamma_{n}(c)=\gamma_{n-m}(c) .
\end{aligned}
$$

Proof

(a), (b) These two formulae for cocycles representing the cohomology classes $\gamma_{n}(c)$ and $\delta_{n}(c)$ can be extracted from Step 2 of the proof of [1, Lemma 7.14.14]. We re-prove these formulae here.

From the diagram (3.4.2), the restriction homomorphism, where $\mathcal{G}_{c}=$ $\operatorname{Gal}(K[c] / K)$,

$$
H^{1}\left(K, E_{l^{n}}\left(K^{\mathrm{sep}}\right)\right) \rightarrow H^{1}\left(K[c], E_{l^{n}}\left(K^{\mathrm{sep}}\right)\right)^{\mathcal{G}_{c}}
$$

is an isomorphism as the prime number $l$ belongs to $\mathcal{P}$. The point $P_{c}$ belongs to $E(K[c])$.

Let $P_{c} / l^{n} \in E\left(K^{\mathrm{sep}}\right)$ be a fixed $l^{n}$ th division point of $P_{c}$; that is to say, $P_{c} / l^{n}$ is any point which satisfies $l^{n}\left(P_{c} / l^{n}\right)=P_{c}$. Then the cocycle

$$
\phi: g \mapsto g\left(\frac{P_{c}}{l^{n}}\right)-\frac{P_{c}}{l^{n}}, \quad \operatorname{Gal}\left(K^{\mathrm{sep}} / K[c]\right) \rightarrow E_{l^{n}}\left(K^{\mathrm{sep}}\right),
$$

represents a cohomology class in $H^{1}\left(K[c], E_{l^{n}}\left(K^{\mathrm{sep}}\right)\right)^{\mathcal{G}_{c}}$ which is the image of $P_{c}\left(\bmod l^{n}\right) \in\left(l^{n} E(K[c])\right)^{\mathcal{G}_{c}}$ under the coboundary map (see the diagram (3.4.2))

$$
\partial_{l^{n}}:\left(l^{n} E(K[c])\right)^{\mathcal{G}_{c}} \rightarrow H^{1}\left(K[c], E_{l^{n}}\left(K^{\mathrm{sep}}\right)\right)^{\mathcal{G}_{c}} .
$$


The inflation of $\phi$ to $\operatorname{Gal}\left(K^{\mathrm{sep}} / K\right)$ is given by the cocycle

$$
\phi^{\sharp}: \operatorname{Gal}\left(K^{\mathrm{sep}} / K\right) \rightarrow E\left(K^{\mathrm{sep}}\right), \quad g \mapsto g\left(\frac{P_{c}}{l^{n}}\right)-\frac{P_{c}}{l^{n}},
$$

which need not necessarily be annihilated by $l^{n}$.

For any element $g \in \operatorname{Gal}\left(K^{\mathrm{sep}} / K\right)$, denote by

$$
\frac{(g-1) P_{c}}{l^{n}}
$$

the unique $l^{n}$ th root of $(g-1) P_{c}$ in $E(K[c])$. This root exists because $P_{c}$ belongs to $\left(l^{n} E(K[c])^{\mathcal{G}_{c}}\right.$; furthermore, it is unique because $E(K[c])_{l^{\infty}}=0$ (by Definition 3.1.2(f), Proposition 1.10.1, and [1, Lemma 7.14.11(i)]).

The cochain

$$
\psi: \operatorname{Gal}\left(K^{\mathrm{sep}} / K\right) \rightarrow E(K[c]), \quad g \mapsto-\frac{(g-1) P_{c}}{l^{n}},
$$

is a cocycle whose restriction to the subgroup $\operatorname{Gal}\left(K^{\mathrm{sep}} / K[c]\right)$ is the zero cochain. But $\psi$ need not be annihilated by $l^{n}$. The cochain

$$
\phi^{\sharp}+\psi: \operatorname{Gal}\left(K^{\mathrm{sep}} / K\right) \rightarrow E\left(K^{\mathrm{sep}}\right), \quad g \mapsto g\left(\frac{P_{c}}{l^{n}}\right)-\frac{P_{c}}{l^{n}}-\frac{(g-1) P_{c}}{l^{n}},
$$

is a cocycle which is annihilated by $l^{n}$ and whose restriction to $\operatorname{Gal}\left(K^{\mathrm{sep}} / K[c]\right)$ is the cocycle

$$
\phi: \operatorname{Gal}\left(K^{\mathrm{sep}} / K[c]\right) \rightarrow E_{l^{n}}\left(K^{\mathrm{sep}}\right) .
$$

Hence, the cochain $\phi^{\sharp}+\psi$ is a cocycle

$$
\phi^{\sharp}+\psi: \operatorname{Gal}\left(K^{\mathrm{sep}} / K\right) \rightarrow E_{l^{n}}\left(K^{\mathrm{sep}}\right)
$$

and this cochain represents the cohomology class $\gamma_{n}(c)$ in $H^{1}\left(K, E_{l^{n}}\right)$. Therefore, the cohomology class $\delta_{n}(c)$ of $H^{1}(K[c] / K, E)_{l^{n}}$ is represented by the cocycle

$$
\psi: \operatorname{Gal}(K[c] / K) \rightarrow E\left(K^{\mathrm{sep}}\right), \quad g \mapsto-\frac{(g-1) P_{c}}{l^{n}},
$$

as required.

(c) This follows immediately from the explicit cocycle formulae of parts (a) and (b).

LEMMA 4.2.2

Denote by a superscript \pm 1 the eigenspaces under the action of the nontrivial element of $\operatorname{Gal}(K / F)$. If $c \in \Lambda^{r}(n)$, then we have

$$
\begin{aligned}
P_{c}\left(\bmod l^{n}\right) & \in\left(\left(l^{n} E(K[c])\right)^{\mathcal{G}_{c}}\right)^{-\nu(c)}, \\
\gamma_{n}(c) & \in H^{1}\left(K, E_{l^{n}}\right)^{-\nu(c)}, \\
\delta_{n}(c) & \in H^{1}(K[c] / K, E)_{l^{n}}^{-\nu(c)},
\end{aligned}
$$

where $\mathcal{G}_{c}=\operatorname{Gal}(K[c] / K)$,

$$
\nu(c)=(-1)^{r} \epsilon
$$


$r$ is the number of distinct prime divisors in the support of $c$, and $\epsilon$ is the sign in the functional equation of the L-function of $E / F$.

For the proof, see [1, Lemma 7.14.11]. Note that the set of prime numbers $\mathcal{P}$ is contained in the set of prime numbers denoted $\mathcal{P} \backslash \mathcal{F}$ in [1, Lemma 7.14.11].

\subsection{Points $P_{c}$ defined over local fields}

The notation and hypotheses of Section 4.1.1 hold in this section. The DrinfeldHeegner points $\left(a, I_{1}, c\right),\left(a, I_{1}, c, \pi\right)$ are defined in Sections 3.4.4 and 3.4.5.

\section{PROPOSITION 4.3.1}

Suppose that $c \in \Lambda(1)$ and $z \in \Lambda^{1}(1)$ is a prime divisor disjoint from the support of the divisor $c$. Let $y$ be the unique prime of $K$ lying over $z$, and let $K_{y}$ be the completion of $K$ at $y$. Then the point $\left(a, I_{1}, c\right) \in X_{0}^{\operatorname{Drin}}(K[c])$ is definable over $K_{y}$; that is to say,

$$
\left(a, I_{1}, c\right) \in X_{0}^{\text {Drin }}\left(K_{y}\right)
$$

Furthermore, we have that

$$
\left(a, I_{1}, c, \pi\right) \in E\left(K_{y}\right)
$$

is a point of the elliptic curve $E$ definable over $K_{y}$.

Proof

The prime $z$ is inert and unramified in $K / F$ by Definition 3.2 .1 and Remark 3.2.2(a). Furthermore, the elliptic curve $E / F$ has good reduction at $z$ by Remark 3.2.2(a). As $I$ is the conductor of $E / F$ without the component at $\infty$, by Section 4.1.1, we have that $z$ is disjoint from the support of $I$ and hence the curve $X_{0}^{\operatorname{Drin}}(I) / F$ also has good reduction $z$ where there may be several disjoint components in the closed fiber over $z$.

By [1, Theorem 4.6.19(ii)], because $z$ is inert and unramified in $K / F$, the reduction $\left(a, I_{1}, c\right)$ mod $z$ is defined over the quadratic extension field $\kappa(y)$ of $\kappa(z)$. That is to say, $\left(a, I_{1}, c\right) \bmod z$ is a point of the reduction at $z$ of $X_{0}^{\text {Drin }}(I)$ which is defined over $\kappa(y)$.

Let $F_{z}$ be the completion of $F$ at $z$. As $X_{0}^{\text {Drin }}(I)$ has good reduction at $z$, it follows that the point $\left(a, I_{1}, c\right)$ is defined over the field $K_{y}$ as this is the unique quadratic extension field of the local field $F_{z}$ which is unramified over $z$. It then immediately follows that $\left(a, I_{1}, c, \pi\right)$, which is the image of $\left(a, I_{1}, c\right)$ under the morphism $\pi: X_{0}^{\text {Drin }} \rightarrow E$ of $F$-schemes (see Sections 3.4.4, 3.4.5), is a point of the elliptic curve $E$ defined over $K_{y}$.

\section{PROPOSITION 4.3.2}

If $z \in \Lambda^{1}(1)$ is a prime divisor disjoint from the support of the divisor $c \in \Lambda(1)$ and $y$ is the prime of $K$ lying over $z$, then the image of $P_{c}$ in $l^{n} E\left(K_{y}\right)$ via Proposition 4.3.1 is uniquely determined by $P_{c}$. 
Proof

We have the isomorphism

$$
K[c] \otimes K_{y} \cong \prod_{i} K[c]_{x_{i}},
$$

where the $x_{i}$ 's are the places of $K[c]$ over the place $y$ of $K$ and $K[c]_{x_{i}}$ is the completion of $K[c]$ at $x_{i}$. The Galois group $\mathcal{G}_{c}=\operatorname{Gal}(K[c] / K)$ permutes transitively the places $x_{i}$ and the completions $K[c]_{x_{i}}$. We then have that

$$
{ }_{l^{n}} E\left(K[c] \otimes_{K} K_{y}\right) \cong \prod_{i} \iota^{n} E\left(K[c]_{x_{i}}\right) .
$$

The point $P_{c}$ belongs to $E(K[c])$ by construction. Hence, the point $P_{c}\left(\bmod l^{n}\right)$ of $l^{n} E(K[c])$ induces an element $\left(q_{1}, q_{2}, \ldots\right)$ of $l^{n} E\left(K[c] \otimes_{K} K_{y}\right)$ where

$$
\left(q_{1}, q_{2}, \ldots\right) \in \prod_{i} l^{n} E\left(K[c]_{x_{i}}\right)
$$

and

$$
q_{i} \in l_{l^{n}} E\left(K[c]_{x_{i}}\right) \text { for all } i .
$$

By Proposition 4.3.1, $P_{c}$ is definable over $K_{y}$; that is to say, $P_{c} \in E\left(K_{y}\right)$ and so we have $q_{i} \in l_{l^{n}} E\left(K_{y}\right)$ for all $i$. But $P_{c}\left(\bmod l^{n}\right) \in\left(l^{n} E(K[c])\right)^{\mathcal{G}_{c}}$ by Section 3.4.11 (see also Lemma 4.2.2). It follows that $\left(q_{1}, q_{2}, \ldots\right)$ is invariant under $\mathcal{G}_{c}$. But the elements $q_{i} \in l^{n} E\left(K_{y}\right)$ are permuted transitively by $\mathcal{G}_{c}$. Hence, the elements $q_{i}$ are all equal and $P_{c}\left(\bmod l^{n}\right)$ is the point $\left(q_{1}, q_{1}, \ldots\right) \in \prod_{i} l^{n} E\left([c]_{x_{i}}\right)$ which is in the image of the diagonal map ${ }_{l^{n}} E\left(K_{y}\right) \rightarrow \prod_{i} l^{n} E\left(K[c]_{x_{i}}\right)$. Hence, the components of the point $P_{c}\left(\bmod l^{n}\right)$ in $l^{n} E\left(K[c] \otimes_{K} K_{y}\right)$ are independent of the place $x_{i}$ and depend only on $P_{c}$ as required.

\subsection{The $\operatorname{map} \chi_{z}$}

The notation and hypotheses of Section 4.1.1 hold in this section.

\section{PROPOSITION 4.4.1}

Let $z \in \Lambda^{1}(n)$ be a prime divisor, and let $\mathcal{E}_{0} / \kappa(z)$ be the closed fiber of the Néron model of $E / F$ at the place $z$. Let $a_{z} \in \mathbb{Z}$ be the trace of the Frobenius at $z$ on the inertia invariant part of the Tate module of $E$ as in Section 3.2.4. Let $y$ be the unique place of $K$ over $z$. Let $c \in \Lambda(n)$ be a divisor whose support contains $z$, and put $c^{\prime}=c-z \in \Lambda(n)$ so that $c^{\prime}$ has support coprime to $z$.

(a) The endomorphism $\left|G\left(c / c^{\prime}\right)\right| \operatorname{Frob}(z)-a_{z}$ of the elliptic curve $\mathcal{E}_{0} / \kappa(z)$ annihilates the abelian group $\mathcal{E}_{0}(\kappa(y))$.

(b) The group homomorphism

$$
h: l^{n} \mathcal{E}_{0}(\kappa(y)) \rightarrow \mathcal{E}_{0}(\kappa(y))_{l^{n}}, \quad x \mapsto\left(\frac{\left|G\left(c / c^{\prime}\right)\right| \operatorname{Frob}(z)-a_{z}}{l^{n}}\right) x
$$

is an isomorphism which commutes with $\tau$. 


\section{Proof}

(a) As in Section 1.4, $G\left(c / c^{\prime}\right)$ denotes the Galois group $\operatorname{Gal}\left(K[c] / K\left[c^{\prime}\right]\right)$. The Frobenius $\operatorname{Frob}(z)$ acts on the Tate module of the elliptic curve $\mathcal{E}_{0} / \kappa(z)$ with characteristic polynomial

$$
X^{2}-X a_{z}+|\kappa(z)| \text {. }
$$

Writing $F$ for $\operatorname{Frob}(z)$, we have that $F^{2}-F a_{z}+|\kappa(z)|$ annihilates the abelian group $\mathcal{E}_{0}(\kappa(y))$. But $F^{2}$ is the identity automorphism on $\mathcal{E}_{0}(\kappa(y))$ as $\kappa(y) / \kappa(z)$ is a quadratic extension of finite fields. Hence, $F^{2}-F a_{z}+F^{2}|\kappa(z)|$ annihilates $\mathcal{E}_{0}(\kappa(y))$. That is to say, $F\left(F(1+|\kappa(z)|)-a_{z}\right)$ annihilates $\mathcal{E}_{0}(\kappa(y))$. As $F$ is an automorphism of $\mathcal{E}_{0}(\overline{\kappa(y)})$ we obtain that $F(1+|\kappa(z)|)-a_{z}$ annihilates $\mathcal{E}_{0}(\kappa(y))$.

As $K \neq F \otimes_{\mathbb{F}_{q}} \mathbb{F}_{q^{2}}$ (by the hypothesis (c) of Section 4.1.1) we have that the unit group $B^{*} / A^{*}$ is the trivial group. The Galois group

$$
G\left(c / c^{\prime}\right)=\operatorname{Gal}\left(K[c] / K\left[c^{\prime}\right]\right)
$$

therefore has order (see $[1,(2.3 .8),(2.3 .12)]$ )

$$
\left|G\left(c / c^{\prime}\right)\right|=|\kappa(z)|+1 \text {. }
$$

Hence the endomorphism $\left|G\left(c / c^{\prime}\right)\right| \operatorname{Frob}(z)-a_{z}$ of $\mathcal{E}_{0}$ annihilates the abelian group $\mathcal{E}_{0}(\kappa(y))$ as required.

(b) Let $\alpha, \beta \in \mathbb{C}$ be the complex roots of the characteristic polynomial of the Frobenius $\operatorname{Frob}(z)$ acting on the Tate module of $\mathcal{E}_{0} / \kappa(z)$

$$
X^{2}-X a_{z}+|\kappa(z)| \text {. }
$$

Then we have by the trace formula for the Frobenius automorphism, where $G\left(c / c^{\prime}\right)$ is cyclic of order $|\kappa(z)|+1$ as in the proof of part (a),

$$
\left|\mathcal{E}_{0}(\kappa(z))\right|=\left|G\left(c / c^{\prime}\right)\right|-\alpha-\beta=\left|G\left(c / c^{\prime}\right)\right|-a_{z}
$$

and

$$
\left|\mathcal{E}_{0}(\kappa(y))\right|=|\kappa(z)|^{2}+1-\alpha^{2}-\beta^{2}=\left(\left|G\left(c / c^{\prime}\right)\right|-a_{z}\right)\left(\left|G\left(c / c^{\prime}\right)\right|+a_{z}\right) .
$$

We then obtain the decomposition into eigenspaces under the action of the involution $\tau$, the nontrivial element of $\operatorname{Gal}(K / F)$,

$$
\mathcal{E}_{0}(\kappa(y)) \cong \mathcal{E}_{0}(\kappa(z)) \oplus \mathcal{E}_{0}(\kappa(y))^{-},
$$

where

$$
\left|\mathcal{E}_{0}(\kappa(y))^{\delta}\right|=\left|G\left(c / c^{\prime}\right)\right|-\delta a_{z} \quad \text { for } \delta= \pm 1 .
$$

Hence, the order of the $l^{\infty}$-torsion is given by

$$
\left|\mathcal{E}_{0}(\kappa(y))_{l^{\infty}}^{\delta}\right|=l^{s(\delta)},
$$

where $l^{s(\delta)}$ is the highest power of $l$ dividing $\delta\left|G\left(c / c^{\prime}\right)\right|-a_{z}$.

By Lemma 3.2.3(b), we have group isomorphisms for the $l^{n}$-torsion

$$
\mathcal{E}_{0}(\kappa(y))_{l^{n}}^{\delta} \cong \frac{\mathbb{Z}}{l^{n} \mathbb{Z}} \quad \text { for } \delta= \pm 1
$$


that is to say, these groups are cyclic of order $l^{n}$. It follows from this that there are isomorphisms for the $l^{\infty}$-torsion

$$
\mathcal{E}_{0}(\kappa(y))_{l^{\infty}}^{\delta} \cong \frac{\mathbb{Z}}{l^{s(\delta)} \mathbb{Z}} \quad \text { for } \delta= \pm 1 .
$$

We obtain that the $l^{\infty}$-torsion subgroups of $\mathcal{E}_{0}(\kappa(y))_{l^{\infty}}^{-}$and $\mathcal{E}_{0}(\kappa(y))_{l^{\infty}}^{+}$are both cyclic. Denoting by $|\cdot|_{l}$ the normalized $l$-adic absolute value on $\mathbb{Q}$ we have

$$
\left|\mathcal{E}_{0}(\kappa(y))_{l^{\infty}}^{\delta}\right|=|\delta| G\left(c / c^{\prime}\right)\left|-a_{z}\right|_{l}^{-1} \quad \text { for } \delta= \pm 1 .
$$

Let $g$ be the homomorphism

$$
g=\frac{\left|G\left(c / c^{\prime}\right)\right| \operatorname{Frob}(z)-a_{z}}{l^{n}}: \mathcal{E}_{0} \rightarrow \mathcal{E}_{0},
$$

where the integers $\left|G\left(c / c^{\prime}\right)\right|, a_{z}$ are both divisible by $l^{n}$ by Lemma 3.2.3(a). The homomorphism induced by $g$ on the $\kappa(y)$-rational points of $\mathcal{E}_{0}$

$$
g: \mathcal{E}_{0}(\kappa(y)) \rightarrow \mathcal{E}_{0}(\kappa(y))
$$

is annihilated by $l^{n}$ by part (a); furthermore, the subgroup $l^{n} \mathcal{E}_{0}(\kappa(y))$ of $\mathcal{E}_{0}(\kappa(y))$ belongs to the kernel of $g$ again by part (a). Hence, $g$ induces a homomorphism

$$
h:{ }_{l^{n}} \mathcal{E}_{0}(\kappa(y)) \rightarrow \mathcal{E}_{0}(\kappa(y))_{l^{n}} .
$$

On each eigencomponent $\left(l^{n} \mathcal{E}_{0}(\kappa(y))\right)^{\delta}$ under the action of $\tau$, the nontrivial element of $\operatorname{Gal}(K / F)$, and where $\delta= \pm 1$, the map $g$ is multiplication by the integer

$$
N(\delta)=\frac{\delta\left|G\left(c / c^{\prime}\right)\right|-a_{z}}{l^{n}}=\frac{\delta\left|\mathcal{E}_{0}(\kappa(y))^{\delta}\right|}{l^{n}} .
$$

It follows from this formula for $N(\delta)$ that the restriction of $h$ to each eigencomponent $\left(l^{n} \mathcal{E}_{0}(\kappa(y))\right)^{\delta}$ is an injection. As $\tau$ commutes with $h$, the homomorphism $h$ preserves the $\tau$-eigencomponents and therefore $h$ is an injection. As $l^{n} \mathcal{E}_{0}(\kappa(y))$ and $\mathcal{E}_{0}(\kappa(y))_{l^{n}}$ have the same number $l^{2 n}$ of elements it follows that $h$ is an isomorphism.

\section{PROPOSITION 4.4.2}

Let $z$ be a prime divisor of $F$ which belongs to $\Lambda^{1}(n)$. Let $y \in \Sigma_{K}$ be the unique place of $K$ lying over $z$. Assume that the prime number $l \in \mathcal{P}$ is coprime to the order of $\operatorname{Pic}(A)$, the Picard group of the ring A. Then there is a homomorphism

$$
\chi_{z}: l^{n} E\left(K_{y}\right) \rightarrow H^{1}\left(K_{y}, E_{l^{n}}\right)
$$

with the following properties.

(a) Let $x$ be a place of the ring class field $K[z]$ above the place $z$ of $K$. Then the image of $\chi_{z}$ is contained in the subgroup $H^{1}\left(K[z]_{x} / K_{y}, E_{l^{n}}\left(K[z]_{x}\right)\right)$.

(b) The homomorphism $\chi_{z}$ is injective.

(c) The composition of $\chi_{z}$ with the homomorphism, obtained from the inclusion of group schemes $E_{l^{n}} \subset E$,

$$
H^{1}\left(K_{y}, E_{l^{n}}\right) \rightarrow H^{1}\left(K_{y}, E\right)_{l^{n}}
$$


is an isomorphism

$$
l^{n} E\left(K_{y}\right) \cong H^{1}\left(K_{y}, E\right)_{l^{n}} .
$$

(d) For all divisors $c \in \Lambda(n)$ such that $z$ belongs to $\operatorname{Supp}(c)$, the cohomology class $\gamma_{n}(c)$ satisfies

$$
\gamma_{n}(c)_{y}=\chi_{z}\left(P_{c-z}\left(\bmod l^{n}\right)\right),
$$

where $P_{c-z}\left(\bmod l^{n}\right)$ is an element of $l^{n} E\left(K_{y}\right)$ (see Proposition 4.3.2) and $c-z$ has support coprime to $z$.

Proof

For all divisors $d \in \Lambda(n)$ with support coprime to $z$, by Propositions 4.3 .1 and 4.3.2 the point $P_{d} \in E(K[c])$ induces an element of $l^{n} E\left(K_{y}\right)$ which is uniquely determined by $P_{d}$ where $y$ is the place of $K$ over $z$ (see Remark 3.2.2(a)). Select a divisor $c \in \Lambda(n)$ with support containing $z$, and put

$$
c^{\prime}=c-z \in \Lambda(n)
$$

where $\operatorname{Supp}\left(c^{\prime}\right)$ is coprime to $z$.

Let $z^{\prime}$ be a prime divisor of $K\left[c^{\prime}\right]$ over the place $y$, which is the place of $K$ over $z$. The prime divisor $z^{\prime}$ is totally ramified in the field extension $K[c] / K\left[c^{\prime}\right]$; this follows via class field theory from the definition of the ring class field $K[c]$ (see Section 1.4; more details are given in $[1,(2.3 .13)]$ ). Let $z^{\times}$be the unique prime divisor of $K[c]$ lying over $z^{\prime}$.

The cohomology class $\delta_{n}(c)$ belongs to $H^{1}(K[c] / K, E(K[c]))_{l^{n}}$, which is contained via the inflation map in $H^{1}\left(K, E\left(K^{\text {sep }}\right)\right)_{l^{n}}$ (see diagram (3.4.2)).

Let $\mathcal{E}_{0} / \kappa(z)$ be the closed fiber above $z$ of the Néron model of $E / F$. We then define a composite isomorphism $\Phi$ as follows where the maps $i, h, j$ are explained below:

$$
\Phi: l^{n} E\left(K_{y}\right) \stackrel{i}{\longrightarrow} l^{n} \mathcal{E}_{0}(\kappa(y)) \stackrel{h}{\longrightarrow} \mathcal{E}_{0}(\kappa(y))_{l^{n}} \stackrel{j}{\longrightarrow} E\left(K_{y}\right)_{l^{n}} .
$$

Here $i: l^{n} E\left(K_{y}\right) \longrightarrow l^{n} \mathcal{E}_{0}(\kappa(y))$ is the isomorphism obtained from the surjective homomorphism $E\left(K_{y}\right) \longrightarrow \mathcal{E}_{0}(\kappa(y))$ of reduction at $z$ whose kernel is a pro-p-group. The map $h: l^{n} \mathcal{E}_{0}(\kappa(y)) \longrightarrow \mathcal{E}_{0}(\kappa(y))_{l^{n}}$ is the isomorphism of Proposition 4.4.1(b). The map $j: \mathcal{E}_{0}(\kappa(y))_{l^{n}} \longrightarrow E\left(K_{y}\right)_{l^{n}}$ is the isomorphism obtained from reduction modulo $y$; the map $j$ is an isomorphism because $E$ has good reduction at $y$ and the prime number $l$ is distinct from the characteristic of $F$. The map $\Phi$ is an isomorphism as $i, h, j$ are isomorphisms.

Let $x^{\prime}$ be a prime of $K[0]$ lying over $y$, and let $x$ be the unique prime of $K[z]$ over $x^{\prime}$, where $y$ is the prime of $K$ over $z$. The prime $x$ over $x^{\prime}$ is uniquely determined by $x^{\prime}$ because $K[z] / K[0]$ is totally ramified at $x^{\prime}$ (see Section 1.4). The restriction of elements of the Galois group $G\left(c / c^{\prime}\right)$ to the fields $K[z]$ and $K[z]_{x}$ induces isomorphisms

$$
\operatorname{Gal}\left(K[z]_{x} / K[0]_{x^{\prime}}\right) \cong G(z / 0) \cong G(c / c-z),
$$


where these groups are cyclic of order $|\kappa(z)|+1$ (by [1, (2.3.12), p. 20], and as $B^{*} / A^{*}$ is the trivial group by hypothesis (c) of Section 4.1.1). Put

$$
G=\operatorname{Gal}\left(K[z]_{x} / K[0]_{x^{\prime}}\right) .
$$

We have the exact sequence of Galois groups

$$
0 \rightarrow G \rightarrow \operatorname{Gal}\left(K[z]_{x} / K_{y}\right) \rightarrow \operatorname{Gal}\left(K[0]_{x^{\prime}} / K_{y}\right) \rightarrow 0 .
$$

As the field extension $K[z] / K[0]$ is totally ramified at the place $x^{\prime}$ of $K[0]$ over $z$, the restriction homomorphism of $G(c / 0)$ to $K[0]_{x^{\prime}}$ gives the isomorphism

$$
G \cong G(z / 0)
$$

of (4.4.2).

As the field extension $K[z]_{x} / K[0]_{x^{\prime}}$ is totally ramified, we have that the group $G$ acts trivially on $E_{l^{n}}\left(K[z]_{x}\right)$ and that

$$
E_{l^{n}}\left(K[z]_{x}\right)=E_{l^{n}}\left(K[0]_{x^{\prime}}\right) .
$$

Hence we have an isomorphism

$$
H^{1}\left(G, E_{l^{n}}\left(E\left(K[z]_{x}\right)\right)\right) \cong \operatorname{Hom}\left(G, E_{l^{n}}\left(K[0]_{x^{\prime}}\right)\right) .
$$

We have already fixed in Section 3.4.6 a generator $\sigma_{z}$ of $G\left(c / c^{\prime}\right)$. Let $\sigma \in G$ be the generator induced by $\sigma_{z}$ under the isomorphism $G\left(c / c^{\prime}\right) \cong G$ of (4.4.2).

For any $P \in l_{l^{n}} E\left(K_{y}\right)$, define a homomorphism

$$
f_{P}: G \rightarrow E_{l^{n}}\left(K_{y}\right)
$$

as follows. Put

$$
f_{P}(\sigma)=\Phi(P)
$$

where $\sigma$ is the chosen generator of the cyclic group $G$. As $\Phi(P)$ is a point of $E_{l^{n}}\left(K_{y}\right)$ and as the order of the cyclic group $G$ is equal to $|\kappa(z)|+1$, which is divisible by $l^{n}$, the homomorphism $f_{P}$ is well defined. Hence, $f_{P}$ defines a cohomology class in $H^{1}\left(G, E_{l^{n}}\left(K_{y}\right)\right)$ where $G$ acts trivially on $E_{l^{n}}\left(K_{y}\right)$.

As $\Phi: l^{n} E\left(K_{y}\right) \rightarrow E\left(K_{y}\right)_{l^{n}}$ is an isomorphism (see (4.4.1)) and $G$ is cyclic of order divisible by $l^{n}$, this map $P \mapsto f_{P}$ defines a group isomorphism

$$
f: l^{n} E\left(K_{y}\right) \stackrel{\cong}{\longrightarrow} H^{1}\left(G, E_{l^{n}}\left(K_{y}\right)\right), \quad P \mapsto f_{P} .
$$

We have the Hochschild-Serre spectral sequence

$$
E_{2}^{i, j} \Rightarrow H^{i+j}\left(K[c]_{z^{\times}} / K_{y}, E_{l^{n}}\left(K[c]_{z^{\times}}\right)\right),
$$

where we write

$$
G_{z^{\times} / z^{\prime}}=\operatorname{Gal}\left(K[c]_{z^{\times}} / K\left[c^{\prime}\right]_{z^{\prime}}\right)
$$

and

$$
E_{2}^{i, j}=H^{i}\left(K\left[c^{\prime}\right]_{z^{\prime}} / K_{y}, H^{j}\left(G_{z^{\times} / z^{\prime}}, E_{l^{n}}\left(K[c]_{z^{\times}}\right)\right)\right) .
$$


The short exact sequence of low-degree terms attached to this spectral sequence in part takes the form

$$
0 \rightarrow E_{2}^{1,0} \rightarrow H^{1}\left(K[c]_{z^{\times}} / K_{y}, E_{l^{n}}\left(K[c]_{z^{\times}}\right)\right) \rightarrow E_{2}^{0,1} \rightarrow E_{2}^{2,0} .
$$

As the order of $\operatorname{Pic}(A)$ is coprime to $l$ we have that the order of the place $y$ above $z$ in $\operatorname{Pic}(B)$ is coprime to $l$, as $y$ is the unique place of $K$ over $z$. It follows that the degree of the field extension $K\left[c^{\prime}\right]_{z^{\prime}} / K_{y}$, which is equal to the degree of the residue field extensions, is coprime to $l$. Hence, we have

$$
H^{i}\left(K\left[c^{\prime}\right]_{z^{\prime}} / K_{y}, H^{j}\left(G_{z^{\times} / z^{\prime}}, E_{l^{n}}\left(K[c]_{z^{\times}}\right)\right)\right) \cong 0 \text { for all } i \geq 1
$$

as the group $H^{j}\left(G_{z^{\times} / z^{\prime}}, E_{l^{n}}\left(K[c]_{z^{\times}}\right)\right)$is $l$-power torsion for all $i$. The above short exact sequence of low-degree terms then becomes an isomorphism

$$
\begin{aligned}
& H^{1}\left(K[c]_{z^{\times}} / K_{y}, E_{l^{n}}\left(K[c]_{z^{\times}}\right)\right) \\
& \quad \cong H^{0}\left(K\left[c^{\prime}\right]_{z^{\prime}} / K_{y}, H^{1}\left(G_{z^{\times} / z^{\prime}}, E_{l^{n}}\left(K[c]_{z^{\times}}\right)\right)\right) .
\end{aligned}
$$

As the field extension $K[c]_{z^{\times}} / K\left[c^{\prime}\right]_{z^{\prime}}$ is totally ramified, we have $E_{l^{n}}\left(K[c]_{z^{\times}}\right)=E_{l^{n}}\left(K\left[c^{\prime}\right]_{z^{\prime}}\right)$ and the group $G_{z^{\times} / z^{\prime}}$ acts trivially on $E_{l^{n}}\left(K[c]_{z^{\times}}\right)$. This last isomorphism of (4.4.6) then becomes the isomorphism

$$
H^{1}\left(K[c]_{z^{\times}} / K_{y}, E_{l^{n}}\left(K[c]_{z^{\times}}\right)\right) \cong H^{0}\left(K\left[c^{\prime}\right]_{z^{\prime}} / K_{y}, \operatorname{Hom}\left(G_{z^{\times} / z^{\prime}}, E_{l^{n}}\left(K\left[c^{\prime}\right]_{z^{\prime}}\right)\right)\right),
$$

where $G_{z^{\times}} / z^{\prime}$ acts trivially on $E_{l^{n}}\left(K\left[c^{\prime}\right]_{z^{\prime}}\right)$. This then provides the isomorphism

$$
H^{0}\left(K\left[c^{\prime}\right]_{z^{\prime}} / K_{y}, H^{1}\left(G_{z^{\times} / z^{\prime}}, E_{l^{n}}\left(K[c]_{z^{\times}}\right)\right)\right) \cong \operatorname{Hom}\left(G_{z^{\times} / z^{\prime}}, E_{l^{n}}\left(K_{y}\right)\right) .
$$

This isomorphism combined with the isomorphism of (4.4.6) gives the isomorphism

$$
H^{1}\left(K[c]_{z^{\times}} / K_{y}, E_{l^{n}}\left(K[c]_{z^{\times}}\right)\right) \cong H^{1}\left(G_{z^{\times} / z^{\prime}}, E_{l^{n}}\left(K_{y}\right)\right),
$$

where $G_{z^{\times} / z^{\prime}}$ acts trivially on $E_{l^{n}}\left(K_{y}\right)$.

Now, take $c=z$ in the isomorphism of (4.4.7) where $x^{\prime}$ is a place of $K[0]$ over $y$ and $x$ is the unique place of $K[z]$ over $x^{\prime}$; we have that the composition of this isomorphism (4.4.7) with the isomorphism $f$ of (4.4.5) gives the isomorphism

$$
\phi: l^{n} E\left(K_{y}\right) \stackrel{\cong}{\longrightarrow} H^{1}\left(K[z]_{x} / K_{y}, E_{l^{n}}\left(K[z]_{x}\right)\right) .
$$

The inflation map from $\operatorname{Gal}\left(K[z]_{x} / K_{y}\right)$ to $\operatorname{Gal}\left(K_{y}^{\text {sep }} / K_{y}\right)$ is an injective homomorphism

$$
H^{1}\left(K[z]_{x} / K_{y}, E_{l^{n}}\left(K[z]_{x}\right)\right) \stackrel{\inf }{\longrightarrow} H^{1}\left(K_{y}, E_{l^{n}}\left(K_{y}^{\mathrm{sep}}\right)\right) .
$$

The composite of this injective inflation map of (4.4.9) with the isomorphism $\phi$ of (4.4.8) is then defined to be the injective homomorphism $\chi_{z}$

$$
\begin{aligned}
\chi_{z}: l^{n} E\left(K_{y}\right) & \rightarrow H^{1}\left(K_{y}, E_{l^{n}}\right), \\
P & \mapsto\{\sigma \mapsto \Phi(P)\} .
\end{aligned}
$$

Here $\{\sigma \mapsto \Phi(P)\}$ denotes the cohomology class $f_{P}$ of (4.4.4) and (4.4.5) defined by $\sigma \mapsto \Phi(P)$ where $\sigma \in G$ is the chosen generator of $G$; this $f_{P}$ then 
defines a cohomology class in $H^{1}\left(K[z]_{x} / K_{y}, E_{l^{n}}\left(K[z]_{x}\right)\right)$ by the isomorphism of (4.4.7) for $c=z$. By inflation, this $f_{P}$ gives a cohomology class of $\operatorname{Gal}\left(K_{y}^{\text {sep }} / K_{y}\right)$ and hence an element of the cohomology group $H^{1}\left(K_{y}, E_{l^{n}}\right)$ and this defines the homomorphism $\chi_{z}$. This map $\chi_{z}$ of (4.4.10) is injective by construction.

To prove property (a), by construction the homomorphism

$$
\chi_{z}: l^{n} E\left(K_{y}\right) \longrightarrow H^{1}\left(K_{y}, E_{l^{n}}\right)
$$

takes a point $P \in l^{n} E\left(K_{y}\right)$ to an element $f_{P}$ of $H^{1}\left(G, E_{l^{n}}\left(K[z]_{x}\right)\right)$ which is inflated to an element of $H^{1}\left(K_{y}, E_{l^{n}}\left(K^{\text {sep }}\right)\right)$, and this proves property (a) of the map $\chi_{z}$.

To prove property (b), the map

$$
\chi_{z}: l^{n} E\left(K_{y}\right) \rightarrow H^{1}\left(K_{y}, E_{l^{n}}\right), \quad P \mapsto\{\sigma \mapsto \Phi(P)\}
$$

is injective by construction, as already noted.

For property (c), we have by definition that the map $\chi_{z}$ of (4.4.10) is the composition of the isomorphism $\phi$ of (4.4.8) with the inflation map of (4.4.9). That is to say, the map $\chi_{z}$ factors as

$$
l^{n} E\left(K_{y}\right) \stackrel{\phi}{\longrightarrow} H^{1}\left(K[z]_{x} / K_{y}, E_{l^{n}}\left(K[z]_{x}\right)\right) \stackrel{\inf }{\longrightarrow} H^{1}\left(K_{y}, E_{l^{n}}\left(K_{y}^{\mathrm{sep}}\right)\right),
$$

where $\phi$ is an isomorphism and inf is an injection.

We have an exact sequence obtained from the inclusion of group schemes $E_{l^{n}} \subset E$,

$$
0 \longrightarrow l^{n} E\left(K_{y}\right) \stackrel{\partial_{l^{n}}}{\longrightarrow} H^{1}\left(K_{y}, E_{l^{n}}\right) \stackrel{\psi}{\longrightarrow} H^{1}\left(K_{y}, E\right)_{l^{n}} .
$$

The morphism of multiplication by $l^{n}$ on the Néron model of $E$ over the ring of valuation integers of $F$ at the place $z$ is étale; therefore, the image of $\partial_{l^{n}}$ consists of unramified cohomology classes, and more precisely, the image of $\partial_{l^{n}}$ belongs to the subgroup $H^{1}\left(K_{y}^{\mathrm{nr}} / K_{y}, E_{l^{n}}\right)$ of $H^{1}\left(K_{y}, E_{l^{n}}\right)$ where $K_{y}^{n r}$ is the maximal separable unramified extension of $K_{y}$. The intersection of $H^{1}\left(K_{y}^{\mathrm{nr}} / K_{y}, E_{l^{n}}\right)$ with $H^{1}\left(K[z]_{x} / K_{y}, E_{l^{n}}\left(K[z]_{x}\right)\right)$, which are both subgroups of $H^{1}\left(K_{y}, E_{l^{n}}\right)$, is therefore contained in $H^{1}\left(K[0]_{x^{\prime}} / K_{y}, E_{l^{n}}\left(K[0]_{x^{\prime}}\right)\right)$; this follows by considering representative cocycles and because the field extension $K[z]_{x} / K[0]_{x^{\prime}}$ is totally ramified and $K[0]_{x^{\prime}} / K_{y}$ is unramified. But the order of the Picard group $\operatorname{Pic}(A)$ is coprime to $l$ by hypothesis; therefore, the order of the place $y$ over $z$ in $\operatorname{Pic}(B)$ is coprime to $l$ as $y$ is the unique place of $K$ over $z$. It follows that the degree of the field extension $K[0]_{x^{\prime}} / K_{y}$ is coprime to $l$, and therefore we have that

$$
H^{1}\left(K[0]_{x^{\prime}} / K_{y}, E_{l^{n}}\left(K[0]_{x^{\prime}}\right)\right)=0 .
$$

It follows that the composition of the injective inflation map

$$
H^{1}\left(K[z]_{x} / K_{y}, E_{l^{n}}\left(K[z]_{x}\right)\right) \stackrel{\text { inf }}{\longrightarrow} H^{1}\left(K_{y}, E_{l^{n}}\left(K_{y}^{\text {sep }}\right)\right)
$$

with $\psi: H^{1}\left(K_{y}, E_{l^{n}}\right) \rightarrow H^{1}\left(K_{y}, E\right)_{l^{n}}$ is an injection

$$
H^{1}\left(K[z]_{x} / K_{y}, E_{l^{n}}\left(K[z]_{x}\right)\right) \longrightarrow H^{1}\left(K_{y}, E\right)_{l^{n}} .
$$


By arithmetic flat local duality, there is an isomorphism of discrete groups

$$
H^{1}\left(K_{y}, E\right) \cong \operatorname{Hom}\left(E\left(K_{y}\right), \mathbb{Q} / \mathbb{Z}\right) .
$$

For the proof of this using flat cohomology and local class field theory, see [10, Chapter III, Theorem 7.8]; we only require the prime-to- $p$ version of this duality (4.4.13), whose simpler proof is explained in [10, Chapter I, Remark 3.6]. From (4.4.13) we obtain the isomorphism of discrete groups

$$
H^{1}\left(K_{y}, E\right)_{l^{n}} \cong \operatorname{Hom}\left(l^{n} E\left(K_{y}\right), \mathbb{Z} / l^{n} \mathbb{Z}\right) .
$$

It follows from (4.4.14) that $H^{1}\left(K_{y}, E\right)_{l^{n}}$ and $l^{n} E\left(K_{y}\right)$ have the same number of elements. As $E_{l^{n}}\left(K_{y}\right)$ is isomorphic to $l^{n} E\left(K_{y}\right)$ by the isomorphism $\Phi$ of (4.4.1), we have that $H^{1}\left(K_{y}, E\right)_{l^{n}}$ and $E_{l^{n}}\left(K_{y}\right)$ have the same number of elements.

From (4.4.7) for $c=z$, we have the isomorphism, where $G$ is cyclic of order divisible by $l^{n}$ and which acts trivially on $E_{l^{n}}\left(K_{y}\right)$

$$
H^{1}\left(K[z]_{x} / K_{y}, E_{l^{n}}\left(K[z]_{x}\right)\right) \cong H^{1}\left(G, E_{l^{n}}\left(K_{y}\right)\right) \cong E_{l^{n}}\left(K_{y}\right) .
$$

It now follows that the finite groups $H^{1}\left(K[z]_{x} / K_{y}, E_{l^{n}}\left(K[z]_{x}\right)\right), E_{l^{n}}\left(K_{y}\right)$, and $H^{1}\left(K_{y}, E\right)_{l^{n}}$ have the same number of elements and hence the injective homomorphism of (4.4.12)

$$
H^{1}\left(K[z]_{x} / K_{y}, E_{l^{n}}\left(K[z]_{x}\right)\right) \longrightarrow H^{1}\left(K_{y}, E\right)_{l^{n}}
$$

is an isomorphism. It follows that the natural homomorphism, obtained from the inclusion of group schemes $E_{l^{n}} \subset E$,

$$
H^{1}\left(K_{y}, E_{l^{n}}\right) \stackrel{\psi}{\longrightarrow} H^{1}\left(K_{y}, E\right)_{l^{n}}
$$

composed with $\chi_{z}$, where $\chi_{z}$ factors through the group $H^{1}\left(K[z]_{x} / K_{y}, E_{l^{n}}\left(K[z]_{x}\right)\right)$ as in (4.4.11),

$$
l^{n} E\left(K_{y}\right) \stackrel{\chi_{z}}{\longrightarrow} H^{1}\left(K_{y}, E_{l^{n}}\right) \stackrel{\psi}{\longrightarrow} H^{1}\left(K_{y}, E\right)_{l^{n}}
$$

is an isomorphism

$$
l^{n} E\left(K_{y}\right) \cong H^{1}\left(K_{y}, E\right)_{l^{n}} .
$$

This proves property (c).

It only remains to prove property (d). For all divisors $d \in \Lambda(n)$, as in Section 3.4.9 we shall write $P_{d}\left(\bmod l^{n}\right)$ for the image of $P_{d} \in E(K[d])$ in $l^{n} E(K[d])$.

From Lemma 4.2.1, we have that the cohomology class $\gamma_{n}(c)$ in $H^{1}\left(K, E_{l^{n}}\right)$ is represented by the cocycle

$$
\Gamma_{n}(c): \sigma \mapsto-\frac{(\sigma-1) P_{c}}{l^{n}}+\sigma \frac{P_{c}}{l^{n}}-\frac{P_{c}}{l^{n}}, \quad \operatorname{Gal}\left(K^{\mathrm{sep}} / K\right) \rightarrow E\left(K^{\mathrm{sep}}\right) l^{n},
$$

where $\left[(\sigma-1) P_{c}\right] / l^{n}$ is the unique $l^{n}$-division point of $(\sigma-1) P_{c}$ in $E(K[c])$ and $P_{c} / l^{n}$ is a fixed $l^{n}$-division point of $P_{c}$, and the cohomology class $\delta_{n}(c)$ in $H^{1}(K, E)_{l^{n}}$ is represented by the cocycle

$$
\Delta_{n}(c): \sigma \mapsto-\frac{(\sigma-1) P_{c}}{l^{n}}, \quad \operatorname{Gal}\left(K^{\mathrm{sep}} / K\right) \rightarrow E\left(K^{\mathrm{sep}}\right) .
$$


The cohomology class $\delta_{n}(c)_{y} \in H^{1}\left(K_{y}, E\left(K_{y}^{\text {sep }}\right)\right)$ is the restriction at $y$ of the class $\delta_{n}(c) \in H^{1}\left(K, E\left(K^{\mathrm{sep}}\right)\right)$. Let $Q \in E(K[c])$ be the element given by

$$
Q=-\frac{\left(\sigma_{z}-1\right) P_{c}}{l^{n}}
$$

where $\sigma_{z}$ is the fixed generator of the cyclic group $G\left(c / c^{\prime}\right)$.

The definition of $P_{c}$ (as in [1, Section 7.14.10, pp. 386-387] and Section 3.4.8 above) is the following. Let $c=\sum_{i=1}^{r} z_{i}$ be the decomposition of $c$ as a sum of distinct prime divisors, where we write $z=z_{1}$. Then we have that

$$
P_{c}=\sum_{s \in \mathcal{S}} s D_{c} y_{c}
$$

where

$$
y_{c}=\left(0, I_{1}, c, \pi\right) \in E(K[c])
$$

as in Section 3.4.6 and where $\mathcal{S}$ is a set of coset representatives for $G(c / 0)$ in $\operatorname{Gal}(K[c] / K)$ and $D_{c}$ is the Kolyvagin element of Section 3.4.8.

We have an exact sequence of abelian groups

$$
0 \rightarrow G\left(c / c^{\prime}\right) \rightarrow G(c / 0) \rightarrow G\left(c^{\prime} / 0\right) \rightarrow 0 .
$$

We obtain a corresponding decomposition in the group algebra $\mathbb{Z}[G(c / 0)]$ of the Kolyvagin element $D_{c}$

$$
D_{c}=D_{c-z} D_{z}
$$

We have already selected in Section 3.4 .6 a generator $\sigma_{z}$ of the cyclic group $G(c / c-z)$. We may then define a map of sets

$$
h_{z}: G(c / c-z) \rightarrow \mathbb{Z}
$$

by

$$
\sigma_{z}^{-s} \mapsto-s, \quad \text { for } s=0,1, \ldots,|G(c / c-z)|-1 .
$$

The Kolyvagin element of $h_{z}$ is then, as in Section 3.4.7,

$$
D_{z}=-\sum_{r=1}^{|G(c / c-z)|-1} r \sigma_{z}^{r} .
$$

We have that

$$
\left(\sigma_{z}-1\right) D_{z}=-|G(c / c-z)|+e_{G(c / c-z)},
$$

where $e_{G(c / c-z)}$ is the element of the group algebra $\mathbb{Z}[G(c / c-z)]$ given by

$$
e_{G(c / c-z)}=\sum_{g \in G(c / c-z)} g .
$$

The element $P_{c} \in E(K[c])$ may then be written as

$$
P_{c}=\sum_{s \in \mathcal{S}} s D_{c-z} D_{z} y_{c},
$$

where we write $y_{c}=\left(0, I_{1}, c, \pi\right)$ as in Section 3.4.6 and (4.4.18). 
We have that (see [1, (4.8.3), Table 4.8.5])

$$
\frac{\left|O_{c^{\prime}}^{*}\right|}{\left|A^{*}\right|} \operatorname{Tr}_{K[c] / K\left[c^{\prime}\right]} y_{c}=a_{z} y_{c^{\prime}}
$$

where $a_{z} \in \mathbb{Z}$ is as in Proposition 4.4.1 and Section 3.2.4 and where $y_{c^{\prime}}=$ $\left(0, I_{1}, c^{\prime}, \pi\right)$. By definition $Q=-\left(\left(\sigma_{z}-1\right) P_{c}\right) / l^{n}$ (see $\left.(4.4 .17)\right)$; hence, we have from (4.4.19) that

$$
\begin{aligned}
Q & =-\sum_{s \in \mathcal{S}} s D_{c-z}\left(\frac{\left(\sigma_{z}-1\right) D_{z}}{l^{n}}\right) y_{c} \\
& =\sum_{s \in \mathcal{S}} s D_{c-z}\left(\frac{\left|G\left(c / c^{\prime}\right)\right|}{l^{n}} y_{c}-\frac{\left|A^{*}\right|}{\left|O_{c^{\prime}}^{*}\right|} \frac{a_{z}}{l^{n}} y_{c^{\prime}}\right) .
\end{aligned}
$$

As $K$ is not obtained from $F$ by ground field extension (hypothesis (c) of Section 4.1.1), we have $A^{*}=O_{c^{\prime}}^{*}$; hence we obtain that

$$
Q=\sum_{s \in \mathcal{S}} s D_{c-z}\left(\frac{\left|G\left(c / c^{\prime}\right)\right|}{l^{n}} y_{c}-\frac{a_{z}}{l^{n}} y_{c^{\prime}}\right) .
$$

As at the beginning of this proof, let $y$ be the unique place of $K$ over $z$, let $z^{\prime}$ be a prime of $K\left[c^{\prime}\right]$ over the place $y$ of $K$, and let $z^{\times}$be the place of $K[c]$ over the prime $z^{\prime}$ of $K\left[c^{\prime}\right]$ where the field extension $K[c] / K\left[c^{\prime}\right]$ is totally ramified at $z^{\prime}$. Also $\mathcal{E}_{0} / \kappa(z)$ denotes the closed fiber above $z$ of the Néron model of $E / F$.

We write $Q_{0}$ for the image of $Q$ modulo $z^{\times}$in $\mathcal{E}_{0}\left(\kappa\left(z^{\times}\right)\right)$by passage to the residue field $\kappa\left(z^{\times}\right)$. From the isomorphism (4.4.7), where $\delta_{n}(c)_{y}$ belongs to $H^{1}\left(K[c]_{z^{\times}} / K_{y}, E_{l^{n}}\left(K[c]_{z^{\times}}\right)\right)$, we have that the reduction of $\delta_{n}(c)_{y}$ at $y$ belongs to $\operatorname{Hom}\left(G\left(c / c^{\prime}\right), \mathcal{E}_{0}(\kappa(y))\right)_{l^{n}}$ and is given by the cocycle (see (4.4.16))

$$
g \mapsto-\frac{(g-1) P_{c}}{l^{n}}\left(\bmod z^{\times}\right), \quad \operatorname{Gal}\left(K[c]_{z^{\times}} / K\left[c^{\prime}\right]_{z^{\prime}}\right) \cong G\left(c / c^{\prime}\right) \rightarrow \mathcal{E}_{0}(\kappa(y)) .
$$

We have that $-\left[(g-1) P_{c}\right] / l^{n}$ modulo $z^{\times}$belongs to the $l^{n}$-torsion subgroup $\mathcal{E}_{0}(\kappa(y))_{l^{n}}$ rational over $\kappa(y)$. Hence, the point $Q_{0}$, the reduction of $Q$ modulo $z^{\times}$, belongs to $\mathcal{E}_{0}(\kappa(y))_{l^{n}}$. Note that $-(g-1) P_{c}$ modulo $z^{\times}$reduces to zero, for all $g \in G\left(c / c^{\prime}\right)$, as $K[c] / K\left[c^{\prime}\right]$ is totally ramified at $z^{\prime}$.

Denote by $\operatorname{Frob}(z)$ the Frobenius automorphism $x \mapsto x^{|\kappa(z)|}$ of the closed fiber $\mathcal{E}_{0} / \kappa(z)$ over $z$ of the Néron model of $E / F$. Theorem 4.8 .9 of [1] gives that for the prime $z^{\prime}$ of $K\left[c^{\prime}\right]$ above $z$ we have

$$
\operatorname{Frob}(z) y_{c} \equiv y_{c^{\prime}}\left(\bmod z^{\prime}\right)
$$

where $y_{c}=\left(0, I_{1}, c, \pi\right)$ as in (4.4.18).

We obtain from Proposition 4.3.1 that $y_{c} \bmod z^{\times}$is defined over the subfield $\kappa(y)$ of $\kappa\left(z^{\times}\right)$where $\kappa(y)$ is the quadratic extension field of the finite field $\kappa(z)$. Hence we have from (4.4.22) that

$$
\frac{\left|G\left(c / c^{\prime}\right)\right|}{l^{n}} y_{c}-\frac{a_{z}}{l^{n}} y_{c^{\prime}} \equiv \frac{\left|G\left(c / c^{\prime}\right)\right| \operatorname{Frob}(z)-a_{z}}{l^{n}} y_{c^{\prime}}\left(\bmod z^{\prime}\right) .
$$


The point $P_{c^{\prime}} \in E\left(K\left[c^{\prime}\right]\right)$ is given by (see Section 3.4.8)

$$
P_{c^{\prime}}=\sum_{s \in \mathcal{S}} s D_{c-z} y_{c^{\prime}}
$$

We then have from (4.4.21) and (4.4.23) that

$$
\begin{aligned}
Q & =\sum_{s \in \mathcal{S}} s D_{c-z}\left(\frac{\left|G\left(c / c^{\prime}\right)\right|}{l^{n}} y_{c}-\frac{a_{z}}{l^{n}} y_{c^{\prime}}\right) \\
& \equiv Q_{0} \equiv \frac{\left|G\left(c / c^{\prime}\right)\right| \operatorname{Frob}(z)-a_{z}}{l^{n}} P_{c^{\prime}}\left(\bmod z^{\prime}\right) .
\end{aligned}
$$

From Lemma 4.2.2 or [1, Lemma 7.14.11(ii)], we have that $P_{c^{\prime}}\left(\bmod l^{n}\right)$ belongs to the $-\nu\left(c^{\prime}\right)$-eigenspace for $\tau$ on $l^{n} E\left(K\left[c^{\prime}\right]\right)$, where $\nu\left(c^{\prime}\right)=(-1)^{r} \epsilon, r$ is the number of prime divisors in the support of $c^{\prime}$, and $\epsilon$ is the sign in the functional equation of the $L$-function of $E / F$ (as in Lemma 4.2.2). As $z$ is inert in $K / F$, it follows that the image of the reduction $P_{c^{\prime}}^{b}$ of $P_{c^{\prime}}$ modulo $z^{\prime}$ belongs to the $-\nu\left(c^{\prime}\right)$-eigenspace for $\tau$ on $l^{n} \mathcal{E}_{0}\left(\kappa\left(z^{\prime}\right)\right)$. Let

$$
h:{ }_{l^{n}} \mathcal{E}_{0}(\kappa(y)) \rightarrow \mathcal{E}_{0}(\kappa(y))_{l^{n}}, \quad x \mapsto\left(\frac{\left|G\left(c / c^{\prime}\right)\right| \operatorname{Frob}(z)-a_{z}}{l^{n}}\right) x
$$

be the isomorphism which commutes with $\tau$ of Proposition 4.4.1. As $Q_{0}$ belongs to the subgroup $\mathcal{E}_{0}(\kappa(y))$ as already noted and also as $P_{c^{\prime}}^{b}\left(\bmod l^{n}\right)$ is an element of $l^{n} \mathcal{E}_{0}(\kappa(y))$ by Proposition 4.3.2, we have by (4.4.24) that

$$
Q_{0}=h\left(P_{c^{\prime}}^{b}\left(\bmod l^{n}\right)\right)
$$

With the notation of (4.4.1) we then have

$$
Q_{0}=h \circ i\left(P_{c^{\prime}}\left(\bmod l^{n}\right)\right),
$$

where $i$ is the reduction isomorphism $l^{n} E\left(K_{y}\right) \rightarrow{ }_{l^{n}} \mathcal{E}_{0}(\kappa(y))$ and where $P_{c^{\prime}}\left(\bmod l^{n}\right)$ belongs to $l^{n} E\left(K_{y}\right)$ by Proposition 4.3.2. Furthermore, by (4.4.1) and as $\Phi=j \circ h \circ i$ we have that

$$
\Phi\left(P_{c^{\prime}}\left(\bmod l^{n}\right)\right)=j\left(Q_{0}\right)
$$

is the unique $l^{n}$-torsion point of $E\left(K_{y}\right)$ whose reduction at $y$ is $Q_{0}$.

Let $\bar{z}$ be any prime of $K^{\text {sep }}$ above $z^{\times}$. Restrict the cocycle

$$
\Gamma_{n}(c): \operatorname{Gal}\left(K^{\mathrm{sep}} / K\right) \rightarrow E\left(K^{\mathrm{sep}}\right)_{l^{n}}
$$

of (4.4.15), which represents $\gamma_{n}(c)$, to the decomposition group of $\bar{z}$. As $K[c] / K\left[c^{\prime}\right]$ is totally ramified at $z^{\prime}$ we have that the Kolyvagin element $D_{z}$ restricted to the residue field of $z^{\times}$satisfies

$$
D_{z}=-|\kappa(z)|(|\kappa(z)|+1) / 2 .
$$

Furthermore, $l^{n}$ divides $|\kappa(z)|+1$ and $l$ is different from 2 ; hence we have that (from (4.4.20)) the reduction $P_{c}^{b}$ of $P_{c}$ at $z^{\times}$satisfies $P_{c}^{b} \in l^{n} \mathcal{E}_{0}\left(\kappa\left(z^{\times}\right)\right)$and hence as $E$ has good reduction at $z^{\times}$we have that

$$
P_{c} \in l^{n} E\left(K[c]_{z^{\times}}\right) .
$$


We then have that the cocycle $\Gamma_{n}(c)$ of (4.4.15) satisfies

$$
\Gamma_{n}(c)(\rho)=0 \quad \text { for all } \rho \in \operatorname{Gal}\left(K[c]_{z^{\times}}^{\mathrm{sep}} / K[c]_{z^{\times}}\right),
$$

and this cocycle, restricted to the decomposition group of $\bar{z}$, factors through the $\operatorname{subgroup} \operatorname{Gal}\left(K[c]_{z^{\times}} / K_{y}\right)$. Furthermore, since $\sigma_{z}$, which is the selected generator of $G\left(c / c^{\prime}\right)$, is in the inertia group of $y$ we have that

$$
\sigma_{z} \frac{P_{c}}{l^{n}}-\frac{P_{c}}{l^{n}}
$$

reduces to zero modulo $z^{\times}$. Hence,

$$
\Gamma_{n}(c)\left(\sigma_{z}\right)=-\frac{\left(\sigma_{z}-1\right) P_{c}}{l^{n}}+\sigma_{z} \frac{P_{c}}{l^{n}}-\frac{P_{c}}{l^{n}}
$$

is the unique $l^{n}$-torsion point congruent to $Q\left(\bmod z^{\times}\right)$where $Q$ is given by (4.4.17); that is to say,

$$
\Gamma_{n}(c)\left(\sigma_{z}\right) \equiv-\frac{\left(\sigma_{z}-1\right) P_{c}}{l^{n}}\left(\bmod z^{\times}\right) .
$$

But from (4.4.25), we then obtain

$$
\Gamma_{n}(c)\left(\sigma_{z}\right)=\Phi\left(P_{c^{\prime}}\left(\bmod l^{n}\right)\right) .
$$

Then (4.4.27) shows, as $\Gamma_{n}(c)\left(\sigma_{z}\right)$ is the unique $l^{n}$-torsion point with reduction at $y$ coinciding with the reduction of $Q=-\left[\left(\sigma_{z}-1\right) P_{c}\right] / l^{n}$ at $y$, that we have an equality of cohomology classes in $H^{1}\left(K_{y}, E_{l^{n}}\right)$

$$
\gamma_{n}(c)_{y}=\chi_{z}\left(P_{c-z}\left(\bmod l^{n}\right)\right),
$$

where $P_{c-z}\left(\bmod l^{n}\right)$ is an element of $l^{n} E\left(K_{y}\right)$. This proves property $(\mathrm{d})$ and completes the proof of Proposition 4.4.2.

\section{REMARKS 4.4.3}

(a) Proposition 4.4.2 and its consequence stated in Proposition 4.5.1(d) below are extensions of [1, Lemma 7.14.14(ii)]. It would be of interest to eliminate the hypothesis that the prime number $l$ be coprime to the order of the Picard group $\operatorname{Pic}(A)$ from Propositions 4.4.2 and 4.5.1(d).

(b) The homomorphism $\chi_{z}$ interchanges the $\tau$-eigenspaces such that for $\delta= \pm 1$ we have

$$
\chi_{z}\left(\left(l^{n} E\left(K_{y}\right)\right)^{\delta}\right) \subseteq H^{1}\left(K_{y}, E_{l^{n}}\right)^{-\delta} .
$$

This property, which is not required for this paper, follows from the group $\operatorname{Gal}(K[c] / F)$ being generalized dihedral.

\subsection{Localizations of the classes $\gamma_{n}(c)$ and $\delta_{n}(c)$}

The notation and hypotheses of Section 4.1.1 hold in this section. Let $\amalg(E / K)$ be the Tate-Shafarevich group of the elliptic curve $E \times_{F} K$ over $K$. 


\section{PROPOSITION 4.5.1}

Let $c \in \Lambda(n)$, let $z$ be a prime divisor in $\Lambda^{1}(n)$, and let $y$ be the place of $K$ over the place $z$ of $F$.

(a) Let $v$ be a place of $K$ coprime to $c$. Then we have $\gamma_{n}(c)_{v} \in \partial_{n}\left(E\left(K_{v}\right)\right)$; that is to say, we have $\delta_{n}(c)_{v}=0$.

(b) If $c$ is coprime to $z$, then we have $\gamma_{n}(c)_{y}=\partial_{n}\left(\left(P_{c}\left(\bmod l^{n}\right)\right)_{y}\right)$.

(c) If $l^{n} \mid P_{c}$, then we have $\delta_{n}(c)=0$. If $l^{n} \mid P_{c-w}$ for all prime divisors $w$ in the support of $c$, then we have $\delta_{n}(c) \in \amalg(E / K)_{l^{\infty}}$.

(d) Assume that the prime number $l \in \mathcal{P}$ is coprime to the order of $\operatorname{Pic}(A)$, the Picard group of the ring A. If $z \in \operatorname{Supp}(c)$, then we have

$$
\begin{aligned}
\operatorname{ord} \delta_{n}(c)_{y} & =\operatorname{ord} \gamma_{n}(c)_{y}=\operatorname{ord} \gamma_{n}(c-z)_{y} \\
& =\operatorname{ord}\left(\left(P_{c-z}\left(\bmod l^{n}\right)\right)_{y}\right),
\end{aligned}
$$

where $\left(P_{c-z}\left(\bmod l^{n}\right)\right)_{y}$ is an element of $l^{n} E\left(K_{y}\right)$.

Proof

(a) The field $K[c]$ is a subfield of $K_{\infty}$ as $\infty$ is split completely in $K[c] / K$ (see [1, Chapter 2, (2.3.13)]). We have that the localization $\delta_{n}(c)_{\infty}$ at $\infty$ of $\delta_{n}(c)$ satisfies $\delta_{n}(c)_{\infty} \in H^{1}\left(K_{\infty}, E\right)_{l^{n}}$ and the localization $\left(P_{c}\left(\bmod l^{n}\right)\right)_{\infty}$ at $\infty$ of $P_{c}\left(\bmod l^{n}\right)$ satisfies $\left(P_{c}\left(\bmod l^{n}\right)\right)_{\infty} \in l^{n} E\left(K_{\infty}\right)$. It then follows from the diagram (3.4.2) that $\delta_{n}(c)_{\infty}=0$.

Suppose now that $v$ is a place of $K$ such that $v \neq \infty$ and $v \notin \operatorname{Supp}(c)$. We have by construction that

$$
\delta_{n}(c) \in H^{1}(K[c] / K, E(K[c]))_{l^{n}} .
$$

The field extension $K[c] / K$ is unramified at $v$ (see $[1,(2.3 .13)]$ ). Hence we have that the localization $\delta_{n}(c)_{v}$ at $v$ satisfies

$$
\delta_{n}(c)_{v} \in H^{1}\left(K_{v}^{n r} / K_{v}, E\left(K_{v}^{n r}\right)\right)_{l^{n}} \subseteq H^{1}\left(K_{v}, E\left(K_{v}^{\mathrm{sep}}\right)\right)_{l^{n}},
$$

where $K_{v}^{n r}$ is the maximal unramified separable extension of the local field $K_{v}$. But $H^{1}\left(K_{v}^{n r} / K_{v}, E\left(K_{v}^{n r}\right)\right)_{l^{n}}=0$ by Definition 3.1.2(d) of the set of prime numbers $\mathcal{P}$ to which $l$ belongs. Hence, we have that $\delta_{n}(c)_{v}=0$; this last vanishing is equivalent to $\gamma_{n}(c)_{v} \in \partial_{n}\left(E\left(K_{v}\right)\right)$, as required.

(b) By Lemma 4.2.1, the cohomology class $\gamma_{n}(c)$ is represented by the cocycle

$$
\sigma \mapsto-\frac{(\sigma-1) P_{c}}{l^{n}}+\sigma \frac{P_{c}}{l^{n}}-\frac{P_{c}}{l^{n}}, \quad \operatorname{Gal}\left(K^{\mathrm{sep}} / K\right) \rightarrow E\left(K^{\mathrm{sep}}\right)_{l^{n}},
$$

where $\left[(\sigma-1) P_{c}\right] / l^{n}$ is the unique $l^{n}$-division point of $(\sigma-1) P_{c}$ in $E(K[c])$. Furthermore, by the same Lemma 4.2.1, the cohomology class $\delta_{n}(c) \in$ $H^{1}\left(K, E\left(K^{\mathrm{sep}}\right)\right)_{l^{n}}$ is represented by the cocycle

$$
\sigma \mapsto-\frac{(\sigma-1) P_{c}}{l^{n}}, \quad \operatorname{Gal}\left(K^{\mathrm{sep}} / K\right) \rightarrow E\left(K^{\mathrm{sep}}\right) .
$$

By part (a) as $z \notin \operatorname{Supp}(c)$, we have that $\delta_{n}(c)_{y}=0$; hence, a cocycle representing $\delta_{n}(c)$ when localized at $v$ is cohomologous to zero. Therefore, there is an element 
$a \in E\left(K_{y}^{\mathrm{sep}}\right)$ such that

$$
-\frac{(\sigma-1) P_{c}}{l^{n}}=\sigma a-a, \quad \text { for all } \sigma \in \operatorname{Gal}\left(K_{y}^{\text {sep }} / K_{y}\right) .
$$

Hence, we have

$$
-(\sigma-1)\left(P_{c}+l^{n} a\right)=0, \quad \text { for all } \sigma \in \operatorname{Gal}\left(K_{y}^{\text {sep }} / K_{y}\right) .
$$

This implies that

$$
P_{c}+l^{n} a \in E\left(K_{y}\right) .
$$

By Proposition 4.3.1, the localization at $y$ of the point $P_{c}$ lies in $E\left(K_{y}\right)$ where $y$ is the place of $K$ over $z$. Furthermore, by Proposition 4.3.2 the point $\left(P_{c}\left(\bmod l^{n}\right)\right)_{y}$ belongs to $l^{n} E\left(K_{y}\right)$ and its image in this group is uniquely determined by $P_{c}$.

It follows from this and the above cocycle formulae for $\gamma_{n}(c)$ and $\delta_{n}(c)$ that the cohomology class $\gamma_{n}(c)_{y}$ is represented by the cocyle

$$
\sigma \mapsto \sigma \frac{P_{c}}{l^{n}}-\frac{P_{c}}{l^{n}}, \quad \operatorname{Gal}\left(K_{y}^{\mathrm{sep}} / K_{y}\right) \rightarrow E\left(K_{y}^{\mathrm{sep}}\right)_{l^{n}} .
$$

That is to say, we have $\gamma_{n}(c)_{y}=\partial_{n}\left(\left(P_{c}\left(\bmod l^{n}\right)\right)_{y}\right)$.

(c) From Lemma 4.2.1(b), the cohomology class $\delta_{n}(c)$ is represented by the cocycle

$$
\sigma \mapsto-\frac{(\sigma-1) P_{c}}{l^{n}}, \quad \operatorname{Gal}\left(K^{\mathrm{sep}} / K\right) \rightarrow E\left(K^{\mathrm{sep}}\right) .
$$

We then evidently have that if $l^{n} \mid P_{c}$, that is to say, $P_{c} \in l^{n} E(K[c])$, then $\delta_{n}(c)=0$.

Suppose that $l^{n} \mid P_{c-z}$ for all prime divisors $z$ in the support of $c$. Then for any prime divisor $y$ of $K$ lying over the prime divisor $z$ dividing $c$ we have $\delta_{n}(c)_{y}=$ 0 by Proposition 4.4.2(d). It then follows from Proposition 4.5.1(a) that $\delta_{n}(c)_{v}=$ 0 for all places $v$ of $K$ and hence that $\delta_{n}(c)$ belongs to the Tate-Shafarevich group $\amalg(E / K)_{l \infty}$.

(d) From Proposition 4.4.2(d), we have

$$
\gamma_{n}(c)_{y}=\chi_{z}\left(\left(P_{c-z}\left(\bmod l^{n}\right)\right)_{y}\right)
$$

where $\left(P_{c-z}\left(\bmod l^{n}\right)\right)_{y}$ is an element of $l^{n} E\left(K_{y}\right)$ by Proposition 4.3.2. As the homomorphism $\chi_{z}$ is injective (Proposition 4.4.2(b)), we obtain

$$
\operatorname{ord}\left(\gamma_{n}(c)_{y}\right)=\operatorname{ord}\left(\left(P_{c-z}\left(\bmod l^{n}\right)\right)_{y}\right)
$$

where again $\left(P_{c-z}\left(\bmod l^{n}\right)\right)_{y}$ denotes an element of $l^{n} E\left(K_{y}\right)$.

From Proposition 4.5.1(b), we have

$$
\gamma_{n}(c-z)_{y}=\partial_{n}\left(\left(P_{c-z}\left(\bmod l^{n}\right)\right)_{y}\right),
$$

where $z$ is coprime to the support of $c-z$. Hence, we obtain

$$
\operatorname{ord}\left(\left(P_{c-z}\left(\bmod l^{n}\right)\right)_{y}\right)=\operatorname{ord}\left(\gamma_{n}(c-z)_{y}\right) .
$$


From Proposition 4.4.2(c), we have that the composition of $\chi_{z}$ with the homomorphism, obtained from the inclusion of group schemes $E_{l^{n}} \subset E$,

$$
\theta: H^{1}\left(K_{y}, E_{l^{n}}\right) \rightarrow H^{1}\left(K_{y}, E\right)_{l^{n}}
$$

is an isomorphism

$$
l^{n} E\left(K_{y}\right) \cong H^{1}\left(K_{y}, E\right)_{l^{n}}
$$

Hence, the image in $H^{1}\left(K_{y}, E\right)_{l^{n}}$ under $\theta$ of $\chi_{z}\left(P_{c-z}\left(\bmod l^{n}\right)\right)$ is the cohomology class $\delta_{n}(c)_{y}$ because this is the image under $\theta$ of $\gamma_{n}(c)_{y} \in H^{1}\left(K_{y}, E_{l^{n}}\right)$. Furthermore, because $\theta \circ \chi_{z}$ is an isomorphism, $\operatorname{ord}\left(\delta_{n}(c)_{y}\right)$ is the same as $\operatorname{ord}\left(\left(P_{c-z}\left(\bmod l^{n}\right)\right)_{y}\right)$, which completes the proof of the proposition.

\subsection{The Cassels pairing with a class $\delta_{n}(c)$}

The notation and hypotheses of Section 4.1.1 hold in this section. The torsion abelian group $\mathbb{Z} / n \mathbb{Z}$ for $n \neq 0$ is considered to be a subgroup of $\mathbb{Q} / \mathbb{Z}$ via the map $1 \mapsto 1 / n$. Let

$\amalg(E / K)$ be the Tate-Shafarevich group of the elliptic curve $E \times_{F} K$ over $K$;

$\langle\cdot, \cdot\rangle_{\text {Cassels }}$ be the Cassels pairing on $\amalg(E / K)$ (see Section 2.3);

$[\cdot, \cdot]_{w}: H^{1}\left(K_{w}, E\right)_{n} \times{ }_{n} E\left(K_{w}\right) \rightarrow \mathbb{Z} / n \mathbb{Z}$ be the local Tate pairing for any place $w \in \Sigma_{K}$ of $K$ and any integer $n$ coprime to the characteristic of $F$.

\section{PROPOSITION 4.6.1}

Let $m$ and $n$ be integers greater than or equal to 1 , and let $c \in \Lambda(m+n)$. Suppose that $\delta_{m}(c)$ belongs to the Tate-Shafarevich group $\amalg(E / K)_{l \infty}$. Suppose that the element $s \in \amalg(E / K)_{l_{\infty}}$ has order at most $l^{n}$. Lift the element $s \in \amalg(E / K)_{l^{\infty}}$ to an element $S \in H^{1}\left(K, E_{l^{n}}\right)$, and select points $x(w) \in E\left(K_{w}\right)$ such that $S_{w}=$ $\partial_{l^{n}}(x(w))$ for all $w \in \Sigma_{K}$. Then we have

$$
\left\langle\delta_{m}(c), s\right\rangle_{\text {Cassels }}=\sum_{y \in \Sigma_{K}} \sum_{\text {divides } \operatorname{Supp}(c)}\left[\delta_{m+n}(c)_{y}, x(y)\right]_{y},
$$

where the sum runs over the places of $K$ which divide an element of $\operatorname{Supp}(c)$.

\section{Proof}

The construction of the Cassels pairing is given in Section 2.3. As in the statement of the proposition, we may lift the element $s \in \amalg(E / K)_{l^{\infty}}$ of order $l^{n}$ to an element $S \in H^{1}\left(K, E_{l^{n}}\right)$. The points $x(w) \in E\left(K_{w}\right)$ are then selected such that $S_{w}=\partial_{l^{n}}(x(w))$ for all $w \in \Sigma_{K}$.

By Lemma 4.2.1(c) we have

$$
l^{n} \delta_{m+n}(c)=\delta_{m}(c) .
$$

From the formula (2.3.1) for the Cassels pairing, we then obtain 


$$
\left\langle\delta_{m}(c), s\right\rangle_{\mathrm{Cassels}}=\sum_{w \in \Sigma_{K}}\left[\delta_{m+n}(c)_{w}, x(w)\right]_{w},
$$

where the sum runs over all places $w$ of $K$.

By Proposition 4.5.1(a), we have that $\delta_{m+n}(c)_{w}=0$ for all places $w \in \Sigma_{K}$ which do not divide an element of $\operatorname{Supp}(c)$. Hence, there is no contribution to the Cassels pairing in the sum (4.6.2) when $w$ does not divide an element of $\operatorname{Supp}(c)$. Hence, we obtain the formula (4.6.1).

\section{PROPOSITION 4.6.2}

Let $m$ and $n$ be integers greater than or equal to 1 , and let $c \in \Lambda(m+n), d \in \Lambda(n)$. Suppose that $\delta_{m}(c)$ and $\delta_{n}(d)$ belong to the Tate-Shafarevich group $\amalg(E / K)_{l^{\infty}}$. Assume that the prime number $l \in \mathcal{P}$ is coprime to the order of $\operatorname{Pic}(A)$. Then we have

$$
\begin{aligned}
& \left\langle\delta_{m}(c), \delta_{n}(d)\right\rangle_{\text {Cassels }} \\
& =\sum_{y \in \Sigma_{K}} \sum_{\text {divides } \operatorname{Supp}(c) \backslash \operatorname{Supp}(d)}\left[\delta_{m+n}(c)_{y},\left(P_{d}\left(\bmod l^{n}\right)\right)_{y}\right]_{y},
\end{aligned}
$$

where the sum runs over the places of $K$ which divide an element of $\operatorname{Supp}(c) \backslash$ $\operatorname{Supp}(d)$.

Proof

The element $\gamma_{n}(d) \in H^{1}\left(K, E_{l^{n}}\right)$ is a lifting of $\delta_{n}(d) \in \amalg(E / K)$, which has order at most $l^{n}$. Suppose that $z \in \operatorname{Supp}(c)$, and suppose that $y$ is the unique place of $K$ lying over the place $z$ of $F$. If $z$ also satisfies $z \in \operatorname{Supp}(d)$, then by Proposition 4.5.1(d), we have

$$
\gamma_{n}(d)_{y}=0
$$

because $\delta_{n}(d)_{y}=0$ as we have $\delta_{n}(d) \in \amalg(E / K)$. If on the other hand $z$ satisfies $z \notin \operatorname{Supp}(d)$, then by Proposition 4.5.1(b), we have

$$
\gamma_{n}(d)_{y}=\partial_{l^{n}}\left(\left(P_{d}\left(\bmod l^{n}\right)\right)_{y}\right)
$$

where $\left(P_{d}\left(\bmod l^{n}\right)\right)_{y}$ denotes the localization at $y$ of $P_{d}\left(\bmod l^{n}\right)$. The formula (4.6.3) to be proved now follows from the formula (4.6.1) of Proposition 4.6.1.

\section{Part 5. Construction of cohomology classes and proofs of the main theorems}

\section{1. $M_{r}$ is finite for some $r$}

5.1.1.

Throughout Part 5, the notation of Section 4.1.1 remains valid, and the elliptic curve $E / F$ and quadratic field extension $K / F$ satisfy hypotheses (a), (b), and (c) of Section 4.1.1.

In this section, we further let

$N^{+}, N^{-}$be the eigenspaces under the action of the involution $\tau$ whenever $N$ is a $\mathbb{Z}[\operatorname{Gal}(K / F)]$-module on which multiplication by 2 is an isomorphism; 
$\epsilon= \pm 1$ be the sign of the functional equation of the $L$-function $L(E / F, s)$ of $E / F$;

$\nu(r)=(-1)^{r} \epsilon$ for any natural number $r \in \mathbb{N}$;

$\nu(c)=(-1)^{r} \epsilon$ for any divisor $c$ of $F$ with exactly $r$ distinct prime divisors in its support;

$P_{c} \in E(K[c])$ be the Drinfeld-Heegner points of Section 3.4.8 for all $c \in \Lambda(1)$, where $P_{0} \in E(K)$;

$M_{r}$, for all $r \in \mathbb{N}$, be the quantities in $\mathbb{N} \cup\{+\infty\}$ given in Definition 4.1.1;

$\amalg(E / K)$ be the Tate-Shafarevich group of the elliptic curve $E \times_{F} K$ over $K$ (see Section 2.2);

$\operatorname{Sel}_{n}(E / K)$ be the $n$-Selmer group of $E \times{ }_{F} K$ over $K$ for any integer $n$ coprime to the characteristic of $F$ (see Section 2.2);

$\operatorname{Sel}_{a} \infty(E / K)=\underset{n}{\lim _{\longrightarrow}} \operatorname{Sel}_{a^{n}}(E / K)$ for any number $a$ coprime to the characteristic of $F$ (see also Section 3.1.2).

In Section 5.1 some consequences are presented of the hypothesis that $M_{r}$ be finite for some $r$.

\section{LEMMA 5.1.1}

The Drinfeld-Heegner $P_{0}$ has infinite order in $E(K)$ if and only if $M_{0}$ is finite. If $P_{0}$ has infinite order, then we have

$$
\begin{aligned}
l^{M_{0}} & =\left|\left(E(K) / \mathbb{Z} P_{0}\right)_{l^{\infty}}\right|, \\
\text { ord } \gamma_{M_{0}+m}(0) & =l^{m}, \quad \text { for all } m \geq 0, \\
\gamma_{M_{0}+m}(0) & \in \operatorname{Sel}_{l \infty}(E / K)^{-\epsilon} \quad \text { for all } m \geq 0 .
\end{aligned}
$$

Proof

From Definition 4.1.1, we have

$$
M_{0}=\operatorname{ord}_{l}\left(P_{0}\right)=\max \left\{m \mid P_{0} \in l^{m} E(K[0])\right\} .
$$

The group $E(K[0]$ ) has no $l$-torsion (as $l \in \mathcal{P}$; see Proposition 1.10 .1 and Definition 3.1.2(f)) and is a finitely generated group by the Mordell-Weil theorem. Hence, $P_{0}$ has infinite order in $E(K)$ if and only if $M_{0}$ is finite.

We have $\gamma_{M_{0}+m}(0) \in \operatorname{Sel}_{l \infty}(E / K)^{-\epsilon}$, for all $m \geq 1$, by Lemma 4.2 .2 and because $\gamma_{M_{0}+m}(0)=\partial_{l^{M_{0}+m}}\left(P_{0}\right)$.

Assume now that $P_{0}$ has infinite order. By [1, Theorem 7.6.5], the point $P_{0}$ generates a subgroup of $E(K)$ of finite index. (Note that, in the notation of [1, Theorem 7.6.5], we have $P_{0}=x_{0}$.)

By definition we have that

$$
\left|\left(E(K) / \mathbb{Z} P_{0}\right)_{l^{\infty}}\right|=\max \left\{l^{m} \mid P_{0} \in l^{m} E(K)\right\},
$$

where the group $E\left(K[0]\right.$ ) has no $l^{\infty}$-torsion (as $l \in \mathcal{P}$; see Proposition 1.10 .1 and Definition 3.1.2(f)). 
We have the Hochschild-Serre spectral sequence

$$
H^{i}\left(\operatorname{Gal}(K[0] / K), H^{j}\left(K[0], E_{l^{n}}\right)\right) \Rightarrow H^{i+j}\left(K, E_{l^{n}}\right) .
$$

The short exact sequence of low-degree terms of this spectral sequence is in part

$$
\begin{aligned}
0 & \rightarrow H^{1}\left(\operatorname{Gal}(K[0] / K), E_{l^{n}}(K[0])\right) \rightarrow H^{1}\left(K, E_{l^{n}}\right) \rightarrow H^{1}\left(K[0], E_{l^{n}}\right)^{\operatorname{Gal}(K[0] / K)} \\
& \rightarrow H^{2}\left(\operatorname{Gal}(K[0] / K), E_{l^{n}}(K[0])\right) .
\end{aligned}
$$

The two extreme terms $H^{1}\left(\operatorname{Gal}(K[0] / K), E_{l^{n}}(K[0])\right)$ and $H^{2}(\operatorname{Gal}(K[0] / K)$, $\left.E_{l^{n}}(K[0])\right)$ are both zero because $E_{l^{n}}(K[0])$ is zero as already noted. Hence, this short exact sequence provides the isomorphism

$$
H^{1}\left(K, E_{l^{n}}\right) \cong H^{1}\left(K[0], E_{l^{n}}\right)^{\operatorname{Gal}(K[0] / K)},
$$

which is induced from the injection $E(K) \rightarrow E(K[0])$. The short exact sequence of sheaves for the étale topology on $\operatorname{Spec} K$

$$
0 \longrightarrow E_{l^{n}} \longrightarrow E \stackrel{l^{n}}{\longrightarrow} E \longrightarrow 0
$$

then provides the commutative diagram of cohomology groups

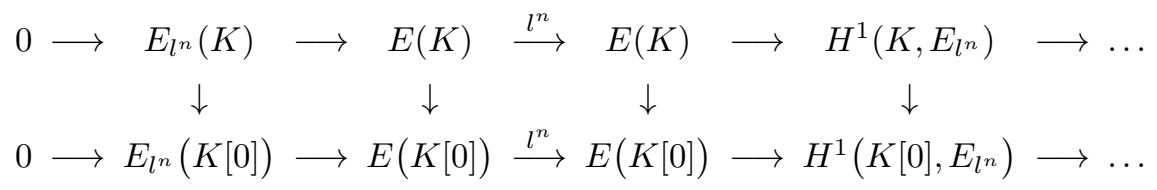

The isomorphism $H^{1}\left(K, E_{l^{n}}\right) \cong H^{1}\left(K[0], E_{l^{n}}\right)^{\mathrm{Gal}(K[0] / K)}$ of (5.1.1) together with this commutative diagram then shows that the homomorphism

$$
E(K) / l^{n} E(K) \rightarrow E(K[0]) / l^{n} E(K[0])
$$

is injective. Hence, these two numbers $l^{M_{0}}$ and $\left|\left(E(K) / \mathbb{Z} P_{0}\right)_{l}\right|$ are equal. We obtain that the order of $\gamma_{M_{0}+m}(0)$ is equal to $l^{m}$ from Proposition 3.4.1(a).

\section{LEMMA 5.1.2}

Assume that the prime number $l \in \mathcal{P}$ is coprime to the order of $\operatorname{Pic}(A)$. Suppose that $M_{r}$ is finite for some integer $r \geq 0$. Then $M_{s}$ is finite for all $s \geq r$ and

$$
M_{r}, M_{r+1}, M_{r+2}, \ldots
$$

is a decreasing sequence of nonnegative integers.

Proof

Suppose that $M_{s}$ is finite for some $s \geq 0$. Then there is a divisor

$$
c \in \Lambda^{s}\left(M_{s}+1\right)
$$

which satisfies $l^{M_{s}} \| P_{c}$ and $M_{s}<\alpha(c)$; hence, the cohomology class $\gamma_{M_{s}+1}(c)$ is nonzero (by Proposition 3.4.1(a)). From Proposition 3.3.4, where we take $n=$ $M_{s}+1$, we obtain a prime divisor

$$
z \in \Lambda^{1}\left(M_{s}+1\right)
$$


coprime to $c$ such that the localization $\gamma_{M_{s}+1}(c)_{y}$ is nonzero where $y$ is the unique prime of $K$ lying over $z$ (see Remark 3.2.2(a)). Then by Proposition 4.5.1(b), we have that $P_{c} \notin l^{M_{s}+1} E\left(K_{y}\right)$; that is to say,

$$
\operatorname{ord}\left(\left(P_{c}\left(\bmod l^{M_{s}+1}\right)\right)_{y}\right)>1 .
$$

It follows from Proposition 4.5.1(d) that ord $\gamma_{n}(c+z)_{y}>1$, where $n=M_{s}+1$, and in particular we have $\gamma_{n}(c+z) \neq 0$. Therefore, by Proposition 3.4.1(a) or the definition of $\gamma_{n}(c+z)$, we have that $P_{c+z} \notin l^{M_{s}+1} E(K[c+z])$. It follows that

$$
c+z \in \Lambda^{s+1}\left(M_{s}+1\right)
$$

and $M_{s+1}$ is finite, and we have that $M_{s+1} \leq M_{s}$. As $M_{r}$ is finite by hypothesis, we then have that $M_{s}$ is finite and $M_{s} \geq M_{s+1}$ for all $s \geq r$ by induction.

LEMMA 5.1.3

Suppose that $M_{r-1}$ is finite where $r \geq 1$. Assume that the prime number $l \in \mathcal{P}$ is coprime to the order of $\operatorname{Pic}(A)$. Let $c \in \Lambda^{r}\left(M_{r-1}\right)$, and put $\nu(c)=(-1)^{r} \epsilon$. Then we have that
(a) $\delta_{M_{r-1}}(c) \in \amalg(E / K)_{l^{\infty}}^{-\nu(c)}$;
(b) $\gamma_{M_{r-1}}(c) \in \operatorname{Sel}_{l^{\infty}}(E / K)^{-\nu(c)}$; and
(c) the order of $\delta_{M_{r-1}}(c)$ is at most $l^{M_{r-1}-M_{r}}$.

Proof

By Lemma 5.1.2, we have that $M_{r}$ is finite and $M_{r-1} \geq M_{r}$. The set $\Lambda^{r}\left(M_{r-1}\right)$ is nonempty by Corollary 3.3.5; therefore, there is an element $c \in \Lambda^{r}\left(M_{r-1}\right)$. It follows from the definition of the $M_{i}$ 's that $l^{M_{r}} \mid P_{c}$ and $l^{M_{r-1}} \mid P_{c-z}$ for all prime divisors $z$ in the support of $c$. From Proposition 4.5.1(c) and Lemma 4.2.2 we obtain

$$
\delta_{M_{r-1}}(c) \in \amalg(E / K)^{-\nu(c)} .
$$

Hence, $\gamma_{M_{r-1}}(c)$ belongs to the Selmer group $\operatorname{Sel}_{l \infty}(E / K)^{-\nu(c)}$ (see Lemma 4.2.2). The order of the element $\delta_{M_{r-1}}(c)$ is at most $l^{M_{r-1}-M_{r}}$ by Proposition 3.4.1(b).

\section{LEMMA 5.1.4}

Let $z \in \Lambda^{1}(n)$ be a prime divisor. Let $y$ be the unique prime of $K$ lying above $z$.

(a) The Tate pairing on $H^{1}\left(K_{y}, E_{l^{n}}\right)$ of Theorem 2.1.1 induces a nondegenerate pairing

$$
\left({ }_{l^{n}} E\left(K_{y}\right)\right)^{\delta} \times H^{1}\left(K_{y}, E\right)_{l^{n}}^{\delta} \longrightarrow \mathbb{Z} / l^{n} \mathbb{Z},
$$

where $\delta= \pm 1$, of eigenspaces under the action of $\tau$ which are finite cyclic groups of order $l^{n}$.

(b) The image of the homomorphism $\chi_{z}: l^{n} E\left(K_{y}\right) \rightarrow H^{1}\left(K_{y}, E_{l^{n}}\right)$ is an isotropic subgroup for the alternating Tate pairing on $H^{1}\left(K_{y}, E_{l^{n}}\right)$ and $\operatorname{Im}\left(\chi_{z}\right)^{\delta} \cong$ $\mathbb{Z} / l^{n} \mathbb{Z}$ for $\delta= \pm 1$. 
Proof

(a) We have isomorphisms of $\operatorname{Gal}(K / F)$-modules

$$
l^{n} E\left(K_{y}\right) \cong E\left(K_{y}\right)_{l^{n}}, \quad H^{1}\left(K_{y}, E\right)_{l^{n}} \cong \operatorname{Hom}\left(\mu_{l^{n}}\left(K_{l^{n}}\right), E\left(K_{y}\right)\right) .
$$

The first isomorphism here follows from Proposition 4.4.1. As $|\kappa(z)|+1 \equiv$ $0\left(\bmod l^{n}\right)$ and $\kappa(y)^{*}$ has $|\kappa(z)|^{2}-1$ elements we have that $\mu_{l^{n}}\left(K_{y}\right)$ has $l^{n}$ elements and is contained in the -1 eigenspace under $\tau$ of $\kappa(y)^{*}$. Hence, we have for $\delta= \pm 1$

$$
{ }_{l^{n}} E\left(K_{y}\right)^{\delta} \cong H^{1}\left(K_{y}, E\right)_{l^{n}}^{-\delta}
$$

and these groups are cyclic of order $l^{n}$ by Lemma 3.2.3(b).

(b) The map $\chi_{z}$ is defined in Proposition 4.4.2. We have by Proposition 4.4.2(c) that the composite of $\chi_{z}$ with the surjective homomorphism $H^{1}\left(K_{y}, E_{l^{n}}\right) \rightarrow$ $H^{1}\left(K_{y}, E\right)_{l^{n}}$ is an isomorphism. This implies that we have for $\delta= \pm 1$, where $\operatorname{Im}\left(\chi_{z}\right)$ denotes the image of $\chi_{z}$ in $H^{1}\left(K_{y}, E_{l^{n}}\right)$,

$$
\operatorname{Im}\left(\chi_{z}\right)^{\delta} \cong H^{1}\left(K_{y}, E\right)_{l^{n}}^{\delta}
$$

Hence, part (a) on Tate duality implies that for $\delta= \pm 1$

$$
\operatorname{Im}\left(\chi_{z}\right)^{\delta} \cong \mathbb{Z} / l^{n} \mathbb{Z}
$$

This evidently shows that $\operatorname{Im}\left(\chi_{z}\right)^{\delta}$ is an isotropic subgroup of $H^{1}\left(K_{y}, E_{l^{n}}\right)$ for the antisymmetric Tate pairing. Since the cup product on $H^{1}\left(K_{y}, E_{l^{n}}\right)$ is $\operatorname{Gal}(K / F)$ equivariant it follows that $\operatorname{Im}\left(\chi_{z}\right) \cong \operatorname{Im}\left(\chi_{z}\right)^{+1} \oplus \operatorname{Im}\left(\chi_{z}\right)^{-1}$ is an isotropic subgroup of $H^{1}\left(K_{y}, E_{l^{n}}\right)$.

\section{LEMMA 5.1.5}

Let $z \in \Lambda^{1}(n)$ be a prime divisor where $n \geq 1$. Let $y$ be the unique prime of $K$ lying above $z$. Let $S$ be a finite set of prime divisors of $\Lambda^{1}(n)$ not containing $z$. Let $\delta= \pm 1$. Then there is a nonzero element $h \in H^{1}\left(K, E_{l^{n}}\right)^{\delta}$ in the $\delta$-eigenspace satisfying these two conditions:

(a) $h_{x} \in \partial_{l^{n}}\left(E\left(K_{x}\right)\right)$ for any place $x$ of $K$ not lying over a place of $S \cup\{z\}$;

(b) $h_{x} \in \operatorname{Im}\left(\chi_{w}\right)$ for all $w \in S$ where $x$ is the unique place of $K$ over $w$ and where $\chi_{w}: l^{n} E\left(K_{x}\right) \rightarrow H^{1}\left(K_{x}, E_{l^{n}}\right)$ is the homomorphism of Proposition 4.4.2.

\section{Proof}

The places of $S$ remain inert in the field extension $K / F$; for any $u \in S$ denote by $u^{\sharp}$ the corresponding place of $K$ over $u$. For any place $v \in \Sigma_{K}$ of $K$ we have the exact sequence

$$
0 \longrightarrow l^{n} E\left(K_{v}\right) \stackrel{\partial_{l^{n}}}{\longrightarrow} H^{1}\left(K_{v}, E_{l^{n}}\right) \longrightarrow H^{1}\left(K_{v}, E\right)_{l^{n}} \longrightarrow 0
$$

where the extremities of this sequence are in duality by Theorem 2.1.2.

Let $\delta= \pm 1$. The elliptic curve $E / F$ has good reduction at all places of $S \cup\{z\}$ (see Remarks 3.2.2). Let $U$ be the finite subset of $\Sigma_{F}$ of places of $F$ given by

$$
U=S \cup\{z\} \cup\left\{\text { bad reduction places of } E / F \text { in } \Sigma_{F}\right\} \text {. }
$$


Let $U_{K}$ be the finite set of all places of $K$ over the places of $U$. For all $v \in U_{K}$ dividing an element $u$ of $U \backslash\{z\}$ put

$$
\begin{aligned}
& H_{v}=\operatorname{Im}\left(\chi_{u}\right)^{\delta} \quad \text { if } u \in S, \\
& H_{v}=\partial_{l^{n}}\left(E\left(K_{v}\right)\right)^{\delta} \quad \text { if } u \in U \backslash(S \cup\{z\}) .
\end{aligned}
$$

Then we have

$$
\left|H_{v}\right|^{2}=\left|H^{1}\left(K_{v}, E_{l^{n}}\right)^{\delta}\right| \text { for all } v \text { dividing a place } u \in U \backslash\{z\} .
$$

For the case where $u \in S$ this follows from Lemma 5.1.4(b). For the case where $u \in U \backslash(S \cup\{z\})$, that is to say, a place of bad reduction, this follows from the exact sequence (5.1.4) and that the extremities of this sequence are in duality.

From Tate local and global duality, there is a self-dual exact sequence (see [10, Chapter I, Theorem 4.10, p. 70])

$$
H^{1}\left(K_{U} / K, E_{l^{n}}\right) \rightarrow \bigoplus_{v \in U_{K}} H^{1}\left(K_{v}, E_{l^{n}}\right) \rightarrow H^{1}\left(K_{U} / K, E_{l^{n}}\right)^{*}
$$

where $N^{*}$, for a $\mathbb{Z} / l^{n} \mathbb{Z}$-module $N$, denotes $\operatorname{Hom}\left(N, \mathbb{Z} / l^{n} \mathbb{Z}\right)$ and where $K_{U}$ is the maximal extension of $K$ unramified outside $U_{K}$. Hence, the image $I$ of $H^{1}\left(K_{U} / K, E_{l^{n}}\right)$ in $\bigoplus_{v \in U_{K}} H^{1}\left(K_{v}, E_{l^{n}}\right)$ is a maximal isotropic subgroup, for the Tate pairing, of the group

$$
\bigoplus_{v \in U_{K}} H^{1}\left(K_{v}, E_{l^{n}}\right)
$$

Since at the place $y$ lying over $z$ we have $H^{1}\left(K_{y}, E_{l^{n}}\right)^{\delta} \neq 0$ by Lemma 5.1.4(a), the subgroup $I^{\delta}$ is of order strictly larger than that of

$$
\bigoplus_{u \in S} \frac{H^{1}\left(K_{u^{\sharp}}, E_{l^{n}}\right)^{\delta}}{H_{u^{\sharp}}} .
$$

Hence, the natural homomorphism

$$
H^{1}\left(K_{U} / K, E_{l^{n}}\right)^{\delta} \rightarrow \bigoplus_{u \in S} \frac{H^{1}\left(K_{u^{\sharp}}, E_{l^{n}}\right)^{\delta}}{H_{u^{\sharp}}}
$$

has nonzero kernel. Therefore, we may select a nonzero element $h \in H^{1}\left(K_{U} / K, E_{l^{n}}\right)^{\delta}$ in this kernel. Then $h$ satisfies condition (b); that is to say, we have $h_{u^{\sharp}} \in H_{u^{\sharp}}$ for all $u \in S$. Furthermore, $h$ satisfies condition (a) by the selection of $H_{v}$ if $v \in \Sigma_{K}$ is a bad reduction place of $E$ and because we have $H^{1}\left(K_{v}^{\text {un }} / K_{v}, E_{l^{n}}\right)=\partial_{l^{n}}\left(E\left(K_{v}\right)\right)$ if $v \in \Sigma_{K}$ is a good reduction place of $E$, where $K_{v}^{\text {un }}$ is the maximal unramified separable extension of $K_{v}$.

REMARK 5.1.6

Lemma 5.1.5 is a technical result required for the proof of Proposition 5.2.1 in the next section.

\subsection{A class $\gamma_{n}(c)$ in the Selmer group}

The notation and hypotheses of Section 5.1.1 also hold for this section. 
PROPOSITION 5.2.1

Let $r \geq 1$ be an integer, and put $\nu(r)=(-1)^{r} \epsilon$. Let $G$ be a subgroup of the Selmer eigenspace $\operatorname{Sel}_{l \infty}(E / K)^{-\nu(r)}$ such that

$$
\operatorname{rank}(G) \leq r .
$$

Assume that $M_{r-1}$ is finite and that $l \in \mathcal{P}$ is coprime to the order of $\operatorname{Pic}(A)$. Then there is a cohomology class $\gamma_{M_{r-1}}(c)$ for some divisor $c \in \Lambda^{r}\left(M_{r-1}\right)$ such that

(a) $\gamma_{M_{r-1}}(c)$ belongs to the Selmer eigenspace $\operatorname{Sel}_{l \infty}(E / K)^{-\nu(r)}$;

(b) $\gamma_{M_{r-1}}\left(\right.$ c) has order $l^{M_{r-1}-M_{r}}$;

(c) $\mathbb{Z} \gamma_{M_{r-1}}(c) \cap G=\{0\}$.

Proof

The group $\operatorname{Sel}_{l \infty}(E / K)$ is an $l^{\infty}$-torsion group; that is to say, every element is annihilated by a power of $l$. Hence, the subgroup $G$ of finite rank is finite.

Let $\exp (G)$ be the exponent of $G$, that is to say, the largest order of an element of the abelian $l$-group $G$. Select an integer

$$
m \geq 1
$$

such that

$$
l^{m} \geq \max \left\{\exp (G), l^{M_{r-1}}\right\},
$$

and put

$$
L=K\left(E_{l^{m}}\right)
$$

Put

$$
t=\operatorname{rank}(G)
$$

where

$$
\operatorname{rank}(G)=\operatorname{dim}_{\mathbb{Z} / l \mathbb{Z}} G / l G
$$

and where $t \leq r$ by hypothesis. Note that $G$ is a subgroup of $\operatorname{Hom}\left(\operatorname{Gal}\left(L^{\mathrm{sep}} / L\right)\right.$, $E_{l^{m}}(L)$ ), as $l^{m} \geq \exp (G)$ (see Lemma 3.3.1), and the elements of $\Lambda^{1}(m)$ are unramified in $L / F$. As in Section 1.2, for any divisor $c$ on $F, \operatorname{Supp}(c)$ denotes the set of distinct prime divisors in the support of $c$.

For any divisor $c$ in $\Lambda^{r}(1)$, put

$$
\Xi(c)=\operatorname{Supp}(c) \cap \Lambda^{1}(m) ;
$$

that is to say, $\Xi(c)$ is the set of prime divisors in the support of $c$ which belong to $\Lambda^{1}(m)$. In particular, $\Xi(c)$ depends on $m$. For any prime divisor $z$ of $F$ in $\Lambda^{1}(1)$, select a place $z^{\times}$of $L=K\left(E_{l^{m}}\right)$ lying over $z$. Let $\Gamma(c) \subseteq \widehat{G}$ be the subgroup of characters of the abelian group $G$ generated by the set of characters (as in Proposition 3.3.2 and (3.3.1), applied to the finite group $G$, and Proposition 3.3.3)

$$
\left\{\phi_{\operatorname{Frob}\left(z^{\times}\right)} \mid z \in \Xi(c)\right\} .
$$


Put

$$
s(c)=\operatorname{dim}_{\mathbb{Z} / l \mathbb{Z}} \frac{\Gamma(c)+l \widehat{G}}{l \widehat{G}} .
$$

That is to say, $s(c)$ is the dimension of the image of $\Gamma(c)$ in the vector space $\widehat{G} / l \widehat{G}$ of dimension $t$; the nonnegative integer $s(c)$ is then at most equal to $t$. Put

$$
n(c)=|\Xi(c)|
$$

that is to say, $n(c)$ is the cardinality of $\Xi(c)$ and is a nonnegative integer at most equal to $r$, the number of prime divisors in the support of $c$.

Define the defect $\Delta(c)$ of a divisor $c \in \Lambda^{r}(1)$ on $F$ to be

$$
\Delta(c)=\max (t-s(c), r-n(c)) .
$$

Then we have

$$
0 \leq \Delta(c) \leq r
$$

and we have

$$
\Delta(c)=0 \quad \text { if and only if } \Gamma(c)=\widehat{G} \text { and } c \in \Lambda^{r}(m) .
$$

This equivalence holds because $s(c)=t$ if and only if $\Gamma(c)=\widehat{G}$ by Nakayama's lemma.

We have that $M_{r-1} \geq M_{r}$ by Lemma 5.1.2, and in particular, $M_{r}$ is finite as $M_{r-1}$ is assumed to be finite. We may then select a divisor

$$
d \in \Lambda^{r}\left(M_{r}+1\right)
$$

such that

$$
l^{M_{r}} \| P_{d}
$$

Then the cohomology class $\gamma_{M_{r}+1}(d)$ is defined, belongs to $H^{1}\left(K, E_{l^{M_{r}+1}}\right)^{-\nu(r)}$, and has exact order $l$ by Lemma 4.2.2 and Proposition 3.4.1(a).

Suppose that $M_{r}=M_{r-1}$. Then $\gamma_{M_{r-1}}(d)$ is equal to zero and evidently belongs to the Selmer eigenspace $\operatorname{Sel}_{l \infty}(E / K)^{-\nu(r)}$, which proves the lemma in this trivial case where $M_{r}=M_{r-1}$. We may suppose for the rest of the proof of this proposition that $M_{r-1}>M_{r}$.

Assume that the defect of the divisor $d$ already selected in (5.2.1) satisfies

$$
\Delta(d)>0 .
$$

That is to say, either (where $s(d)<t$ ) the image of $\Gamma(d)$ is a proper subspace of $\widehat{G} / l \widehat{G}$ or (where $n(d)<r) d \notin \Lambda^{r}(m)$. These two possibilities that $s(d)<t$ and that $n(d)<r$ for the divisor $d$ are not mutually exclusive.

We select a character $\psi \in \widehat{G}$ and a prime divisor $z_{0} \in \operatorname{Supp}(d)$ with the following recipe.

Selection of the character $\psi$.

If $s(d)<t$, then select a character $\psi \in \widehat{G}$ such that

$$
\psi\left(\gamma_{M_{r}+1}(d)\right) \neq 0 \quad \text { if } \gamma_{M_{r}+1}(d) \in G
$$


and

$$
\psi \in \widehat{G} \backslash(\Gamma(d)+l \widehat{G}),
$$

where the condition (5.2.2) is vacuous if $\gamma_{M_{r}+1}(d) \notin G$. A character $\psi \in \widehat{G}$ exists which satisfies the two conditions (5.2.2) and (5.2.3) because a finite group cannot be the union of two proper subgroups.

If $s(d)=t$ and $n(d)<r$, then select any character $\psi \in \widehat{G}$ such that

$$
\psi\left(\gamma_{M_{r}+1}(d)\right) \neq 0 \quad \text { if } \gamma_{M_{r}+1}(d) \in G,
$$

where the condition (5.2.4) is vacuous if $\gamma_{M_{r}+1}(d) \notin G$.

Selection of the divisor $z_{0} \in \operatorname{Supp}(d)$.

If $s(d)<t$, then select a prime divisor $z_{0} \in \operatorname{Supp}(d)$ such that

$$
\left\{\phi_{\operatorname{Frob}\left(z^{\times}\right)} \mid z \in \Xi\left(d-z_{0}\right)\right\}
$$

is a generating set for $\Gamma(d)$ modulo $l \widehat{G}$. This choice is possible because $t \leq r$ and $\operatorname{Supp}(d)$ has $r$ distinct elements.

If $s(d)=t$ and $n(d)<r$, then select a prime divisor $z_{0} \in \operatorname{Supp}(d)$ such that $z_{0} \notin \Lambda^{1}(m)$.

We have now defined the pair $\psi, z_{0}$ for the divisor $d$ where the defect $\Delta(d)>0$. By Lemma 5.1.5 we may select a cohomology class $h$ in the $\nu(r)$-eigenspace

$$
h \in H^{1}\left(K, E_{l}\right)^{\nu(r)}
$$

of the group scheme $E_{l}$ such that

(5.2.5) $h \neq 0$;

(5.2.6) $h_{v} \in \partial_{l}\left(E\left(K_{v}\right)\right)$

for all places $v$ of $K$ coprime to $\operatorname{Supp}(d)$; and

(5.2.7) $h_{y} \in \operatorname{Im}\left(\chi_{z}\right)$

for all $z \in \operatorname{Supp}\left(d-z_{0}\right)$ where $y$ is the prime of $K$ lying over $z$ and $\chi_{z}$ is the homomorphism of Section 4.4.

Note that $H^{1}\left(K, E_{l}\right)^{\nu(r)}$ is a subgroup of $H^{1}\left(K, E_{l^{m}}\right)$ by Lemma 3.1.4.

Since $h$ is in a different eigenspace from $G$ and $\gamma_{M_{r}+1}(d)$, which both belong to the $-\nu(r)$-eigenspace, we have that

$$
\left(G+\mathbb{Z} \gamma_{M_{r}+1}(d)\right) \cap \mathbb{Z} h=0,
$$

where $G+\mathbb{Z} \gamma_{M_{r}+1}(d)$ is the subgroup of $H^{1}\left(K, E_{l^{m}}\right)^{-\nu(r)}$ generated by $G$ and $\gamma_{M_{r}+1}(d)$ and where $\mathbb{Z} h$ is the subgroup of $H^{1}\left(K, E_{l^{m}}\right)^{\nu(r)}$ generated by $h$.

Let $D$ be the subgroup of $H^{1}\left(K, E_{l^{m}}\right)$ generated by $G, \gamma_{M_{r}+1}(d)$, and $h$,

$$
D=G+\mathbb{Z} \gamma_{M_{r}+1}(d)+\mathbb{Z} h .
$$

Then $D$ is a finite subgroup of $H^{1}\left(K, E_{l^{m}}\right)$ by Lemma 3.1.4. As $D$ is isomorphic to the direct product of $G+\mathbb{Z} \gamma_{M_{r}+1}(d)$ and $\mathbb{Z} h$, we can select a homomorphism

$$
\chi: D \rightarrow \mathbb{Q} / \mathbb{Z}
$$


such that (by (5.2.2), (5.2.4), and (5.2.5))

$$
\begin{aligned}
\left.\chi\right|_{G} & =\psi ; \\
\chi\left(\gamma_{M_{r}+1}(d)\right) & \neq 0 ; \\
\chi(h) & \neq 0 .
\end{aligned}
$$

By Proposition 3.3.3 applied to the finite group $D$, there is a prime divisor

$$
z_{1} \in \Lambda^{1}(m)
$$

distinct from the elements of $\operatorname{Supp}(d)$ such that, in the notation of (3.3.1),

$$
\chi=\phi_{\operatorname{Frob}\left(z_{1}^{\times}\right)},
$$

where $z_{1}^{\times}$is a prime divisor of $L=K\left(E_{l^{m}}\right)$ above $z_{1}$. We then obtain the cohomology class $\gamma_{M_{r}+1}\left(d+z_{1}\right)$ associated to the divisor $d+z_{1}$.

For all places $v$ of $K$, denote the alternating cup product induced by the Weil pairing of $h$ with $\gamma_{M_{r}+1}\left(d+z_{1}\right)$ localized at $v$ by (see Theorem 2.1.1)

$$
\left\langle\gamma_{M_{r}+1}\left(d+z_{1}\right)_{v}, h_{v}\right\rangle_{v}
$$

which is an element of $\mathbb{Q} / \mathbb{Z}$. The sum of local pairings over all places of $K$

$$
\sum_{\text {all places } v \text { of } K}\left\langle\gamma_{M_{r}+1}\left(d+z_{1}\right)_{v}, h_{v}\right\rangle_{v}=0
$$

is zero by Proposition 2.1.3.

If $v$ does not divide any element of $\operatorname{Supp}\left(d+z_{1}\right)$, then

$$
\gamma_{M_{r}+1}\left(d+z_{1}\right)_{v} \in \partial_{l^{M_{r}+1}}\left(E\left(K_{v}\right)\right)
$$

by Proposition 4.5.1(a) and

$$
h_{v} \in \partial_{l}\left(E\left(K_{v}\right)\right)
$$

by (5.2.6); that is to say, both localized elements $\gamma_{M_{r}+1}\left(d+z_{1}\right)_{v}$ and $h_{v}$ are in the image of the map

$$
\partial_{l^{m}}: E\left(K_{v}\right) \rightarrow H^{1}\left(K_{v}, E_{l^{m}}\right) .
$$

But the image of this map $\partial_{l^{m}}$ in $H^{1}\left(K_{v}, E_{l^{m}}\right)$ is an isotropic subgroup for the alternating pairing $\langle\cdot, \cdot\rangle_{v}$ (see [1, Theorem 7.15.6, p. 403] or Theorem 2.1.2(a)). Therefore, we have that

$$
\left\langle\gamma_{M_{r}+1}\left(d+z_{1}\right)_{v}, h_{v}\right\rangle_{v}=0 \text { for all } v \text { coprime to } \operatorname{Supp}\left(d+z_{1}\right) .
$$

If $y$ is a place of $K$ which divides an element $z$ of $\operatorname{Supp}\left(d-z_{0}\right)$, then we have $h_{y} \in \operatorname{Im}\left(\chi_{z}\right)$ by $(5.2 .7)$ and

$$
\gamma_{M_{r}+1}\left(d+z_{1}\right)_{y} \in \operatorname{Im}\left(\chi_{z}\right)
$$

by Proposition 4.4.2(d), which is the main property of the map $\chi_{z}$. As $\operatorname{Im}\left(\chi_{z}\right)$ is isotropic for the cup product $\langle\cdot, \cdot\rangle_{v}$ by Lemma 5.1.4(b), we have that

$$
\left\langle\gamma_{M_{r}+1}\left(d+z_{1}\right)_{y}, h_{y}\right\rangle_{y}=0 \text { for all } y \text { dividing an element of } \operatorname{Supp}\left(d-z_{0}\right) .
$$


Therefore, the only possible nonzero terms in the $\operatorname{sum} \sum_{v}\left\langle\gamma_{M_{r}+1}\left(d+z_{1}\right)_{v}, h_{v}\right\rangle_{v}$ of (5.2.13) are for the places of $K$ lying over the places $z_{0}$ and $z_{1}$. For these places $z_{i} \in \Lambda^{1}$ of $F$, for $i=0,1$, denote by $y_{i}$ the corresponding place of $K$ lying over the place $z_{i}$ which remains inert in $K / F$.

From (5.2.9) and Section 3.3.2 and that $\chi=\phi_{\operatorname{Frob}\left(z_{1}^{\times}\right)}$by (5.2.12), we have that this localization $\gamma_{M_{r}+1}(d)_{y_{1}}$ at $y_{1}$ is nonzero; hence by Proposition 4.5.1(d) the localization

$$
\delta_{M_{r}+1}\left(d+z_{1}\right)_{y_{1}} \in H^{1}\left(K_{y_{1}}, E\right)_{l^{M_{r}+1}}^{\nu(r)}
$$

is nonzero. Furthermore, we have that

$$
h_{y_{1}} \in \partial_{l}\left(E\left(K_{y_{1}}\right)\right)^{\nu(r)}
$$

by (5.2.6) and $h_{y_{1}}$ is nonzero by (5.2.10) and (5.2.12). Hence, we have by Lemma 5.1.4(a) that

$$
\left\langle\gamma_{M_{r}+1}\left(d+z_{1}\right)_{y_{1}}, h_{y_{1}}\right\rangle_{y_{1}} \neq 0 .
$$

Since the sum $\sum_{v}\left\langle\gamma_{M_{r}+1}\left(d+z_{1}\right)_{v}, h_{v}\right\rangle_{v}$ of (5.2.13) is zero, this implies that the local term at $y_{0}$

$$
\left\langle\gamma_{M_{r}+1}\left(d+z_{1}\right)_{y_{0}}, h_{y_{0}}\right\rangle_{y_{0}}
$$

is nonzero. Hence, we have $\gamma_{M_{r}+1}\left(d+z_{1}\right)_{y_{0}} \neq 0$ and so by Proposition 4.5.1(d)

$$
P_{d+z_{1}-z_{0}} \notin l^{M_{r}+1} E\left(K_{y_{0}}\right) \text {. }
$$

Hence we obtain, where $z_{1} \in \Lambda^{1}(m)$,

$$
l^{M_{r}} \| P_{d+z_{1}-z_{0}}
$$

because we have $l^{M_{r}} \mid P_{d+z_{1}-z_{0}}$ and $d+z_{1}-z_{0} \in \Lambda^{r}\left(M_{r}+1\right)$ as well as $d \in$ $\Lambda^{r}\left(M_{r}+1\right)$ and $z_{1} \in \Lambda^{1}(m)$.

Put

$$
c_{1}=d+z_{1}-z_{0} \in \Lambda^{r}\left(M_{r}+1\right) .
$$

On the one hand, we have that if $s(d)<t$, then the group $\Gamma\left(c_{1}\right)$ is generated by $\Gamma(d)$ and $\psi$,

$$
\Gamma\left(c_{1}\right)=\Gamma(d)+\mathbb{Z} \psi,
$$

because $\chi=\phi_{\operatorname{Frob}\left(z_{1}^{\times}\right)}$by (5.2.12) and $\left.\chi\right|_{G}=\psi$ by (5.2.8) in this case where $s(d)<t$. The condition that $z_{1} \in \Lambda(m)$ is satisfied by the choice of $z_{1}$ in (5.2.11). We then have from (5.2.15) if $s(d)<t$, as $\psi \notin \Gamma(d)+l \widehat{G}$ by (5.2.3),

$$
s\left(c_{1}\right)=\operatorname{dim}_{\mathbb{Z} / l \mathbb{Z}} \frac{\Gamma\left(c_{1}\right)+l \widehat{G}}{l \widehat{G}}=s(d)+1 .
$$

On the other hand, if $s(d)=t$ and $n(d)<r$, then $n\left(c_{1}\right)=n(d)+1$ as $z_{1} \in$ $\Lambda^{1}(m)$ by $(5.2 .11)$ and $z_{0} \notin \Lambda^{1}(m)$ by the selection of $z_{0} \in \operatorname{Supp}(d)$. Hence, we have in both cases that the defect of $c_{1}$ is given by

$$
\Delta\left(c_{1}\right)=\Delta(d)-1 \text {. }
$$


We may by this method construct by induction a sequence of divisors $c_{1}, c_{2}, c_{3}, \ldots$ in $\Lambda^{r}\left(M_{r}+1\right)$ such that their defects are strictly decreasing

$$
\Delta(d)>\Delta\left(c_{1}\right)>\Delta\left(c_{2}\right)>\cdots
$$

and where, as in (5.2.14),

$$
l^{M_{r}} \| P_{c_{i}} \text { for all } i \text {. }
$$

This sequence must terminate in a divisor $c$ with zero defect $\Delta(c)=0$, that is to say, $\Gamma(c)=\widehat{G}$, by Nakayama's lemma, and $c \in \Lambda^{r}(m)$ where

$$
l^{M_{r}} \| P_{c}
$$

The cohomology class $\gamma_{m}(c)$ is therefore defined, and as $\Gamma(c)=\widehat{G}$ we have, where $z^{\times}$is a place of $K\left(E_{l^{m}}\right)$ over $z$,

$$
\left\{g \in G \mid \phi_{\operatorname{Frob}\left(z^{\times}\right)}(g)=0 \text { for all } z \in \operatorname{Supp}(c)\right\}=0 .
$$

We have from Lemma 5.1.3(b) that

$$
\gamma_{M_{r-1}}(c) \in \operatorname{Sel}_{l \infty}(E / K)^{-\nu(r)} .
$$

We have by the definition of $M_{r-1}$ that $\gamma_{M_{r-1}}(c-z)=0$ for any prime divisor $z$ in the support of $c$ as $c-z$ has $r-1$ elements in its support. But by Proposition 4.5.1(d), we have for any $z \in \operatorname{Supp}(c)$, where $y$ is the place of $K$ over $z$,

$$
\operatorname{ord} \gamma_{M_{r-1}}(c)_{y}=\operatorname{ord} \gamma_{M_{r-1}}(c-z)_{y} \text {. }
$$

Hence we obtain for any $z \in \operatorname{Supp}(c)$ that $\gamma_{M_{r-1}}(c)_{y}=0$ where $y$ is the prime of $K$ over $z$. We then obtain from (5.2.18), as $g_{y}=\phi_{\operatorname{Frob}\left(z^{\times}\right)}(g)$ where $y$ is the place of $K$ above $z$, that

$$
G \cap \mathbb{Z} \gamma_{M_{r-1}}(c)=0 .
$$

Since $l^{M_{r}} \| P_{c}$, by (5.2.17), we have that $\gamma_{m}(c)$ has order $l^{m-M_{r}}$ for all $m \geq M_{r}$ by Proposition 3.4.1(a). Hence, $\gamma_{M_{r-1}}(c)$ has order $l^{M_{r-1}-M_{r}}$ and belongs to the Selmer group $\operatorname{Sel}_{l \infty}(E / K)^{-\nu(r)}$, by (5.2.19), which proves the proposition.

\subsection{Proof of Theorem 4.1 .10}

\subsection{1.}

The notation from Section 5.1.1 also holds for this section.

LEMMA 5.3.1

Let $A$ be a finite abelian $p$-group where $p$ is a prime number and with invariants $I_{1} \geq I_{2} \geq \cdots \geq I_{r}$. Let $B$ be a subgroup of $A$ with invariants $I_{1} \geq I_{2} \geq \cdots \geq I_{s}$ where $s \leq r$. Then there is a subgroup $C$ of $A$ such that

$$
A=B \oplus C ;
$$

that is to say, $A$ is the direct sum of the subgroups $B, C$. The invariants of $C$ are $I_{s+1} \geq I_{s+1} \geq \cdots \geq I_{r}$. 
The proof of this result follows from the structure theorem of finite abelian groups and is omitted.

Fof the proof of Theorem 4.1.10, as $P_{0}$ has infinite order in $E(K)$, the TateShafarevich group $\amalg(E / K)$ is finite and $P_{0}$ generates a subgroup of finite index in $E(K)$ by [1, Theorem 7.6.5], which is one of the principal results of [1].

The image of $P_{0}$ in $l^{m} E(K)$ belongs to the $-\epsilon$-eigenspace of $l^{m} E(K)$ for all $m$ (by [1, Lemma 7.14.11] or Lemma 4.2.2 above). It follows from the decomposition into eigenspaces

$$
l^{m} E(K) \cong\left(l^{m} E(K)\right)^{\epsilon} \oplus\left({ }_{l^{m}} E(K)\right)^{-\epsilon}
$$

that the $\epsilon$-eigenspace $\left(l^{m} E(K)\right)^{\epsilon}$ is a finite abelian group of order bounded independently of $m$, and hence, $E(K)^{\epsilon}$ is a finite abelian group. As $E(K)$ has no $l$-torsion (as $l \in \mathcal{P}$; see Proposition 1.10.1 and Definition 3.1.2(f)), it follows that

$$
\left({ }_{l^{m}} E(K)\right)^{\epsilon}=0 \text { for all } m
$$

and that

$$
\left({ }_{l^{m}} E(K)\right)^{-\epsilon} \cong \mathbb{Z} / l^{m} \mathbb{Z} \text { for all } m,
$$

which proves the isomorphisms (4.1.3).

The $l^{m}$-Selmer group $\operatorname{Sel}_{l^{m}}(E / K)^{ \pm}$belongs to an exact sequence of eigenspaces

$$
0 \longrightarrow\left({ }_{l^{m}} E(K)\right)^{ \pm} \longrightarrow \operatorname{Sel}_{l^{m}}(E / K)^{ \pm} \longrightarrow \amalg(E / K)_{l^{m}}^{ \pm} \longrightarrow 0 .
$$

Hence, this exact sequence induces an isomorphism of $\epsilon$-eigenspaces

$$
\operatorname{Sel}_{l \infty}(E / K)^{\epsilon} \cong \amalg(E / K)_{l^{\infty}}^{\epsilon} .
$$

The largest invariant of the $-\epsilon$-eigenspace $\operatorname{Sel}_{l^{m}}(E / K)^{-\epsilon}$ is at most equal to $m$. But this group $\operatorname{Sel}_{l^{m}}(E / K)^{-\epsilon}$ contains the subgroup $\left(l^{m} E(K)\right)^{-\epsilon} \cong \mathbb{Z} / l^{m} \mathbb{Z}$ with invariant $m$. By Lemma 5.3.1 applied to the eigenspace $\operatorname{Sel}_{l^{m}}(E / K)^{-\epsilon}$ and the subgroup $\left(l_{m} E(K)\right)^{-\epsilon}$, we have that the $-\epsilon$-eigencomponent of the exact sequence (5.3.3) splits. Hence, we obtain an isomorphism of $-\epsilon$-eigenspaces

$$
\operatorname{Sel}_{l^{m}}(E / K)^{-\epsilon} \cong{ }_{l^{m}} E(K) \oplus \amalg(E / K)_{l^{m}}^{-\epsilon} \text { for all } m .
$$

The isomorphisms (5.3.4) and (5.3.5) prove the theorem.

\subsection{Proofs of Theorems 4.1 .4 and 4.1 .8}

\subsection{1.}

The principle of the proof is to construct inductively divisors $c_{k} \in \Lambda^{k}, k=$ $1,2, \ldots$, such that the cohomology classes $\delta_{M_{k-1}}\left(c_{k}\right)$ in the Tate-Shafarevich group $\amalg(E / K)_{l^{\infty}}$ form a basis of a maximal isotropic subgroup with respect to the Cassels pairing and where $\delta_{M_{k-1}}\left(c_{k}\right)$ has order $l^{N_{k}}$ for all $k$. The inductive step is provided by Lemma 5.4.1. 


\subsection{2.}

The notation and hypotheses from Section 5.1.1 also hold for this section. We further denote by, where $l \in \mathcal{P}$,

$\Sigma_{K}$ the set of all places of the global field $K$;

$[\cdot, \cdot]_{v}: l^{m} E\left(K_{v}\right) \times H^{1}\left(K_{v}, E\right)_{l^{m}} \rightarrow \mathbb{Z} / l^{m} \mathbb{Z}$ the nondegenerate local pairing at the place $v$ of $K$ induced by the cup product, as in Theorem 2.1.2.

If $z_{i} \in \Lambda^{1}(1)$ is a prime divisor of $F$ indexed by an integer $i$, then denote by $y_{i}$ the prime divisor of $K$ lying above the place $z_{i}$ of $F$ where this place $z_{i}$ is inert in the field extension $K / F$.

\section{LEMMA 5.4.1}

Assume that $l \in \mathcal{P}$ is coprime to the order of $\operatorname{Pic}(A)$. Let $s \geq 1$ be a positive integer, and let $r, t \geq 0$ be nonnegative integers. Let $S$ be a subgroup of $\operatorname{Sel}_{l^{s}}(E / K)$. Let $e \in \operatorname{Sel}_{l^{s}}(E / K)^{-\nu(r+1)}$ and $\gamma_{s}(c) \in \operatorname{Sel}_{l^{s}}(E / K)^{-\nu(r)}$, where $c \in \Lambda^{r}(s+t)$, be elements of the Selmer group where

$$
S \cap\left(e, \gamma_{s}(c)\right)=0
$$

and where $\left(e, \gamma_{s}(c)\right)$ is the subgroup of the Selmer group generated by e and $\gamma_{s}(c)$. Suppose also that e and $\gamma_{s}(c)$ both have order $l^{n}$ where $n \leq s$. Then there are infinitely many prime divisors $z \in \Lambda^{1}(s+t)$ coprime to $\operatorname{Supp}(c)$ such that if $y$ is the place of $K$ over $z$, then we have that

(a) $S_{y}=0$;

(b) the value in $\mathbb{Z} / l^{n} \mathbb{Z}$ of the local pairing at $y$ with the class $\delta_{s}(c+z)$

$$
\left[\delta_{s}(c+z)_{y}, w_{y}\right]_{y}
$$

has order $l^{n}$ where $w_{y} \in l_{l^{n}} E\left(K_{y}\right)$ is a point such that $e_{y}=\partial_{l^{n}}\left(w_{y}\right)$.

Proof

Note that the isomorphism from (3.1.3), we have that $\operatorname{Sel}_{l^{n}}(E / K)$ is the subgroup of $\operatorname{Sel}_{l^{s}}(E / K)$ annihilated by $l^{n}$, and in particular, $\operatorname{Sel}_{l^{n}}(E / K)$ contains $e$ and $\gamma_{s}(c)$.

Let $T$ be the subgroup of the Selmer group $\operatorname{Sel}_{l^{s}}(E / K)$ generated by $S, e$, and $\gamma_{s}(c)$. Then we have an isomorphism

$$
T \cong S \oplus\left(e, \gamma_{s}(c)\right) .
$$

For a fixed nonzero element $x \in T$, the set of characters

$$
\chi: T \rightarrow \mathbb{Z} / l^{s} \mathbb{Z}
$$

such that

$$
\operatorname{ord}(\chi(x))<\operatorname{ord}(x)
$$

is a proper subgroup of $\widehat{T}$. This follows as the subgroups of $\mathbb{Z} / l^{s} \mathbb{Z}$ are linearly ordered $\mathbb{Z} / l^{s} \mathbb{Z} \supseteq l \mathbb{Z} / l^{s} \mathbb{Z} \supseteq l^{2} \mathbb{Z} / l^{s} \mathbb{Z} \supseteq \cdots$. As a group cannot be the union of two 
proper subgroups, there is then a character $\chi: T \rightarrow \mathbb{Z} / l^{s} \mathbb{Z}$ such that

$$
\begin{aligned}
\operatorname{ord}(\chi(e)) & =\operatorname{ord}(e), \\
\operatorname{ord}\left(\chi\left(\gamma_{s}(c)\right)\right) & =\operatorname{ord}\left(\gamma_{s}(c)\right), \\
\chi(S) & =0 .
\end{aligned}
$$

By Proposition 3.3.4 applied to the subgroup $T$ and the character $\chi$, we may select a prime divisor $z \in \Lambda^{1}(s+t)$ satisfying, where $y$ is the place of $K$ lying over $z$,

$$
\begin{aligned}
\operatorname{ord}\left(e_{y}\right) & =\operatorname{ord}(e), \\
\operatorname{ord}\left(\gamma_{s}(c)_{y}\right) & =\operatorname{ord}\left(\gamma_{s}(c)\right), \\
S_{y} & =0,
\end{aligned}
$$

where the subscript $y$ denotes the restriction at $y$ of elements of the Selmer group $\operatorname{Sel}_{l^{s}}(E / K)$. In particular, condition (a) of the lemma is satisfied for this $z$. The class $\delta_{s}(c+z)$, associated to the divisor $c+z \in \Lambda^{r+1}(s+t)$, then belongs to $H^{1}(K, E)_{l^{s}}^{-\nu(r+1)}$ and $e$ belongs to $H^{1}\left(K, E_{l^{s}}\right)^{-\nu(r+1)}$.

We may select a point $w_{y} \in\left(l^{n} E\left(K_{y}\right)\right)^{-\nu(r+1)}$ such that $e_{y}=\partial_{l^{n}}\left(w_{y}\right)$ where $e \in \operatorname{Sel}_{l^{n}}(E / K)$ as already noted. Then $w_{y}$ has order $l^{n}$ in $l^{n} E\left(K_{y}\right)$ as $e_{y}$ has order $l^{n}$.

Furthermore, because

$$
\operatorname{ord}\left(\gamma_{s}(c)_{y}\right)=\operatorname{ord}\left(\gamma_{s}(c)\right)
$$

and by Proposition 4.5.1(d) we must have that

$$
\operatorname{ord}\left(\delta_{s}(c+z)_{y}\right)=\operatorname{ord}\left(\gamma_{s}(c)\right)=l^{n} .
$$

The class $\delta_{s}(c+z)_{y}$, of order $l^{n}$, belongs to the subgroup $H^{1}\left(K_{y}, E\right)_{l^{n}}^{-\nu(r+1)}$ of $H^{1}\left(K_{y}, E\right)_{l^{s}}^{-\nu(r+1)}$, and $w_{y}$, which is of order $l^{n}$, belongs to $\left({ }_{l^{n}} E\left(K_{y}\right)\right)^{-\nu(r+1)}$. In particular, $\delta_{s}(c+z)$ and $w_{y}$ both belong to the $-\nu(r+1)$-eigenspaces of their respective spaces. Hence, the local term

$$
\left[\delta_{s}(c+z)_{y}, w_{y}\right]_{y}
$$

has order $l^{n}$. This follows from the nondegeneracy of the local pairing: by Lemma 5.1.4(a), if $z \in \Lambda^{1}(n)$ and $y \in \Sigma_{K}$ is the place of $K$ over $z$, then the two elements

$$
f \in l^{n} E\left(K_{y}\right), \quad d \in H^{1}\left(K_{y}, E\right)_{l^{n}}
$$

give via the Tate pairing an element

$$
[f, d]_{y}
$$

which is nonzero if they are in the same $\tau$-eigenspace and the product of their orders is greater than or equal to $l^{n}$. Therefore, condition (b) is satisfied by $z$, which proves the lemma. 
We now simultaneously prove Theorems 4.1.4 and 4.1.8.

As $P_{0}$ has infinite order in $E(K)$, the Tate-Shafarevich group $\amalg(E / K)$ is finite and $P_{0}$ generates a subgroup of finite index in $E(K)$ by [1, Theorem 7.6.5]. By the definition of the invariants $N_{i}$ of the finite abelian group $\amalg(E / K)_{l^{\infty}}$ in Section 4.1.5, there is a maximal isotropic subgroup $D$ of $\amalg(E / K)_{l \infty}$, with respect to the nondegenerate antisymmetric Cassels pairing, where

$$
D=\prod_{i} D_{i}
$$

each $D_{i}$ is a cyclic group of order $l^{N_{i}}$,

$$
D^{\epsilon}=\prod_{i \text { odd }} D_{i}
$$

and

$$
D^{-\epsilon}=\prod_{i \text { even }} D_{i}
$$

From Theorem 4.1.10, we have the decomposition of eigenspaces

$$
\operatorname{Sel}_{l^{m}}(E / K)^{ \pm} \cong\left(l^{m} E(K)\right)^{ \pm} \oplus \amalg(E / K)_{l^{m}}^{ \pm} \text {for all } m \geq 0,
$$

where the projection onto the second factor is given by the natural surjection

$$
\pi_{m}: \operatorname{Sel}_{l^{m}}(E / K) \longrightarrow \amalg(E / K)_{l^{m}} .
$$

Let $m$ be an integer such that $l^{m}$ is greater than or equal to the exponent of the finite group $\amalg(E / K)_{l}$; that is to say, $m \geq \max _{i} N_{i}$. For each integer $i$, let $d_{i} \in \amalg(E / K)_{l^{\infty}}^{-\nu(i)}$ be a generator of $D_{i}$, and let

$$
e_{i} \in \operatorname{Sel}_{l^{m}}(E / K)^{-\nu(i)}, \quad \text { where } \pi_{m}\left(e_{i}\right)=d_{i}, \operatorname{ord}\left(e_{i}\right)=l^{N_{i}},
$$

be the lifting of $d_{i}$ to the Selmer eigenspace $\operatorname{Sel}_{l^{m}}(E / K)^{-\nu(i)}$ via the decomposition (5.4.1) such that $e_{i}$ has zero component in the subgroup $l^{m} E(K)$; in particular, we take $e_{i}$ to have order equal to $l^{N_{i}}$ for all $i$ and to belong to the $-\nu(i)$-eigenspace as $d_{i}$ has order $l^{N_{i}}$. For each valuation $v$ of $K$ and each $i$, select $w_{i, v} \in_{l^{N_{i}}} E\left(K_{v}\right)$ such that the localization $e_{i, v}$ of $e_{i}$ at $v$ satisfies

$$
e_{i, v}=\partial_{l^{N_{i}}}\left(w_{i, v}\right) \text {. }
$$

Here

$$
\partial_{l^{N_{i}}}:{ }_{l^{N_{i}}} E\left(K_{v}\right) \rightarrow H^{1}\left(K_{v}, E_{l^{N_{i}}}\right)
$$

denotes the connecting homomorphism associated to the morphism $l^{N_{i}}: E \rightarrow E$ of multiplication by $l^{N_{i}}$.

The cohomology class $\gamma_{M_{0}+N_{1}}(0)$ belongs to the Selmer group $\operatorname{Sel}_{l^{m}}(E / K)^{-\epsilon}$ and has order $l^{N_{1}}$ as $l^{M_{0}} \| P_{0}$, by Lemma 5.1.1 or Proposition 3.4.1(a),

$$
\operatorname{ord}\left(\gamma_{M_{0}+N_{1}}(0)\right)=l^{N_{1}} .
$$

The element $e_{1} \in \operatorname{Sel}_{l^{m}}(E / K)^{\epsilon}$ has the same order

$$
\operatorname{ord}\left(e_{1}\right)=l^{N_{1}} \text {. }
$$


Let $S$ be the subgroup of the Selmer group $\operatorname{Sel}_{l^{m}}(E / K)$ generated by $e_{i}$ for all $i \geq 2$. The element $\gamma_{M_{0}+N_{1}}(0)$ belongs to the component $l^{m} E(K)$ in the decomposition (5.4.1) of $\operatorname{Sel}_{l^{m}}(E / K)$. Hence, we have that the subgroup of $\operatorname{Sel}_{l^{m}}(E / K)$ generated by $S, e_{1}, \gamma_{M_{0}+N_{1}}(0)$ is the direct sum

$$
S \oplus \mathbb{Z} e_{1} \oplus \mathbb{Z} \gamma_{M_{0}+N_{1}}(0) .
$$

We may now apply Lemma 5.4.1 to $S$ and the elements $e_{1}, \gamma_{M_{0}+N_{1}}(0)$ where we take the parameters of the lemma to be

$$
c=0, \quad r=0, \quad s=M_{0}+N_{1}, \quad t=0, \quad n=N_{1} .
$$

There is according to the lemma a prime divisor $z_{1} \in \Lambda^{1}\left(M_{0}+N_{1}\right)$ which satisfies the following two conditions, where $y_{1} \in \Sigma_{K}$ is the prime divisor of $K$ lying over $z_{1}$, where the subscript $y_{1}$ denotes localization at $y_{1}$, and where the point $w_{1, y_{1}}$ in $\left(E\left(K_{y_{1}}\right) / l^{N_{1}} E\left(K_{y_{1}}\right)\right)^{\epsilon}$ is such that $\partial_{l^{N_{1}}}\left(w_{1, y_{1}}\right)=e_{1, y_{1}}$ :

$$
\operatorname{ord}\left[\delta_{M_{0}+N_{1}}\left(z_{1}\right)_{y_{1}}, w_{1, y_{1}}\right]_{y_{1}}=l^{N_{1}}
$$

and

$$
S_{y_{1}}=0 .
$$

Here in (5.4.6), $\delta_{M_{0}+N_{1}}\left(z_{1}\right)$ is the cohomology class in $H^{1}(K, E)_{l^{M_{0}+N_{1}}}$ associated to $z_{1}$.

Let

$$
\delta_{M_{0}}\left(z_{1}\right) \in \amalg(E / K)_{l^{\infty}}^{\epsilon}
$$

be the cohomology class associated to this prime divisor $z_{1} \in \Lambda^{1}\left(M_{0}+N_{1}\right)$; that $\delta_{M_{0}}\left(z_{1}\right)$ belongs to the Tate-Shafarevich group $\amalg(E / K)_{l_{\infty}}^{\epsilon}$ follows from Lemma 5.1.3(a).

On the one hand, $l^{N_{1}}$ is the maximum order of an element of $\amalg(E / K)_{l^{\infty}}^{\epsilon}$. From Proposition 5.2.1 and the isomorphism of (5.4.1), there is an element in the Selmer group $\operatorname{Sel}_{l \infty}(E / K)^{\epsilon}$, and hence in the Tate-Shafarevich group $\amalg(E / K)_{l \infty}^{\epsilon}$, of order $l^{M_{0}-M_{1}}$. It follows that we have the inequality

$$
M_{0}-M_{1} \leq N_{1} .
$$

On the other hand, the Cassels pairing gives, as $l^{n} \delta_{M_{0}}\left(z_{1}\right)=\delta_{M_{0}-n}\left(z_{1}\right)$ if $n \leq M_{0}$, that

$$
\begin{aligned}
\left\langle\delta_{M_{0}}\left(z_{1}\right), l^{n} d\right\rangle_{\text {Cassels }} & =\left\langle l^{n} \delta_{M_{0}}\left(z_{1}\right), d\right\rangle_{\text {Cassels }} \\
& =\left\langle\delta_{M_{0}-n}\left(z_{1}\right), d\right\rangle_{\text {Cassels }}
\end{aligned}
$$

where

$$
l^{N_{i}} \delta_{M_{0}+N_{i}-n}\left(z_{1}\right)=\delta_{M_{0}-n}\left(z_{1}\right) .
$$

By the construction of the Cassels pairing as a sum of local pairings, more precisely from Proposition 4.6.1 and equation (4.6.1), we obtain from (5.4.9) that 
for all $0 \leq n \leq N_{i}-1$ where $l^{N_{i}}$ is the order of $d_{i}$

$$
\begin{aligned}
\left\langle\delta_{M_{0}}\left(z_{1}\right), l^{n} d_{i}\right\rangle_{\text {Cassels }} & =\sum_{v \in \Sigma_{K}}\left[\delta_{M_{0}+N_{i}-n}\left(z_{1}\right)_{v}, w_{i, v}\right]_{v} \\
& =\left[\delta_{M_{0}+N_{i}-n}\left(z_{1}\right)_{y_{1}}, w_{i, y_{1}}\right]_{y_{1}}
\end{aligned}
$$

where $e_{i, v}=\partial_{l^{N_{i}}}\left(w_{i, v}\right)$ as in (5.4.3) for all $v \in \Sigma_{K}$, as we have that $\delta_{M_{0}+N_{i}-n}\left(z_{1}\right)_{v}=0$ for all places $v$ of $K$ not dividing $z_{1}$ (by Proposition 4.5.1(a)).

The term

$$
\left[\delta_{M_{0}+N_{i}-n}\left(z_{1}\right)_{y_{1}}, w_{i, y_{1}}\right]_{y_{1}}
$$

is zero if $i \geq 2$ by (5.4.7). Hence we have from (5.4.10) that

$$
\left\langle\delta_{M_{0}}\left(z_{1}\right), d_{i}\right\rangle_{\text {Cassels }}=0 \quad \text { for } i \geq 2 .
$$

Let $i=1$. From the sum formula (5.4.10) we have that

$$
\left\langle\delta_{M_{0}}\left(z_{1}\right), l^{n} d_{1}\right\rangle_{\text {Cassels }}=\left[\delta_{M_{0}+N_{1}-n}\left(z_{1}\right)_{y_{1}}, w_{1, y_{1}}\right]_{y_{1}} .
$$

By (5.4.6), the term $\left[\delta_{M_{0}+N_{i}}\left(z_{1}\right)_{y_{1}}, w_{1, y_{1}}\right]_{y_{1}}$ has order $l^{N_{1}}$. Hence by (5.4.12), the element $\left\langle\delta_{M_{0}}\left(z_{1}\right), d_{1}\right\rangle_{\text {Cassels }}$ of $\mathbb{Q} / \mathbb{Z}$ has order $l^{N_{1}}$. Hence, the character

$$
d \mapsto\left\langle\delta_{M_{0}}\left(z_{1}\right), l^{n} d\right\rangle_{\text {Cassels }}, \quad \amalg(E / K)_{l^{\infty}}^{\epsilon} \rightarrow \mathbb{Q} / \mathbb{Z},
$$

is nonzero for all $n$ such that $0 \leq n \leq N_{1}-1$ and more precisely

$$
\left\langle\delta_{M_{0}}\left(z_{1}\right), l^{n} d_{1}\right\rangle_{\text {Cassels }}
$$

is nonzero for all $n$ such that $0 \leq n \leq N_{1}-1$.

Therefore, the character

$$
d \mapsto\left\langle\delta_{M_{0}}\left(z_{1}\right), d\right\rangle_{\text {Cassels }}, \quad \amalg(E / K)_{l^{\infty}}^{\epsilon} \rightarrow \mathbb{Q} / \mathbb{Z},
$$

vanishes on $\prod_{i \geq 2} D_{i}$ (by (5.4.11)) and its restriction to $D_{1}$ generates the dual $\widehat{D}_{1}$. Hence, the element $\delta_{M_{0}}\left(z_{1}\right)$ of $\amalg(E / K)_{l^{\infty}}^{\epsilon}$ has order at least $l^{N_{1}}$, as this is the order of the cyclic group $D_{1}$. Since $\delta_{M_{0}}\left(z_{1}\right)$ has order at most $l^{M_{0}-M_{1}}$, by the definition of the cohomology class $\delta_{M_{0}}\left(z_{1}\right)$ (see Lemma 5.1.3(c)), we obtain that

$$
N_{1} \leq M_{0}-M_{1} \text {. }
$$

Hence we must have from the inequality (5.4.8) that

$$
N_{1}=M_{0}-M_{1} \text {. }
$$

It follows from this equality that $\delta_{M_{0}}\left(z_{1}\right)$ has order $l^{M_{0}-M_{1}}$. Therefore, $l^{M_{1}+1}$ does not divide $P_{z_{1}}$, and hence, we have that

$$
l^{M_{1}} \| P_{z_{1}} .
$$

In summary, we have shown that $\delta_{M_{0}}\left(z_{1}\right)$ has order $l^{N_{1}}=l^{M_{0}-M_{1}}, l^{M_{1}} \|$ $P_{z_{1}}, S_{y_{1}}=0$, the character $d \mapsto\left\langle\delta_{M_{0}}\left(z_{1}\right), d\right\rangle_{\text {Cassels }}$ vanishes on $\prod_{i \geq 2} D_{i}$, and its restriction to $D_{1}$ generates the dual $\widehat{D}_{1}$. 
We now proceed by induction. Suppose that there are prime divisors $z_{1}, \ldots, z_{k} \in \Lambda^{1}\left(M_{0}+N_{1}\right)$ such that

$$
e_{i, y_{j}}=0 \text { for all } i \neq j \text { and for all } j \text { such that } 1 \leq j \leq k
$$

and if

$$
c_{j}=z_{1}+\cdots+z_{j}
$$

then

$$
l^{M_{j}} \| P_{c_{j}}, \delta_{M_{j-1}}\left(c_{j}\right) \in \amalg(E / K)_{l^{\infty}}^{-\nu(j)}, \quad \text { for all } 1 \leq j \leq k,
$$

and the characters

$$
\chi_{j}: d \mapsto\left\langle\delta_{M_{j-1}}\left(c_{j}\right), d\right\rangle_{\text {Cassels }}, \quad 1 \leq j \leq k,
$$

vanish on $\prod_{i \geq k+1} D_{i}$ and form a triangular basis of the dual of $\prod_{i \leq k} D_{i}$ such that the restriction of $\chi_{j}$ to the cyclic subgroup $D_{j}$ is a basis for the dual $\widehat{D}_{j}$ for all $j=1, \ldots, k$. Suppose further, as we have shown, that

$$
M_{j-1}-M_{j}=N_{j}, \quad \text { for } 1 \leq j \leq k,
$$

and

$$
\operatorname{ord} \delta_{M_{j-1}}\left(c_{j}\right)=l^{N_{j}}, \quad \text { for } 1 \leq j \leq k .
$$

We have already proved the existence of the divisor $c_{1}=z_{1}$ and these properties of the previous paragraph of $e_{i, y_{1}}, P_{c_{1}}, \delta_{M_{0}}\left(c_{1}\right), \chi_{1}, N_{1}=M_{0}-M_{1}$, including (5.4.14), (5.4.15), (5.4.16), and (5.4.17) for $k=1$. Let $y_{i} \in \Sigma_{K}$ be the place of $K$ above $z_{i}$ for all $i=1, \ldots, k$.

Let $m$ be the integer already selected such that $l^{m} \geq \exp \left(\amalg(E / K)_{l}\right)$. The order of $\delta_{M_{k-1}}\left(c_{k}\right)$ in $\amalg(E / K)_{l^{\infty}}$ is the same as its order as a character on $D$ via the nondegenerate Cassels pairing. Since $D$ is an isotropic subgroup of $\amalg(E / K)_{l^{\infty}}$, it follows that

$$
\mathbb{Z} \delta_{M_{k-1}}\left(c_{k}\right) \cap D=0
$$

We have that

$$
\gamma_{M_{k}+N_{k+1}}\left(c_{k}\right)=l^{N_{k}-N_{k+1}} \gamma_{M_{k-1}}\left(c_{k}\right)
$$

as $N_{k}=M_{k-1}-M_{k}$ from (5.4.16) and where $N_{k}-N_{k+1} \geq 0$ by the definition of the integers $N_{i}$ as the invariants of $\amalg(E / K)_{l^{\infty}}$ in decreasing order. It follows from the induction hypothesis that the cohomology class $\delta_{M_{k}+N_{k+1}}\left(c_{k}\right)$ has order $l^{N_{k+1}}$, by (5.4.17), and belongs to $\amalg(E / K)_{l^{\infty}}^{-\nu(k)}$.

We have that $l^{M_{k}} \| P_{c_{k}}$ by the induction hypothesis (5.4.15). Hence by Proposition 3.4.1(a), the cohomology class $\gamma_{M_{k}+N_{k+1}}\left(c_{k}\right)$ then has the same order as its homomorphic image $\delta_{M_{k}+N_{k+1}}\left(c_{k}\right)$, namely, $l^{N_{k+1}}$.

Let $S$ be the subgroup of the Selmer group $\operatorname{Sel}_{l^{m}}(E / K)$ generated by the elements $e_{i}$ for all $i \neq k+1$. Let $T$ be the subgroup of the $\operatorname{Selmer}$ group $\operatorname{Sel}_{l^{m}}(E / K)$ generated by $\gamma_{M_{k}+N_{k+1}}\left(c_{k}\right), e_{k+1}, S$. From the isomorphism (5.4.1) and that $\mathbb{Z} \delta_{M_{k}+N_{k+1}}\left(c_{k}\right)$ has trivial intersection with $D$ by (5.4.18), there is an equality of 
subgroups of the Selmer group $\operatorname{Sel}_{l^{m}}(E / K)$, where the sums on the right-hand side are direct,

$$
T=\mathbb{Z} \gamma_{M_{k}+N_{k+1}}\left(c_{k}\right) \oplus \mathbb{Z} e_{k+1} \oplus S .
$$

We may now apply Lemma 5.4.1 to $S$ and the elements $e_{k+1}, \gamma_{M_{k}+N_{k+1}}\left(c_{k}\right)$ where we take

$$
c=c_{k}, \quad r=k, \quad s=M_{k}+N_{k+1}, \quad t=M_{0}+N_{1}-s, \quad n=N_{k+1} .
$$

There is then according to the lemma a prime divisor $z_{k+1} \in \Lambda^{1}\left(M_{0}+N_{1}\right)$ which satisfies the following two conditions:

$$
\operatorname{ord}\left[\delta_{M_{k}+N_{k+1}}\left(c_{k}+z_{k+1}\right), w_{k+1, y_{k+1}}\right]_{y_{k+1}}=l^{N_{k+1}}
$$

and

$$
S_{y_{k+1}}=0,
$$

where $y_{k+1} \in \Sigma_{K}$ is the prime divisor of $K$ lying over $z_{k+1}$, the subscript $y_{k+1}$ denotes localization at $y_{k+1}$, and the point $w_{k+1, y_{k+1}}$ in $\left(E\left(K_{y_{k+1}}\right) /\right.$ $\left.l^{N_{k+1}} E\left(K_{y_{k+1}}\right)\right)^{\epsilon}$ is such that $\partial_{l^{N_{k+1}}}\left(w_{k+1, y_{k+1}}\right)=e_{k+1, y_{k+1}}$.

For this selection of $z_{k+1} \in \Lambda^{1}\left(M_{0}+N_{1}\right)$, note that $M_{k}+N_{k+1} \leq M_{0}+N_{1}$ and so $t \geq 0$, where $t$ is the parameter of (5.4.19), because $M_{r}, N_{r}$ are both decreasing sequences of integers, and note that $H^{1}\left(K, E_{l^{M_{k}+N_{k+1}}}\right)$ is a subgroup of $H^{1}\left(K, E_{l^{M_{0}+N_{1}}}\right)$ by Lemma 3.1.4.

Let $c_{k+1}$ be the divisor which is the sum of the $z_{i}$, for $i=1, \ldots, k+1$,

$$
c_{k+1}=\sum_{j=1}^{k+1} z_{j} .
$$

Let

$$
\delta_{M_{k}}\left(c_{k+1}\right) \in \amalg(E / K)_{l^{\infty}}^{-\nu(k+1)}
$$

be the cohomology class associated to this divisor $c_{k+1} \in \Lambda^{k+1}\left(M_{0}+N_{1}\right)$; that $\delta_{M_{k}}\left(c_{k+1}\right)$ belongs to the Tate-Shafarevich group $\amalg(E / K)_{l^{\infty}}^{-\nu(k+1)}$ follows from Lemma 5.1.3(a).

Then for $0 \leq n \leq N_{k+1}-1$ by the construction of the Cassels pairing as a sum of local terms, more precisely from Proposition 4.6.1 and (4.6.1), we have the following sum formulae as $d_{i}$ has order $l^{N_{i}}$ :

$$
\begin{aligned}
\left\langle\delta_{M_{k}}\left(c_{k+1}\right), l^{n} d_{i}\right\rangle_{\text {Cassels }} & =\left\langle\delta_{M_{k}-n}\left(c_{k+1}\right), d_{i}\right\rangle_{\text {Cassels }} \\
& =\sum_{v \in \Sigma_{K}}\left[\delta_{M_{k}-n+N_{i}}\left(c_{k+1}\right)_{v}, w_{i, v}\right]_{v} \\
& =\sum_{j=1}^{k+1}\left[\delta_{M_{k}-n+N_{i}}\left(c_{k+1}\right)_{y_{j}}, w_{i, y_{j}}\right]_{y_{j}},
\end{aligned}
$$

because $\delta_{M_{k}-n+N_{i}}\left(c_{k+1}\right)_{v}=0$ for all $v \in \Sigma_{K}$ not dividing an element of $\operatorname{Supp}\left(c_{k+1}\right)$ by Proposition 4.5.1(a). Here $w_{i, y_{j}} \in{ }_{l^{N_{i}}} E\left(K_{y_{j}}\right)$ is an element already chosen (see 
(5.4.3)) such that

$$
\partial_{l^{N_{i}}}\left(w_{i, y_{j}}\right)=e_{i, y_{j}}
$$

We have the following sum for the Cassels pairing, obtained from those of (5.4.23):

$$
\left\langle\delta_{M_{k}}\left(c_{k+1}\right), l^{n} d_{i}\right\rangle_{\text {Cassels }}=\sum_{j=1}^{k+1}\left[\delta_{M_{k}-n+N_{i}}\left(c_{k+1}\right)_{y_{j}}, w_{i, y_{j}}\right]_{y_{j}} .
$$

We have $w_{i, y_{j}}=0$ for all $i \neq j$ and all $j$ such that $1 \leq j \leq k+1$ by (5.4.14) for $j \leq k$ and by (5.4.21) for $j=k+1$. It follows that for $i \geq k+1$ all terms $\left[\delta_{M_{k}-n+N_{i}}\left(c_{k+1}\right)_{y_{j}}, w_{i, y_{j}}\right]_{y_{j}}$ of this sum (5.4.24) are zero except the last $\left[\delta_{M_{k}-n+N_{i}}\left(c_{k+1}\right)_{y_{k+1}}, w_{i, y_{k+1}}\right]_{y_{k+1}}$ and the entire sum is zero for $i>k+1$.

By (5.4.20) the local term

$$
\left[\delta_{M_{k}+N_{k+1}-n}\left(c_{k+1}\right)_{y_{k+1}}, w_{k+1, y_{k+1}}\right]_{y_{k+1}}
$$

is nonzero for all integers $n$ such that $0 \leq n \leq N_{k+1}-1$.

Therefore the character, by $(5.4 .24)$,

$$
\chi_{k+1}: d \mapsto\left\langle\delta_{M_{k}}\left(c_{k+1}\right), d\right\rangle_{\text {Cassels }}
$$

vanishes on $\prod_{i>k+1} D_{i}$ and its restriction to $D_{k+1}$ generates $\widehat{D}_{k+1}$, as $D_{k+1}$ has order $l^{N_{k+1}}$ by definition. Hence, $\chi_{k+1}$ extends the triangular basis $\chi_{1}, \ldots, \chi_{k}$ to generate $\prod_{i \leq k+1} \widehat{D}_{i}$ and $\delta_{M_{k}}\left(c_{k+1}\right)$ has order at least $l^{N_{k+1}}$. Since it has order at most $l^{M_{k}-M_{k+1}}$, by the definition of the cohomology class $\delta_{M_{k}}\left(c_{k+1}\right)$ (see Lemma 5.1.3(c)), we conclude that

$$
N_{k+1} \leq M_{k}-M_{k+1}
$$

and also

$$
l^{N_{k+1}} \leq \operatorname{ord} \delta_{M_{k}}\left(c_{k+1}\right) \leq l^{M_{k}-M_{k+1}} .
$$

Let $C_{k}$ be the subgroup of the Selmer eigencomponent $\operatorname{Sel}_{l^{m}}(E / K)^{-\nu(k+1)}$ given by

$$
C_{k}=\left(\gamma_{M_{0}+N_{1}}(0), e_{1}, \ldots, e_{k}, \gamma_{M_{0}}\left(c_{1}\right), \ldots, \gamma_{M_{k-1}}\left(c_{k}\right)\right)^{-\nu(k+1)} .
$$

If $k$ is even, then $C_{k}$ is generated by $e_{1}, \gamma_{M_{0}}\left(c_{1}\right), e_{3}, \gamma_{M_{2}}\left(c_{3}\right), \ldots, e_{k-1}, \gamma_{M_{k-2}}\left(c_{k-1}\right)$ of which there are $k$ in number. If $k$ is odd, then $C_{k}$ is generated by $\gamma_{M_{0}+N_{1}}(0), e_{2}, \gamma_{M_{1}}\left(c_{2}\right), e_{4}, \gamma_{M_{3}}\left(c_{4}\right), \ldots, e_{k-1}, \gamma_{M_{k-2}}\left(c_{k-1}\right)$ of which there are $k$ in number. We then have for all integers $k$ that

$$
\operatorname{rank}\left(C_{k}\right) \leq k .
$$

The elements $e_{1}, \ldots, e_{k}$ generate a subgroup of $\operatorname{Sel}_{l^{m}}(E / K)$ isomorphic to $\prod_{i \leq k} D_{i}$ by the decomposition (5.4.1) of the Selmer group. Furthermore, the elements $\gamma_{M_{0}}\left(c_{1}\right), \ldots, \gamma_{M_{k-1}}\left(c_{k}\right)$ generate a subgroup of $\operatorname{Sel}_{l^{m}}(E / K)$ isomorphic to the dual of $\prod_{i<k} D_{i}$ because $\gamma_{M_{i-1}}\left(c_{i}\right)$ has the same order as $\delta_{M_{i-1}}\left(c_{i}\right)$ for all $i=1, \ldots, k$ (see (5.4.15), (5.4.16), (5.4.17), and Proposition 3.4.1(a)). In the 
decomposition (5.4.1) of $\operatorname{Sel}_{l^{m}}(E / K)$, take $S$ to be the subgroup $l^{m} E(K) \oplus_{1 \leq i \leq k}$ $\prod D_{i} \oplus \bigoplus_{1 \leq i \leq k} \mathbb{Z} \gamma_{M_{i-1}}\left(c_{i}\right)$. Then we have $C_{k}=S^{-\nu(k+1)}$.

If a finite abelian group $G$ is a direct product $G_{1} \times G_{2}$ of two subgroups and $g \in G$ is such that $\mathbb{Z} g \cap G_{1}=0$, then the order of the element $g$ is at most the exponent of $G_{2}$. Then by the previous remark where we take $G=$ $\operatorname{Sel}_{l^{m}}(E / K)^{-\nu(k+1)}$ and $G_{1}=C_{k}$, we have that $l^{N_{k+1}}$ is the maximum order of an element $c \in \operatorname{Sel}_{l^{m}}(E / K)^{-\nu(k+1)}$ if

$$
\mathbb{Z} c \cap C_{k}=0
$$

by the decomposition (5.4.1) of the Selmer group $\operatorname{Sel}_{l^{m}}(E / K)$.

On the other hand, by Proposition 5.2.1 and (5.4.27) applied to subgroup $C_{k}$ of the Selmer group there is an element in $\operatorname{Sel}_{l^{m}}(E / K)^{-\nu(k+1)}$ of order $l^{M_{k}-M_{k+1}}$ satisfying $\mathbb{Z} c \cap C_{k}=0$. Hence, we have that

$$
M_{k}-M_{k+1} \leq N_{k+1}
$$

and so by (5.4.25) we have that

$$
N_{k+1}=M_{k}-M_{k+1} .
$$

It follows from this equality and (5.4.26) that

$$
\operatorname{ord} \delta_{M_{k}}\left(c_{k+1}\right)=l^{M_{k}-M_{k+1}}
$$

Therefore, $l^{M_{k+1}+1}$ does not divide $P_{c_{k+1}}$ and $l^{M_{k+1}} \mid P_{c_{k+1}}$, and hence we have

$$
l^{M_{k+1}} \| P_{c_{k+1}} \text {. }
$$

The properties (5.4.28), (5.4.29), and (5.4.30) complete the proof of the induction step and this proves Theorems 4.1.4 and 4.1.8.

\subsection{Proofs of Theorems 1.1 .1 and 4.1 .9}

Proof of Theorem 1.1.1

From Proposition 2.2.1, we have, because $l \in \mathcal{P}$ is an odd prime number, that $\amalg(E / F)_{l \infty} \cong \amalg(E / K)_{l \infty}^{+1}$. The theorem now follows immediately from Theorem 4.1.4.

Proof of Theorem 4.1.9

As $P_{0}$ has infinite order, by Lemma 5.1.1 and [1, Theorem 7.6.5] we have that $M_{0}$ is finite, the group $\mathbb{Z} P_{0}$ has finite index in $E(K)$, and the highest power of $l$ dividing the index $\left[E(K): \mathbb{Z} P_{0}\right]$ is equal to

$$
l^{M_{0}}=\left|\left(E(K) / \mathbb{Z} P_{0}\right)_{l^{\infty}}\right| .
$$

By Theorem 4.1.4, we have that

$$
\left|\amalg(E / K)_{l}\right|=\prod_{i \geq 0} l^{2\left(M_{i}-M_{i+1}\right)}=l^{2\left(M_{0}-M_{\infty}\right)},
$$

where

$$
M_{\infty}=\min _{i \in \mathbf{N}} M_{i}
$$


and where this minimum exists because the $M_{i}$ 's form a decreasing sequence of nonnegative integers by Lemma 5.1.2.

\subsection{Generators of Tate-Shafarevich groups}

\subsection{1.}

The notation from Section 5.1.1 also holds for this section. We further denote by

$\Sigma_{K}$ the set of all places of the global field $K$;

$[\cdot, \cdot]_{v}: l^{m} E\left(K_{v}\right) \times H^{1}\left(K_{v}, E\right)_{l^{m}} \rightarrow \mathbb{Z} / l^{m} \mathbb{Z}$ the nondegenerate local pairing at the place $v$ of $K$ induced by the cup product, as in Theorem 2.1.2.

\section{THEOREM 5.6.1}

Let $l$ be a prime number belonging to $\mathcal{P}$; assume that $l$ is coprime to the order of $\operatorname{Pic}(A)$. Suppose that $P_{0}$ has infinite order in $E(K)$, and let a be an integer such that $a \geq 2 M_{0}$. Then we have that

(a) the classes $\delta_{M_{0}}(c)$, for all $c \in \Lambda^{1}(a)$, generate $\amalg(E / K)_{l_{\infty}}^{\epsilon}$; and

(b) the classes $\delta_{M_{1}}(c)$, for all $c \in \Lambda^{2}(a)$, generate $\amalg(E / K)_{l \infty}^{-\epsilon}$.

\section{THEOREM 5.6.2}

Under the hypotheses of Theorem 5.6.1, the classes $\delta_{M_{r}}(c)$, for all $c \in \Lambda^{r}(a)$, generate $\amalg(E / F)_{l^{\infty}}$ where $r=(3-\epsilon) / 2$.

Proof

This obviously follows from Theorem 5.6.1 and Proposition 2.2.1.

Proof of Theorem 5.6.1

By Theorem 4.1.4 above or [1, Theorem 7.6.5], the group $\amalg(E / K)_{l_{\infty}}$ is finite. By Lemmas 5.1.1 and 5.1.2, the quantities $M_{0}, M_{1}, M_{2}, \ldots$ are all finite and form a decreasing sequence of nonnegative integers. We fix an integer $a \geq 2 M_{0}$. The classes $\delta_{M_{0}}(z)$, for $z \in \Lambda^{1}(a)$, and the classes $\delta_{M_{1}}\left(z_{1}+z_{2}\right)$, for $z_{1}+z_{2} \in \Lambda^{2}(a)$, belong to $\amalg(E / K)_{l}$ by Lemma 5.1.3(a). We now prove separately the two parts of the theorem.

(a) Suppose that $d \in \amalg(E / K)_{l^{\infty}}^{\epsilon}$ has order exactly $l^{M}$ for some $M>0$ and is in the $\epsilon$-eigenspace of $\amalg(E / K)_{l \infty}$. By Theorem 4.1.10 there is an isomorphism of $\epsilon$-components

$$
\operatorname{Sel}_{l^{\infty}}(E / K)^{\epsilon} \cong \amalg(E / K)_{l^{\infty}}^{\epsilon}
$$

induced from the natural surjection of the Selmer group onto the Tate-Shafarevich group.

By Theorem 4.1.4, we have $M \leq M_{0}$. By the isomorphism (5.6.1), we may lift $d$ to an element of the Selmer group $e \in \operatorname{Sel}_{l}(E / K)^{\epsilon}$ of order $l^{M}$. The cohomology class $\gamma_{M_{0}+M}(0)$ belongs to the $-\epsilon$-eigenspace $\operatorname{Sel}_{l \infty}(E / K)^{-\epsilon}$ of the Selmer group and has order $l^{M}$ by Lemma 5.1.1. 
We may apply Lemma 5.4.1 to the elements $e, \gamma_{M_{0}+M}(0)$ and the subgroup $S=0$ where we take the parameters of the lemma to be

$$
c=0, \quad r=0, \quad s=M_{0}+M, \quad t=a-\left(M_{0}+M\right), \quad n=M .
$$

Note that $t \geq 0$ as $a \geq 2 M_{0} \geq M_{0}+M$. There is according to the lemma a prime divisor $z \in \Lambda^{1}(a)$ which satisfies the following condition:

$$
\operatorname{ord}\left[\delta_{M_{0}+M}(z)_{y}, w_{y}\right]_{y}=l^{M},
$$

where $y \in \Sigma_{K}$ is the prime divisor of $K$ lying over $z$, where the subscript $y$ denotes localization at $y$, and where the point $w_{y}$ in $\left(E\left(K_{y}\right) / l^{M} E\left(K_{y}\right)\right)^{\epsilon}$ is such that $\partial_{l^{M}}\left(w_{y}\right)=e_{y}$. Here in (5.6.3), $\delta_{M_{0}+M}(z)$ is the cohomology class in $H^{1}(K, E)_{l^{M_{0}+M}}$ associated to $z$.

Let $\delta_{M_{0}}(z)$ be the cohomology class of $\amalg(E / K)_{l^{\infty}}^{\epsilon}$ associated to $z$, where this class belongs to the Tate-Shafarevich group by Lemma 5.1.3.

The Cassels pairing gives, as $l^{u} \delta_{M_{0}}(z)=\delta_{M_{0}-u}(z)$ if $u \leq M_{0}$,

$$
\left\langle\delta_{M_{0}}(z), l^{u} d\right\rangle_{\text {Cassels }}=\left\langle\delta_{M_{0}-u}(z), d\right\rangle_{\text {Cassels }} .
$$

Because $d$ has order $l^{M}$ and that we have

$$
l^{M} \delta_{M_{0}+M-u}(z)=\delta_{M_{0}-u}(z),
$$

by the construction of the Cassels pairing in terms of local Tate pairings (see Proposition 4.6.1 and (4.6.1)) we obtain from (5.6.4)

$$
\left\langle\delta_{M_{0}}(z), l^{u} d\right\rangle_{\text {Cassels }}=\left[\delta_{M_{0}+M-u}(z)_{y}, w_{y}\right]_{y}
$$

where as above

$$
e_{y}=\partial_{l^{M}}\left(w_{y}\right),
$$

and where $w_{y} \in_{l^{M}} E\left(K_{y}\right)$ as we have that $\delta_{M_{0}+M-u}(z)_{v}=0$ for all places $v$ of $K$ not dividing $z$ by Proposition 4.5.1(a).

By (5.6.3) the element given by the Tate pairing

$$
\left[\delta_{M_{0}+M-u}(z)_{y}, w_{y}\right]_{y}
$$

of $\mathbb{Q} / \mathbb{Z}$ is nonzero for all integers $u$ such that $1 \leq u \leq M-1$ and hence

$$
\left\langle\delta_{M_{0}}(z), l^{u} d\right\rangle_{\text {Cassels }}
$$

is nonzero for all integers $u$ such that $1 \leq u \leq M-1$. We obtain that the character, where $z \in \Lambda^{1}(a)$,

$$
f \mapsto\left\langle\delta_{M_{0}}(z), f\right\rangle_{\text {Cassels }}, \quad \amalg(E / K)_{l \infty} \rightarrow \mathbb{Q} / \mathbb{Z}
$$

of $\amalg(E / K)_{l \infty}$ when restricted to $\mathbb{Z} d$ generates the dual $\widehat{\mathbb{Z} d}$ of this subgroup $\mathbb{Z} d$.

As $d$ is any element of the abelian group $\amalg(E / K)_{l^{\infty}}$, the nondegeneracy of Cassels pairing implies that the classes $\left\{\delta_{M_{0}}(z), z \in \Lambda^{1}(a)\right\}$ generate the $\epsilon$ eigenspace $\amalg(E / K)_{l^{\infty}}^{\epsilon}$, which proves the part of the theorem for $\amalg(E / K)_{l^{\infty}}^{\epsilon}$.

(b) Suppose that $f \in \amalg(E / K)_{l^{\infty}}^{-\epsilon}$ has order exactly $l^{M^{\prime}}$ and is in the $-\epsilon$ eigenspace of $\amalg(E / K)_{l^{\infty}}$. We have $M^{\prime} \leq M_{0}$ by Theorem 4.1.4. 
We have from Theorem 4.1.10 an isomorphism compatible with the $\tau$ eigenspaces

$$
\operatorname{Sel}_{l^{m}}(E / K) \cong l_{l^{m}} E(K) \oplus \amalg(E / K)_{l^{\infty}} \quad \text { for all } m \geq N_{1}
$$

from which as stated in this theorem we obtain the isomorphism, taking $m=a \geq$ $2 M_{0} \geq N_{1}$,

$$
\Delta_{a}: \operatorname{Sel}_{l^{a}}(E / K)^{-\epsilon} \cong \mathbb{Z} / l^{a} \mathbb{Z} \oplus \amalg(E / K)_{l^{\infty}}^{-\epsilon} .
$$

The projection of $\operatorname{Sel}_{l^{a}}(E / K)$ onto the second factor $\amalg(E / K)_{l^{\infty}}$ of this direct sum is the natural surjection of the Selmer group onto the Tate-Shafarevich group.

Lift $f$ to $g \in \operatorname{Sel}_{l^{a}}(E / K)^{-\epsilon}$ via this isomorphism $\Delta_{a}$ for the integer $a$ where $g$ has order $l^{M^{\prime}}$ and has zero component in the first term $\mathbb{Z} / l^{a} \mathbb{Z}$ of this decomposition of the Selmer group. By Theorem 4.1.4 or alternatively Proposition 5.2.1, there is an element $d \in \amalg(E / K)_{l^{\infty}}^{\epsilon}$ of order exactly $l^{M_{0}-M_{1}}$. Via the isomorphism (5.6.1), lift $d$ to an element of the Selmer group $e \in \operatorname{Sel}_{l^{a}}(E / K)^{\epsilon}$ which is also of order $l^{M_{0}-M_{1}}$. The cohomology class $\gamma_{2 M_{0}-M_{1}}(0)$ belongs to the $-\epsilon$-eigenspace $\operatorname{Sel}_{l^{a}}(E / K)^{-\epsilon}$ of the Selmer group and has order $l^{M_{0}-M_{1}}$ by Lemma 5.1.1.

Let $T$ be the subgroup of $\operatorname{Sel}_{l^{a}}(E / K)$ generated by the three elements $\gamma_{2 M_{0}-M_{1}}(0), e$, and $g$. As the two elements $\gamma_{2 M_{0}-M_{1}}(0)$ and $g$ belong to the different components of $\operatorname{Sel}_{l^{a}}(E / K)^{-\epsilon}$ under the isomorphism $\Delta_{a}$ and as $e$ belongs to a different eigenspace $\operatorname{Sel}_{l^{a}}(E / K)^{\epsilon}$, the group $T$ is the direct sum of the subgroups generated by these three elements; that is to say, we have that

$$
T \cong \mathbb{Z} \gamma_{2 M_{0}-M_{1}}(0) \oplus \mathbb{Z} e \oplus \mathbb{Z} g
$$

We may apply Lemma 5.4.1 to the subgroup $S=\mathbb{Z} g$ and the elements $\gamma_{2 M_{0}-M_{1}}(0)$ and $e$ of the Selmer group; we take the parameters of the lemma to be

$$
\begin{aligned}
& c=0, \quad r=0, \quad s=2 M_{0}-M_{1}, \\
& t=a-\left(2 M_{0}-M_{1}\right), \quad n=M_{0}-M_{1} .
\end{aligned}
$$

Note that $t \geq 0$ as $a \geq 2 M_{0}$. There is according to the lemma a prime divisor $z_{1} \in \Lambda^{1}(a)$ which satisfies the following two conditions:

$$
\begin{aligned}
\operatorname{ord}\left[\delta_{2 M_{0}-M_{1}}\left(z_{1}\right)_{y_{1}}, w_{y_{1}}\right]_{y_{1}} & =l^{M_{0}-M_{1}}, \\
(\mathbb{Z} g)_{y_{1}} & =0,
\end{aligned}
$$

where $y_{1} \in \Sigma_{K}$ is the prime divisor of $K$ lying over $z_{1}$ and where the point $w_{y_{1}}$ in $\left(E\left(K_{y_{1}}\right) / l^{M_{0}-M_{1}} E\left(K_{y_{1}}\right)\right)^{\epsilon}$ is such that $\partial_{l^{M_{0}-M_{1}}}\left(w_{y_{1}}\right)=e_{y_{1}}$. Here in (5.6.6), $\delta_{2 M_{0}-M_{1}}\left(z_{1}\right)$ is the cohomology class in $H^{1}(K, E)_{l^{2 M_{0}-M_{1}}}$ associated to $z_{1}$.

Let

$$
\delta_{M_{0}}\left(z_{1}\right) \in \amalg(E / K)_{l^{\infty}}^{\epsilon}
$$

be the cohomology class associated to this prime divisor $z_{1} \in \Lambda^{1}(a)$; that $\delta_{M_{0}}\left(z_{1}\right)$ belongs to $\amalg(E / K)_{l \infty}^{\epsilon}$ follows from Lemma 5.1.3(a). 
The Cassels pairing gives, as $l^{u} \delta_{M_{0}}\left(z_{1}\right)=\delta_{M_{0}-u}\left(z_{1}\right)$ if $u \leq M_{0}$,

$$
\begin{aligned}
\left\langle\delta_{M_{0}}\left(z_{1}\right), l^{u} d\right\rangle_{\text {Cassels }} & =\left\langle l^{u} \delta_{M_{0}}\left(z_{1}\right), d\right\rangle_{\text {Cassels }} \\
& =\left\langle\delta_{M_{0}-u}\left(z_{1}\right), d\right\rangle_{\text {Cassels }},
\end{aligned}
$$

where

$$
l^{M_{0}-M_{1}} \delta_{2 M_{0}-M_{1}-u}\left(z_{1}\right)=\delta_{M_{0}-u}\left(z_{1}\right) .
$$

By the construction of the Cassels pairing as a sum of local terms, more precisely from Proposition 4.6.1 and (4.6.1), we obtain from (5.6.8) that for all $0 \leq u \leq M_{0}-M_{1}-1$ where $M_{0}-M_{1}$ is the order of $d$

$$
\begin{aligned}
\left\langle\delta_{M_{0}}\left(z_{1}\right), l^{u} d\right\rangle_{\text {Cassels }} & =\sum_{v \in \Sigma_{K}}\left[\delta_{2 M_{0}-M_{1}-u}\left(z_{1}\right)_{v}, w_{v}\right]_{v} \\
& =\left[\delta_{2 M_{0}-M_{1}-u}\left(z_{1}\right)_{y_{1}}, w_{y_{1}}\right]_{y_{1}},
\end{aligned}
$$

where $e_{v}=\partial_{l^{M_{0}-M_{1}}}\left(w_{v}\right)$ for all $v \in \Sigma_{K}$, and where we have that $\delta_{2 M_{0}-M_{1}-u}\left(z_{1}\right)_{v}=$ 0 for all places $v$ of $K$ not dividing $z_{1}$ (by Proposition 4.5.1(a)).

From the sum formula (5.6.9) we have that

$$
\left\langle\delta_{M_{0}}\left(z_{1}\right), l^{u} d\right\rangle_{\text {Cassels }}=\left[\delta_{2 M_{0}-M_{1}-u}\left(z_{1}\right)_{y_{1}}, w_{y_{1}}\right]_{y_{1}} .
$$

By (5.6.6), $\left[\delta_{2 M_{0}-M_{1}}\left(z_{1}\right)_{y_{1}}, w_{y_{1}}\right]_{y_{1}}$ has order $l^{M_{0}-M_{1}}$.

Hence, the map

$$
s \mapsto\left\langle\delta_{M_{0}}\left(z_{1}\right), l^{u} s\right\rangle_{\text {Cassels }}, \quad \amalg(E / K)_{l^{\infty}}^{\epsilon} \rightarrow \mathbb{Q} / \mathbb{Z}
$$

is nonzero for all $u$ such that $0 \leq u \leq M_{0}-M_{1}-1$ and more precisely

$$
\left\langle\delta_{M_{0}}\left(z_{1}\right), l^{u} d\right\rangle_{\text {Cassels }}
$$

is nonzero for all $u$ such that $0 \leq u \leq M_{0}-M_{1}-1$.

Therefore, the character

$$
s \mapsto\left\langle\delta_{M_{0}}\left(z_{1}\right), s\right\rangle_{\text {Cassels }}, \quad \amalg(E / K)_{l \infty}^{\epsilon} \rightarrow \mathbb{Q} / \mathbb{Z}
$$

is such that its restriction to $\mathbb{Z} d$ generates the dual $\widehat{\mathbb{Z} d}$. Hence, $\delta_{M_{0}}\left(z_{1}\right)$ has order at least $l^{M_{0}-M_{1}}$ as this is the order of the cyclic group $\mathbb{Z} d$. Since $\delta_{M_{0}}\left(z_{1}\right)$ has order at most $l^{M_{0}-M_{1}}$, by the definition of the cohomology class $\delta_{M_{0}}\left(z_{1}\right)$ (see Lemma 5.1.3(c)), we obtain that $\delta_{M_{0}}\left(z_{1}\right)$ has order given by

$$
\operatorname{ord} \delta_{M_{0}}\left(z_{1}\right)=l^{M_{0}-M_{1}} \text {. }
$$

We have $l^{M_{1}} \mid P_{z_{1}}$ by the definition of $M_{1}$. It follows that $\gamma_{M_{0}}\left(z_{1}\right)$ has order less than or equal to $l^{M_{0}-M_{1}}$ by Proposition 3.4.1(a). As $\delta_{M_{0}}\left(z_{1}\right)$ has order $l^{M_{0}-M_{1}}$ by the previous paragraph and $\delta_{M_{0}}\left(z_{1}\right)$ is a homomorphic image of $\gamma_{M_{0}}\left(z_{1}\right)$ we must have that $\gamma_{M_{0}}\left(z_{1}\right)$ has order exactly given by

$$
\operatorname{ord} \gamma_{M_{0}}\left(z_{1}\right)=l^{M_{0}-M_{1}} \text {. }
$$

Therefore, we have by Proposition 3.4.1(a) that

$$
l^{M_{1}} \| P_{z_{1}} .
$$


We may apply Lemma 5.4.1 to the subgroup $S=0$ and the two elements $\gamma_{M_{1}+M^{\prime}}\left(z_{1}\right)$ and $g$ of the Selmer group which both have order $l^{M^{\prime}}$; we take the parameters of the lemma to be

$$
\begin{aligned}
& c=z_{1}, \quad r=1, \quad s=M_{1}+M^{\prime}, \\
& t=a-\left(M_{1}+M^{\prime}\right), \quad n=M^{\prime} .
\end{aligned}
$$

Note that $t \geq 0$ as $a \geq 2 M_{0}$ and $M^{\prime}, M_{1} \leq M_{0}$. There is according to the lemma a prime divisor $z_{2} \in \Lambda^{1}(a)$ which satisfies the following condition:

$$
\operatorname{ord}\left[\delta_{M_{1}+M^{\prime}}\left(z_{1}+z_{2}\right)_{y_{2}}, x_{y_{2}}\right]_{y_{2}}=l^{M^{\prime}},
$$

where $y_{2} \in \Sigma_{K}$ is the prime divisor of $K$ lying over $z_{2}$ and where the point $x_{y_{2}}$ in $\left(E\left(K_{y_{2}}\right) / l^{M^{\prime}} E\left(K_{y_{2}}\right)\right)^{\epsilon}$ is such that $\partial_{l^{M^{\prime}}}\left(x_{y_{2}}\right)=e_{y_{2}}$. Here in (5.6.11), $\delta_{M_{1}+M^{\prime}}\left(z_{1}+z_{2}\right)$ is the cohomology class in $H^{1}(K, E)_{l^{M_{1}+M^{\prime}}}$ associated to $z_{1}+z_{2}$.

Let

$$
\delta_{M_{1}}\left(z_{1}+z_{2}\right)
$$

be the cohomology class of $\amalg(E / K)_{l^{\infty}}^{-\epsilon}$ associated to the divisor $z_{1}+z_{2} \in \Lambda^{2}(a)$, where this class belongs to the Tate-Shafarevich group by Lemma 5.1.3(a).

Then the Cassels pairing gives, as $l^{u} \delta_{M_{1}}\left(z_{1}+z_{2}\right)=\delta_{M_{1}-u}\left(z_{1}+z_{2}\right)$ if $u \leq M_{1}$,

$$
\left\langle\delta_{M_{1}}\left(z_{1}+z_{2}\right), l^{u} f\right\rangle_{\text {Cassels }}=\left\langle\delta_{M_{1}-u}\left(z_{1}+z_{2}\right), f\right\rangle_{\text {Cassels }} .
$$

Because $f$ has order $l^{M^{\prime}}$ and that we have

$$
l^{M^{\prime}} \delta_{M_{1}+M^{\prime}-u}\left(z_{1}+z_{2}\right)=\delta_{M_{1}-u}\left(z_{1}+z_{2}\right),
$$

by the construction of the Cassels pairing (see Proposition 4.6.1 and (4.6.1)) we obtain from (5.6.12) that

$$
\left\langle\delta_{M_{1}}\left(z_{1}+z_{2}\right), l^{u} f\right\rangle_{\text {Cassels }}=\sum_{z \in \operatorname{Supp}\left(z_{1}+z_{2}\right)}\left[\delta_{M_{1}+M^{\prime}-u}\left(z_{1}+z_{2}\right)_{y}, x_{y}\right]_{y},
$$

where

$$
g_{y}=\partial_{l^{M^{\prime}}}\left(x_{y}\right)
$$

and

$$
x_{y} \in\left({ }_{l^{M^{\prime}}} E\left(K_{y}\right)\right)^{-\epsilon}
$$

because we have that $\delta_{M_{1}+M-u}\left(z_{1}+z_{2}\right)_{v}=0$ for all places $v$ of $K$ not dividing $z_{1}+z_{2}$ by Proposition 4.5.1(a). Here in (5.6.13) and (5.6.14), $z$ runs over the places $z_{1}, z_{2}$ and $y \in \Sigma_{K}$ runs over the place of $K$ above $z$, that is to say, above $z_{1}, z_{2}$.

The first term $\left[\delta_{M_{1}+M^{\prime}-u}\left(z_{1}+z_{2}\right)_{y_{1}}, x_{y_{1}}\right]_{y_{1}}$ in the sum (5.6.13) is zero by (5.6.7) and (5.6.14).

By (5.6.11), the second term, an element of $\mathbb{Q} / \mathbb{Z}$,

$$
\left[\delta_{M_{1}+M^{\prime}-u}\left(z_{1}+z_{2}\right)_{y_{2}}, x_{y_{2}}\right]_{y_{2}}
$$


is nonzero for $0 \leq u \leq M^{\prime}-1$ and hence $\delta_{M_{1}}\left(z_{1}+z_{2}\right)$ has order at least $l^{M^{\prime}}$. We obtain from this and (5.6.13) that the Cassels pairing

$$
\left\langle\delta_{M_{1}}\left(z_{1}+z_{2}\right), l^{u} f\right\rangle_{\text {Cassels }}=\left[\delta_{M_{1}+M^{\prime}-u}\left(z_{1}+z_{2}\right)_{y_{1}}, x_{y_{1}}\right]_{y_{1}}
$$

is nonzero for all $u$ such that $0 \leq u \leq M^{\prime}-1$. Hence, this character

$$
s \mapsto\left\langle\delta_{M_{1}}\left(z_{1}+z_{2}\right), s\right\rangle_{\text {Cassels }}, \quad \amalg(E / K)_{l^{\infty}}^{-\epsilon} \rightarrow \mathbb{Q} / \mathbb{Z}
$$

has restriction to $\mathbb{Z} f$, which generates the dual of this subgroup $\mathbb{Z} f$ of order $l^{M^{\prime}}$.

As $f$ is any element of the abelian group $\amalg(E / K)_{l \infty}^{-\epsilon}$, the nondegeneracy of the Cassels pairing on $\amalg(E / K)_{l^{\infty}}^{-\epsilon}$ implies that the classes $\delta_{M_{1}}\left(z_{1}+z_{2}\right)$, for divisors $z_{1}+z_{2} \in \Lambda^{2}(a)$, generate the $-\epsilon$-eigenspace $\amalg(E / K)_{l^{\infty}}^{-\epsilon}$, which proves the part of the theorem for $\amalg(E / K)_{l^{\infty}}^{-\epsilon}$.

\section{References}

[1] M. L. Brown, Heegner Modules and Elliptic Curves, Lecture Notes in Math. 1849, Springer, Berlin, 2004. MR 2082815. DOI 10.1007/b98488.

[2] B. H. Gross, "Kolyvagin's work on modular elliptic curves" in $L$-functions and Arithmetic (Durham, 1989), London Math. Soc. Lecture Note Ser. 153, Cambridge Univ. Press, Cambridge, 1991, 235-256. MR 1110395. DOI 10.1017/CBO9780511526053.009.

[3] B. H. Gross and D. Zagier, Heegner points and derivatives of L-series, Invent. Math. 84 (1986), 225-320. MR 0833192. DOI 10.1007/BF01388809.

[4] J.-I. Igusa, Fibre systems of jacobian varieties III (Fibre systems of elliptic curves), Amer. J. Math. 81 (1969), 453-476. MR 0104669.

[5] V. A. Kolyvagin, "Euler systems" in The Grothendieck Festschrift, II, Progr. Math. 87, Birkhäuser, Boston, 1990, 435-483. MR 1106906.

[6] Finiteness of $E(\mathbb{Q})$ and $\amalg(E / \mathbb{Q})$ for a subclass of Weil curves, Izv. Akad. Nauk SSSR Ser. Mat. 52, no. 3 (1988), 522-540, 670-671. MR 0954295.

[7] _ "On the structure of Shafarevich-Tate groups" in Algebraic Geometry (Chicago, IL, 1989), Lecture Notes in Math. 1479, Springer, Berlin, 1991, 94-121. MR 1181210. DOI 10.1007/BFb0086267.

[8] - On the structure of Selmer groups, Math. Ann. 291 (1991), 253-259. MR 1129365. DOI 10.1007/BF01445205.

[9] W. G. McCallum, "Kolyvagin's work on Shafarevich-Tate groups" in L-functions and Arithmetic (Durham, 1989), London Math. Soc. Lecture Note Ser. 153, Cambridge Univ. Press, Cambridge, 1991, 295-316. MR 1110398. DOI 10.1017/CBO9780511526053.012.

[10] J. S. Milne, Arithmetic Duality Theorems, Perspect. Math. 1, Academic Press, Boston, 1986. MR 0881804.

[11] M. Raynaud, "Caractéristique d'Euler-Poincaré d'un faisceau et cohomologie des variétés abéliennes" in Dix exposés sur la cohomologie des schémas, Adv. Stud. Pure Math. 3, North Holland, Amsterdam, 1967, 12-30. 
[12] J. P. Serre, Propriétés galoisiennes des points d'ordre fini des courbes elliptiques, Invent. Math. 15 (1972), 259-331. MR 0387283.

Institut Fourier, Boîte Postale 74, 38402 Saint Martin d'Hères, France; martin.brown@ujf-grenoble.fr 